\title{
REAL-TIME SENTIMENT-BASED ANOMALY DETECTION IN TWITTER DATA STREAMS
}

\author{
A Thesis \\ Submitted to the Faculty of Graduate Studies and Research \\ In Partial Fulfillment of the Requirements \\ FOR THE DEGREE OF \\ MASTER OF SCIENCE \\ IN \\ Computer Science \\ UNIVERSITY OF REGINA
}

\author{
By
}

Khantil Ragnesh Patel

Regina, Saskatchewan

March 31, 2016

Copytright (c) 2016: K.R. Patel 


\section{UNIVERSITY OF REGINA}

\section{FACULTY OF GRADUATE STUDIES AND RESEARCH SUPERVISORY AND EXAMINING COMMITTEE}

Khantil Ragnesh Patel, candidate for the degree of Master of Science in Computer Science, has presented a thesis titled, Real-Time Sentiment-Based Anomaly Detection in Twitter Data Streams, in an oral examination held on March 30, 2016. The following committee members have found the thesis acceptable in form and content, and that the candidate demonstrated satisfactory knowledge of the subject material.

External Examiner:

Co-Supervisor:

Co-Supervisor:

Committee Member:

Chair of Defense:

*Via Video Conference
*Dr. Nathalie Japkowicz, University of Ottawa

Dr. Howard Hamilton, Department of Computer Science

Dr. Orland Hoeber, Department of Computer Science

Dr. Robert Hilderman, Department of Computer Science
Dr. Douglas Farenick, Department of Mathematics and Statistics 


\section{Abstract}

Twitter has over 316 million active users and the engagement of these Twitter users results in the rapid production of data, notably in the context of popular topics (such as news stories, politics, and sports). This data is available in the form of data streams, which has led many researchers to develop analysis techniques especially for Twitter data streams. Although anomaly detection in time series is a well established research area, its application to detect sentiment-based anomalies in large volumes of streaming data began recently. A sentiment-based anomaly is defined as a sudden increase in the time series of tweets individually associated with a positive, neutral, or negative sentiment. The goal of this research is to develop and evaluate a technique to automatically detect sentiment-based anomalies, while avoiding the repeated detection of anomalies of similar types. Detecting anomalies in data streams is challenging due the requirement that anomalies be detected in real-time.

We propose an approach for real-time sentiment-based anomaly detection (RSAD) in Twitter data streams. Sentiment classification is used to split the input data stream into three independent streams (positive, neutral, and negative), which are then analyzed separately for anomalous spikes in the number of tweets. Rare anomalies and the first occurrence of repeated anomalies are distinguished from the repeated occurrence of similar anomalies. Six approaches for anomaly detection in data streams, including two baseline approaches, are described. These approaches were tested on two user-generated datasets. The first dataset concerned an international sports event and was collected from Twitter and the second concerned a political party and was collected from multiple social media platforms. Results from these evaluations show that a probabilistic exponentially weighted moving average (PEWMA), coupled with a sliding window that uses a median absolute deviation (MAD) calculation, is effective at identifying sentiment-based anomalies. The PEWMA-MAD approach is 
consistently among the top two methods for all cases tested. The simple linear regression approach is slightly better in the case of the second dataset. Overall, the results suggest that the PEWMA-MAD approach may be robust sufficiently to be applied to a wide variety of datasets from different social media platforms. 


\section{Acknowledgments}

I would like to thank my senior co-supervisor Dr. Howard Hamilton for his support and guidance throughout my years as a student. Under his supervision I had lot of opportunities to learn and grow my abilities to conduct the research that has real impact through the collaboration with the industry partners. I feel exceedingly appreciated to have had his guidance and I owe him a great many heartfelt thanks.

I would also like to thank my co-supervisor Dr. Orland Hoeber. His quality of giving attention to every detail at work has thought me to work with perfection. His ideas, suggestions and brainstorming sessions were very helpful throughout the process of developing and writing this thesis.

I am grateful to the Faculty of Graduate Studies and Research, the Department of Computer Science, Nature Sciences and Engineering Research Council of Canada, and of course again my supervisors, for their generous financial support during the course of my M.Sc. study.

I would also like to thank many people in our department who have helped me on many occasions over the years. I thank my friends for their help. A particular acknowledgement goes to the members of the visualization group including Maha El Meseery, Kenneth Odoh, Manali Gaikwad, and the members of the computer graphics group including Andrew Geiger, Daniel Lavin, Fatemeh Bayeh, and Stamatis Katsaganis. 


\section{Dedication}

I would like to dedicate this work to my parents, Ragnesh and Jagruti Patel. Without your support and encouragement, none of this would have been possible. I would like to thank my grandmother Pushpa Patel for the inspiration that I have received from her life, and the spiritual support during difficult times. I would also like to thank my brother Nehul Patel and sister-in-law Charmi Patel, for always being there, my friends (Birju, Dhanu, and Manas) for being the best friends anyone could ask for, and Manali Gaikwad for her support and encouragement. At last I would like to thank my dearest sister Nirali Patel and brother Shrey Patel for bringing out best in me. 


\section{Contents}

$\begin{array}{lll}\text { Chapter } 1 & \text { Introduction } & 1\end{array}$

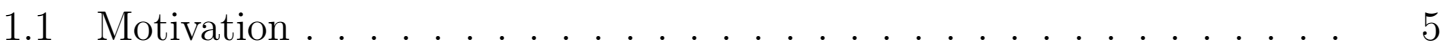

1.2 Problem Statement . . . . . . . . . . . . . . . . . . . . 8

1.3 Approch Overview . . . . . . . . . . . . . . . . . . . 10

1.4 Thesis Organization . . . . . . . . . . . . . . . . . . . . 11

$\begin{array}{lll}\text { Chapter 2 } & \text { Background and Related Work }\end{array}$

2.1 Data Stream Mining . . . . . . . . . . . . . . . . . 12

2.1.1 Data Stream Models . . . . . . . . . . . . . . . 13

2.1.2 Challenges in Data Stream Mining . . . . . . . . . . 15

2.2 Anomaly Detection in Time Series Data Streams . . . . . . . . . 16

2.2.1 Factors for Selecting an Anomaly Detection Technique . . . . 18

2.2.2 Overview of Anomaly Detection Techniques . . . . . . . . . 21

2.2.3 Extreme Value Analysis . . . . . . . . . . . . . . . 24

2.2.4 Simple Linear Regression . . . . . . . . . . . . . . . . . . . . 28

2.2.5 Local Outlier Factor . . . . . . . . . . . . . . . 31

2.3 Analysis of User-generated Content from Twitter . . . . . . . . . . 35

2.3.1 Time Series Analysis . . . . . . . . . . . . . . 36

2.3.2 Sentiment-Based Time Series Analysis . . . . . . . . . . . . . 39 
$\begin{array}{lll}\text { Chapter } 3 & \text { The RSAD Approach } & 42\end{array}$

3.1 Anomaly Formalization . . . . . . . . . . . . . . . . . . . . 42

3.2 Overview of the RSAD Approach $\ldots \ldots \ldots \ldots \ldots$

3.2.1 Step 1: Preprocessing . . . . . . . . . . . . . . . 46

3.2.2 Step 2: Two-stage Real-time Anomaly Detection . . . . . . . . 48

3.3 The Online TRAD Algorithm . . . . . . . . . . . . 56

3.4 Computational Complexity . . . . . . . . . . . . . . . . . . 59

3.5 Implementation in the Apache Storm Framework . . . . . . . . 60

3.5 .1 Concepts in Storm . . . . . . . . . . . . . . . . 61

3.5.2 Twitter Analytics Topology _... . . . . . . . . . . . 63

3.5.3 Integration of Continuous Input Twitter Streams _ . . . . . 64

3.5.4 Preprocessing the Stream . . . . . . . . . . . . . . 65

3.5.5 Real-time Anomaly Detection using TRAD . . . . . . . . . 67

$\begin{array}{lll}\text { Chapter } 4 & \text { Evaluation Methodology and Results }\end{array}$

4.1 Algorithms . . . . . . . . . . . . . . . . . . . . . . . . . 69

4.1.1 Two-stage Real-time Anomaly Detection . . . . . . . . . . 70

4.1.2 Simple Linear Regression Analysis . . . . . . . . . . . . . . 71

4.1 .3 Local Outlier Factor . . . . . . . . . . . . . . . 72

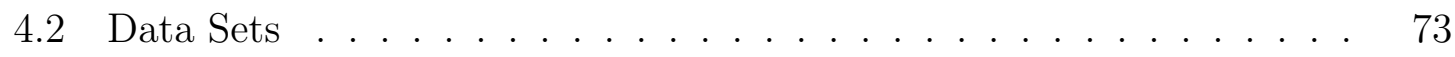

4.2.1 Le Tour de France 2013 Dataset . . . . . . . . . . . . . . . 73

4.2 .2 The Gavagai Dataset . . . . . . . . . . . . . . . 75

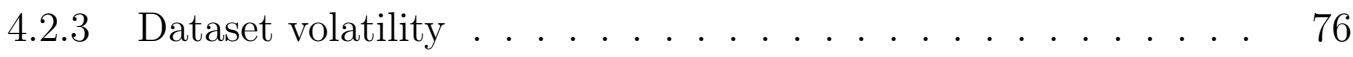

4.3 Experimental Procedures and Environment . . . . . . . . . 78

4.4 Results . . . . . . . . . . . . . . . . . . . . . . 79

4.4.1 Le Tour de France 2013 Dataset . . . . . . . . . . . . . . 80 
4.4 .2 The Gavagai Dataset . . . . . . . . . . . . . . . 92

4.4.3 Summary of Results . . . . . . . . . . . . . . . 103

$\begin{array}{lll}\text { Chapter } 5 & \text { Conclusions } & 107\end{array}$

5.1 Contributions ......................... 107

5.2 Limitations and Future Work . . . . . . . . . . . . . . . 110

$\begin{array}{ll}\text { References } & 114\end{array}$

Appendix A $\quad$ Detailed Results for TDF 2013 dataset 122

A.1 Evaluation for Window Size in TDF 2013 . . . . . . . . . . . . . . 122

Appendix B $\quad$ Detailed Results for Gavagai dataset $\quad 124$

B.1 Evaluation for Window Size in Gavagai dataset . . . . . . . . . . . 124 


\section{List of Tables}

3.1 Queries table used by the Storm topology . . . . . . . . . . . 65

4.1 Results for the EWMA-STD approach with the TDF dataset . . . . . 81

4.2 Results for the EWMA-MAD approach with the TDF dataset . . . . 82

4.3 Results for the PEWMA-STD approach with the TDF dataset . . . . 83

4.4 Results for the PEWMA-MAD approach with the TDF dataset . . . 84

4.5 Average F-score summary for TRAD with the TDF dataset . . . . . 87

4.6 Results for the SLR approach with the TDF dataset . . . . . . . . 88

4.7 Results for the LOF approach with the TDF dataset . . . . . . . . . 90

4.8 Average F-score summary for the TDF dataset . . . . . . . . . . . . . 91

4.9 Results for the EWMA-STD approach with the Gavagai dataset . . . 93

4.10 Results for the EWMA-MAD approach with the Gavagai dataset . . 94

4.11 Results for the PEWMA-STD approach with the Gavagai dataset . . 95

4.12 Results for the PEWMA-MAD approach with the Gavagai dataset . . 96

4.13 Average F-score summary for TRAD with the Gavagai dataset . . . . 99

4.14 Results for the SLR approach with the Gavagai dataset . . . . . . . . 100

4.15 Results for the LOF approach with the Gavagai dataset . . . . . . . . 102

4.16 Average F-score summary with the Gavagai dataset . . . . . . . . . . 102

A.1 Window size results for TRAD approach with the TDF Dataset . . . 123

A.2 Window size results for SLR approach with the TDF Dataset . . . 123 
A.3 Window size results for LOF approach with the TDF Dataset . . . 123

B.1 Window size results for TRAD approach with the Gavagai Dataset . 125

B.2 Window size results for SLR approach with the Gavagai Dataset . . . 125 


\section{List of Figures}

1.1 Example of Twitter and Facebook post for the query \#electioncanada 3

1.2 Tweets related to topic \# \#electioncanada . . . . . . . . . . . . . . . 6

2.1 An example of time series data . . . . . . . . . . . . . 21

2.2 Time series with mean and standard deviation . . . . . . . . . . 25

2.3 Applying a linear regression model to time series data. . . . . . . . . 30

2.4 Illustration of reachability distance in LOF . . . . . . . . . . . . . . . 32

2.5 Overview of LOF technique . . . . . . . . . . . . . . 34

2.6 Modeling time series as LOF, adapted from [52] . . . . . . . . . 35

3.1 Types of concept drift in time series data streams. . . . . . . . . . . . 44

3.2 Synthetic time series data streams, presenting the legitimate and candidate anomalies. . . . . . . . . . . . . . . . . 46

3.3 Overview of real-time sentiment-based anomaly detection . . . . . . . 47

3.4 Candidate anomaly detection stage: EWMA versus PEWMA . . . . . 53

3.5 Legitimate anomaly detection stage: STD versus MAD . . . . . . . 56

3.6 Core concepts in Apache storm, adapted from [4] . . . . . . . . . . 61

3.7 Storm topology for overall system . . . . . . . . . . . . . . 63

3.8 Internal working of the redesigned Storm spout . . . . . . . . . . . 64

3.9 Preprocess module with multistage bolts . . . . . . . . . . . . 66

3.10 Monitor module with multistage bolts for the TRAD approach . . . . 67 
4.1 The Le Tour de France 2013 dataset . . . . . . . . . . . . . . 74

4.2 The Gavagai dataset $\ldots \ldots \ldots \ldots \ldots \ldots$

4.3 F-score results for the EWMA-STD approach with the TDF dataset . 81

4.4 F-score results for the EWMA-MAD approach with the TDF dataset 82

4.5 F-score results for the PEWMA-STD approach with the TDF dataset 83

4.6 F-score results for the PEWMA-MAD approach with the TDF dataset 84

4.7 F-score results for the SLR approach with the TDF dataset . . . . . 88

4.8 F-score results for the LOF approach with the TDF dataset $\ldots . .90$

4.9 F-score results for the EWMA-STD approach with the Gavagai dataset 93

4.10 F-score results for the EWMA-MAD approach with the Gavagai dataset 94

4.11 F-score results for the PEWMA-STD approach with the Gavagai dataset 95

4.12 F-score results for the PEWMA-MAD approach with the Gavagai dataset 96

4.13 F-score results for the SLR approach with the Gavagai dataset . . . . 100

4.14 F-score results for the LOF approach with the Gavagai dataset . . . . 102

4.15 Results summary bar chart . . . . . . . . . . . . . . . . . . . 103

4.16 Results summary for the SLR technique . . . . . . . . . . . 105 


\section{Chapter 1}

\section{Introduction}

In recent years, social media has become an important source of information. User-generated content is commonly created in the form of text, images, and video and posted on social media platforms such as Facebook, Google+, LinkedIn, Tumbler, and Twitter. These platforms have revolutionized the way a user can generate and share information with individuals, groups, and communities. Users choose to use social media platforms as information sharing tools because of the unique communication services that they provide, such as portability, immediacy, and ease of use, which allows users to instantly respond to and spread information with limited or no restriction on content [21]. Users share timely and fine-grained information about many kinds of ongoing events, often reflecting their personal perspectives, emotional reactions, and controversial opinions. Virtually any person involved in or following an event is able to share information in real-time. This information can thus reach anywhere in the world as the event unfolds. For instance, in January 2011, during the political crisis in Egypt, citizens turned to Twitter to spread news around the world when the government blocked all news agencies [38]. Thus, social media may be considered a valuable source of up-to-date information generated by groups of users in the context of almost any event. 
The main sources of up-to-date information on social topics (e.g. elections, sports, and education) are social media and traditional sources, such as news channels, websites, or radio channels. The information published by the traditional sources covers only a few events, especially well planned ones, and does not provide extensive user reactions. Considering these limitations of traditional sources, social media platforms are the only resources available that are capable of providing real-time information on all but the most widely covered social topics. Content can be published on social media in real-time by users who are either attending an event or just interested in sharing their view about the event. This information contains the diverse opinion of thousands of social media users. The information generated from social media platforms may provide timely, actionable, and sometimes fact-based insights about social topics, which are not available in real-time through any other sources.

The user-generated information available on social media can be exploited to reveal insights into any social topic in real-time. For example, consider a political context, where an analyst, researcher, or other interested person wishes to stay informed about activities and updates related to an on-going federal election in Canada. The relevant user-generated data can be analyzed for more than basic news gathering. For example, they can be used to detect events (e.g. debates, speeches) or microevents (e.g. candidate announcements, controversies) and further, to recognize the sentiment of users on social media by analyzing their opinions and reactions. Perhaps most interestingly, election results may be predicted before voting by identifying the candidates towards which the maximum number of users have expressed a positive sentiment [48].

Facebook, Google+, and Twitter are the most popular text-based social media platforms. These platforms allow users to post information related to an event using a specific hash tag (e.g., \#electioncanada). An analyst can then search this information 
using the same hash tag and obtain a list of all posted information mentioning this hash tag, as shown in Figure 1.1. In the figure, the three most recent posts on the topic are visible; these topics are updated in real-time as new comments are posted (not shown). The analyst can read through the list to get a rough overview of what users are saying about the topic. However, this list of information is potentially

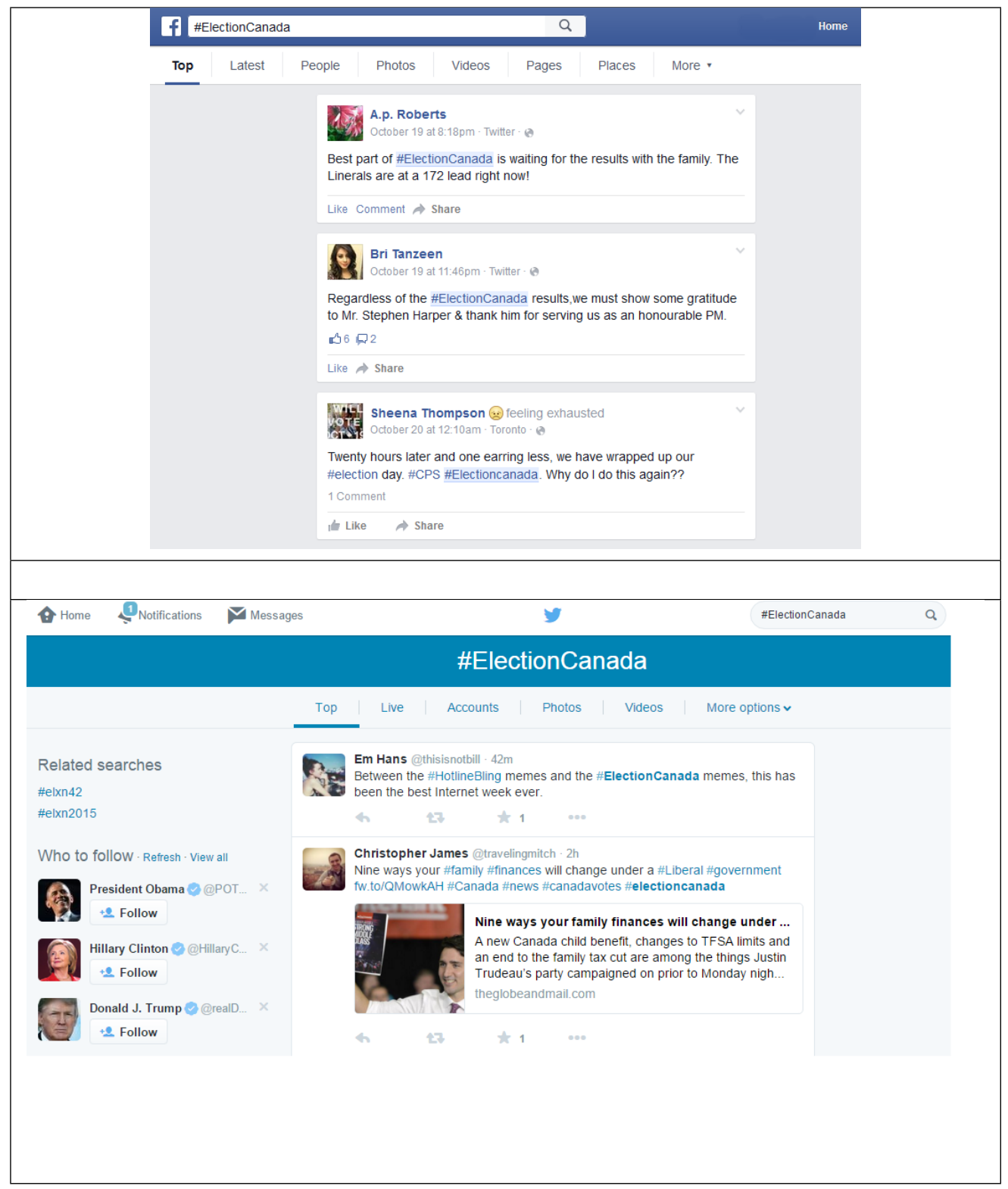

Figure 1.1: List of information posted by the social media users on Facebook (top) and Twitter (bottom) for the query \#electioncanada on 20 October 2015 
endless and may be updated at a rate greater than the analyst's cognitive ability to process the new information. Analyzing this information is so time consuming that it may prevent an analyst from making insightful analysis in order to answer questions, such as "What are the top five topics related to election being discussed?" and "Which candidate is the subject of the most postings with negative sentiment?".

In order to quickly answer such insightful questions, the abundance of information generated by social media can be turned into an opportunity, allowing the analyst to combine background knowledge with the computer's ability to store and process this information [31]. Many social media platforms have made user-generated content available for data analysis in the form of data streams. Data streams are a popular way of characterizing the voluminous and almost continuous flow of user-generated data [8]. A naïve approach to processing a data stream for knowledge extraction is to collect and store the data and then analyze the data using traditional data analysis methods, such as data mining and machine learning. This process can be automated by choosing to perform the data analysis periodically. However, analyzing the stored data off-line introduces some delay in the timeliness of the extracted knowledge and also consumes huge amounts of storage space.

There is an increasing need to develop scalable techniques for analyzing social media data streams in real-time. Employing real-time data stream analysis methods automates the data analysis process and provides the opportunity to extract meaningful insights in a timely manner. However, the task is more challenging than storing and then analyzing the data offline, because it poses strict constraints on the space and time available for computation [7,41]. Example applications of analysing social media data streams include opinion mining, sentiment analysis, detecting trending topics and events, and anomaly detection $[9,10,28,46]$. 
The remainder of this chapter is organized as follows: Section 1.1 states the motivation for work in this Thesis. Section 1.2 describes the problem to be addressed and formalizes the goals for the research. Section 1.3 gives an overview of the proposed RSAD approach. Section 1.4 provides the organization of the remaining chapters in the Thesis.

\subsection{Motivation}

The work in this thesis is focused specifically on user-generated content from the Twitter social media platform. Millions of Twitter users express their opinions on a wide range of topics on a daily basis, producing large amounts of data that is modelled as data streams and analyzed for valuable insights. Twitter has made such data streams publicly available for data mining purposes through their public streams service [50], in contrast to other social media platforms like Facebook or LinkedIn, where information is only accessible to people that are friends or connections of the person who posted the information. Assessing this service allows real-time collection of streams of tweets related to any specified topic keywords, hash tags (\#), or user names (@). This availability of public streams has enabled researchers to propose and study a broad range of techniques for analyzing Twitter data, including visual analytics [26, 27], sentiment analysis [8, 42], and anomaly detection [24].

An interesting fact about user engagement on Twitter is that the users tend to post their opinions in relation to specific events (e.g. sports, elections) and activities (e.g. shopping, adventures) in which they are explicitly involved or just interested in talking about. While doing so, they employ hash tags to annotate tweets with

the context of a specific topic, as well as other noteworthy aspects. In order to estimate the popularity of a topic on Twitter, a simple approach is to calculate the 
number of tweets posted (per minute or hour) using the topic's hash tag. Based on this approach, researchers have developed techniques for event detection through tweet frequency time series, assuming that a sudden peak in the number of tweets is an indicator of a micro-event that is taking place in the context of an observed topic $[5,16,34]$.

Detecting the peaks in the frequency of tweets reveals that a topic is becoming popular due to users actively tweeting about it. In order to gain insight into the actual cause of the increased popularity of a topic, one currently has to personally examine the tweets during that period. The tweet frequency time series may contain hidden trends that can be uncovered by decomposing the time series into several component series, each corresponding to a different sentiment [46]. Analysing these decomposed sentiment series (i.e., positive, negative, and neutral) instead of just the frequency of tweets is useful because it gives independent information about users' different opinions. The utility of this decomposition is demonstrated in Figure 1.2,

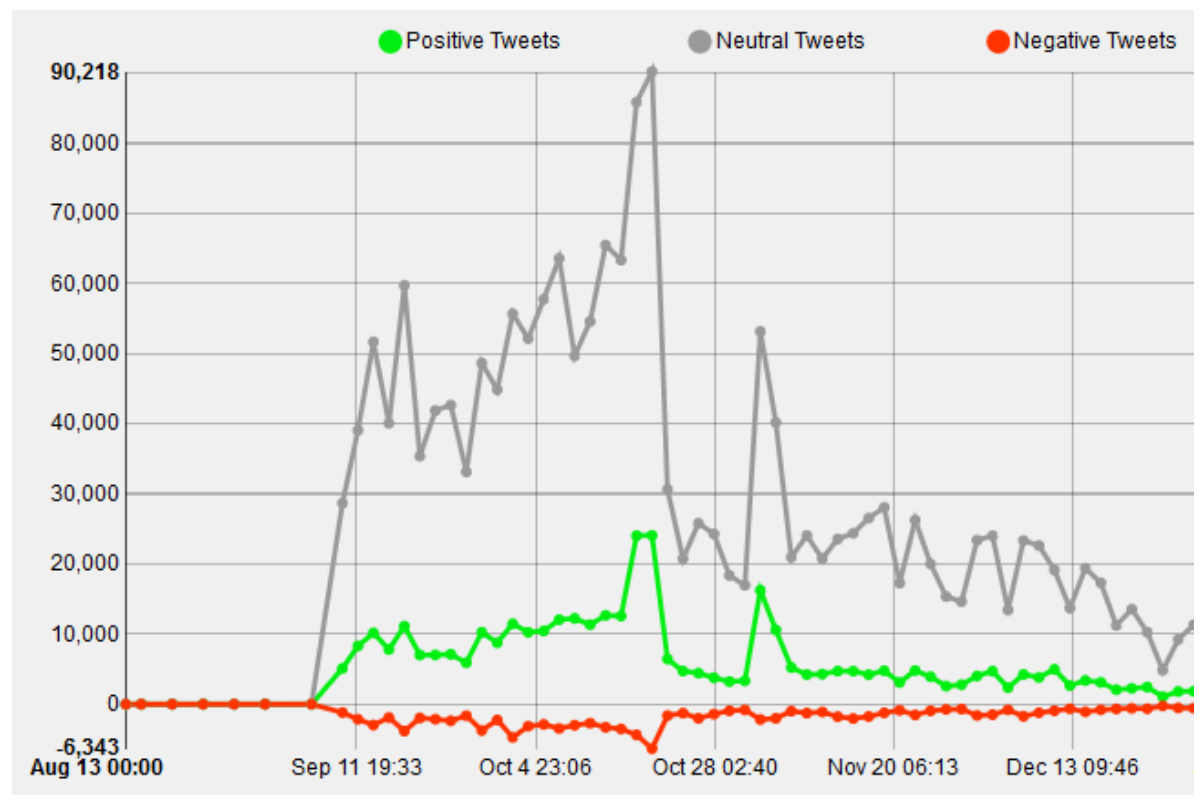

Figure 1.2: Tweets related to topic \#electioncanada with (2 day) aggregated sentiment tweets, from 13 April 2015 to 7 August 2015 (original in colour) 
which shows a time series plot representing the variation in the popularity of the topic "\#electioncanada". The three time series shown in the figure corresponds to the positive (green), negative (red) and neutral (grey) sentiments, as obtained by applying sentiment analysis. The peaks at September 18th, October 20th, and November 3rd, depict sudden increases in the popularity of the topic in correlation with sudden changes in both positive and negative sentiment. The peak at October 20th is due to negative or somewhat mixed reactions from the users, while the peak at November 3rd is mostly due to positive reactions from users.

Mining tweets based on their sentiments to uncover the reason behind the popularity of a topic is more effective than just using the frequency of tweets. When sentiment classification is performed over the tweets associated with a specific topic's hash tag, it can help discover a more nuanced description of the public perception of that particular topic by opinion [42]. The primary motivation for this work is derived from the fact that sudden increases in the number of tweets tagged with a specific topic are often the result of strong sentiment expressed in the tweets by the users [46]. The use of strong sentiment influences a large numbers of users to react, producing bursts of tweets. Over time it may become difficult to understand the sentiment for the topic of interest in such a large amount of text. In such a scenario, detecting a sudden bias of the users towards a specific sentiment as an anomaly can reveal an overall shift in the users' opinions related to that topic.

In the remainder of this Thesis, a sentiment-based anomaly is defined as a sudden increase in the volume of tweets individually associated with a positive, neutral, or negative sentiment. The timely detection of such sentiment-based anomalies will enable data analysts associated with businesses, government, or sport management to intervene in response to positive reaction or negative reaction. 


\subsection{Problem Statement}

The work in this thesis addresses the problem of providing an analyst with timely information about opinions relevant to topics of interest without requiring continual observation. Visual analytics approaches have been used to discover and analyze the temporally changing sentiment of tweets posted in response to micro-events occurring during a multi-day sporting event $[26,27]$. However, in order to discover noteworthy micro-events in real-time that cause unexpected increases (or spikes) in the number of positive, neutral, or negative tweets, the analyst must monitor the system as events occur. Such monitoring would be time consuming and thus not cost effective in many situations.

The goal of this research is to automatically detect, in real-time, sentiment-based anomalies in Twitter data streams. Such sentiment-based anomalies can be passed to analysts as alerts to conduct further analysis immediately and perhaps take action. The intention is to detect a change in the number of tweets in each sentiment class independently (e.g., increases in the positive tweets) even if they are masked by an inverse change in another class (e.g., decreases in the negative tweets).

Since the data streams generated from Twitter are a nearly continuous and unbounded sequences of tweets ordered by their timestamps, the three sentiment classified data streams are also ordered by their timestamps. Hence, it is appropriate to cast each as a time series data stream. Anomaly detection in such a stream is difficult for two main reasons. First, the dynamic nature of the data stream may result in changes in the data distribution over time, which is called concept drift [41]. For example, the distribution of tweets using a specific hashtag on one day may be different from the distribution on another day because of the occurrence of an event that resulted in a change in the use of this hash tag. Secondly, since the data streams 
may be considered to be infinite series, storing and analyzing all of the data points is not feasible. Thus, given the desire to detect anomalies in real-time, the anomaly detection technique should use models and data structures that can be incrementally updated and adhere to space and time efficiency constraints [2]. The major assumptions for the research presented in this thesis are:

1. the data stream is generated from a normal distribution with mean $\mu_{t}$ and standard deviation $\sigma_{t}$;

2. an anomaly is defined with respect to a sliding window of a given length;

3. anomaly detection is performed for a user specified topic given in advance;

4. anomaly detection is performed independently for each positive, negative, and neutral classes of sentiment.

The goals for the research presented in this thesis are listed below:

1. Formalize a definition for sentiment-based anomaly such that it will allow the analyst to independently detect rare anomalies in each class of sentiment on Twitter with respect to a sliding window of a given length.

2. Develop a technique to detect the sentiment-based anomaly such that,

(a) It detects sentiment-based anomalies in near real-time on a high-velocity data stream with a fixed amount of storage and satisfies the run time complexity constraint.

(b) The technique should be resilient to temporal concept drift.

3. Implement the real-time sentiment-based anomaly detection (RSAD) technique in the context of Twitter,

(a) The implementation should be able to processes the Twitter data stream and execute the proposed sentiment-based anomaly detection technique (Goal 2) in real-time.

(b) The implementation should be robust and scalable, such that the anomaly 
detection can be concurrently conducted with respect to more than one topic.

4. Evaluate the sentiment-based anomaly detection technique proposed in Goal 2 and implemented in Goal 3, and perform comparative analysis with baseline anomaly detection techniques.

\subsection{Approch Overview}

In order to address these problems and goals, a real-time sentiment-based anomaly detection (RSAD) approach is proposed. It operates in two main steps: pre-processing and anomaly detection. In the pre-processing step, tweets in a data stream are classified using a sentiment classifier and then accumulated in bins of a fixed user-specified time interval (e.g., 15 minutes). The resulting binned values are treated as data points in the time series. The anomaly detection step uses two-stage real-time anomaly detection (TRAD). First a candidate anomaly is detected by identifying a significant difference between the current data point and the distribution of recent data points. Secondly, the candidate anomaly is compared to other previously detected candidate anomalies stored within a sliding window of a fixed user-specified length (e.g., five days). If this candidate anomaly deviates sufficiently from those in the sliding window, it is considered a legitimate anomaly. When a legitimate anomaly is detected, an alert can be sent to an analyst. The alert indicates that the analyst may wish to inspect recent tweets from this data stream to discover the reason for a change in the pattern of the number of tweets that are being posted with a specific sentiment. The parameters for the binning time interval (aggregation interval) and the length of the sliding window (window length) are specified by the analyst based on domain specific knowledge about the characteristics of the data stream with respect to the 
topics under investigation.

\subsection{Thesis Organization}

The remainder of this thesis is organized as follows. In Chapter 2, an introduction to data stream models and data stream mining is given. Background concepts and challenges related to anomaly detection in time series data stream are discussed. Further, a review of the core techniques for detecting anomalies in time series data streams is provided. A brief review of work related to time series analysis is given, with emphasis on sentiment-based time series analysis in the context of user-generated content.

In Chapter 3, a formal definition of an anomaly in the context of this thesis is defined. An overview of the proposed RSAD approach is given, followed by a detailed discussion of the two-stage real-time anomaly detection approach (TRAD). This work proposes the TRAD approach for which an online algorithm is given, along with its scalable implementation in the Apache Storm Framework.

Chapter 4 describes the experiments performed to compare the proposed TRAD approach with two alternative approaches. Two real world datasets that were generated from user-generated content are described along with their characteristics. The results from the experiments performed for each candidate approach and dataset are presented, along with a discussion on the findings.

Finally, Chapter 5 provides a brief review of the work accomplished in this thesis and a comparison to the goals stated in this Chapter. Moreover, a discussion describing the limitations of the proposed RSAD approach is presented along with the future research work that can be conducted in order to overcome the limitations and develop additional features. 


\section{Chapter 2}

\section{Background and Related Work}

This chapter provides background information concerning three aspects of this research. The first of these is data stream models, as described in Section 2.1. The second is the core techniques for anomaly detection in time series data, which are described in Section 2.2. Section 2.3 gives a literature review on time series analysis and its application to user-generated content from Twitter.

\subsection{Data Stream Mining}

Mining data from streams is challenging because traditional data mining techniques cannot be readily applied to data streams [23]. To mine a data stream requires an algorithm that can analyze the data sufficiently quickly for real-time applications. Moreover, the memory consumption of the algorithm should also be restricted so as to maintain sufficient free memory to store newly arriving data. The rest of this section provides an overview of data stream modelling and the challenges of mining data streams. 


\subsubsection{Data Stream Models}

In recent years, various applications have emerged in which data is modelled as data streams. A data stream is continuous, rapidly moving data produced by applications such as financial systems, network monitoring, security, telecommunications, web applications, manufacturing, and sensor networks [2]. Formally, a data stream can be defined as [37]:

Definition 2.1.1 (Data Stream). A data stream is a sequence of datum $d_{1}, d_{2}, \ldots$ that arrive sequentially, item by item, and describes an underlying signal $A$, where in the simplest case $A$ is a one-dimensional function $A:[0 \ldots(N-1)] \rightarrow Z$.

A data stream can describe the underlying signal in various ways, resulting in a number of data stream models. The elements in a data stream may occur once or several times, and they may appear in a predefined order or in an unordered fashion. These characteristics of the elements describe the nature of the underlying signal. The three widely used data models are the Time Series, Cash Register, and Turnstile models [37].

With a Time Series type of model, data points give values in a time series. Thus, each $A[i]$ value equals the corresponding data point $d_{i}$, i.e. $A[i]=d_{i}$. This model is a well suited to time series data, such as the number of clicks per minute on a website or the number of tweets per minute on a topic. Consider the sequence 10,1,8,5, which serves as an example of a Time Series data stream. After the stream has been processed, the model $A$ is given as $A=[10,1,8,5]$, where the $i$ th entry in the vector denotes the value of $i$ th element.

With a Cash Register type of model, each data point $d_{i}=\left(j, I_{i}\right)$, where $I_{i} \geq 0$, gives an increment to $A[j]$. Let $A_{i}$ be the state of the signal after seeing the $i$ th item

in the stream. To process $d_{i}, A$ is incremented as $A_{i}[j]=A_{(i-1)}[j]+I_{i}$. Consider the 
sequence $(2,7),(1,4),(2,3),(4,5)$ which serves as an example of a Cash Register data stream. Assuming an initial model of $[0,0,0,0,0, \ldots, 0]$, the final model A after the stream has been processed is given as $A_{4}=[4,10,0,5,0, \ldots, 0]$, where the $j$ th entry in the vector denotes the frequency of occurrence of the $j$ th element.

With the Turnstile type of model, each data point $d_{i}=\left(j, U_{i}\right)$, where $U_{i}$ may be positive or negative, gives an update to $A[j]$. To process $d_{i}, A$ is updated as $A_{i}[j]=A_{(i-1)}[j]+U_{i}$. Suppose, that the sequence is $(2,7),(1,4),(2,-3),(4,-5)$. This sequence implies that the value at index 2 is increased by 7 , the value at index 1 is increased by 4 , the value at index 2 is decreased by 3, and so on. Assuming an initial model of $[0,0,0,0,0, \ldots, 0]$, the final model after applying these updates is given as $A_{4}=[4,4,0,-5,0, \ldots, 0]$, where $j$ th entry in the vector denotes the updated value of the $j$ th element.

The selection of an appropriate model for a data stream depends upon the characteristics of data in the stream as well as the type of analysis that needs to be performed. In this work, the data stream considered is a Twitter data stream in which the data points are tweet objects with explicit timestamps, i.e., $d_{i}=(i, T)$, where $i$ is the timestamp and $T$ is the tweet object. The tweet object is a string encoded in JSON format, which defines several properties associated with a tweet, such as tweet ID, text, timestamp, list of hash tags, geolocation, etc [50]. As mentioned in Section 1.2, the objective of the work in this thesis is to perform time series analysis and detection of anomalies in a Twitter data stream. Thus, based on the input data and the required analysis, the time series data stream model is selected as the most appropriate data stream model for our problem. 


\subsubsection{Challenges in Data Stream Mining}

Data Stream Mining is defined as the process of extracting knowledge structures from a data stream in near real-time using a restricted amount of memory space. Algorithms for data stream mining should be optimized for minimum time and space consumption. Considering this definition of data stream mining, traditional data mining techniques, which assume data is available for random access from a database or a file system, and analysis can be performed off-line, are not applicable.

The concept of data stream mining can be explained with an example based on anomaly detection in a sensor monitoring application. Suppose the data stream consists of the sensor readings that were generated every second by a group of sensors in a manufacturing facility and we have collected one month of these sensor readings. The size of the data is approximately 1 terabyte. The problem is to detect when system failures occurred during that month. A solution using a traditional data mining technique seems easy: collect the sensor data in a database or file system and then analyze this data by applying an off-line anomaly detection technique, which might take some minutes or hours to locate the system failures.

However, if the off-line technique is applied directly to the sensor data stream in an effort to detect system failures in near real-time, it would have difficulty. Initially it would try to store the streamed data locally or in-memory, and analyze it. Because analysis of the data requires time, the fixed-size local storage would quickly be filled by the stream of data. Further, the algorithm would cause increasing delays in processing and eventually stop executing due to lack of available memory. Clearly, traditional data mining techniques need to be adapted or replaced by new techniques in order to analyze data streams efficiently.

Analyzing data streams differs from the traditional stored data models in several ways, which can be viewed as three constraints that are imposed on data stream 
mining techniques [7]:

1. A data stream is potentially infinite in size, and thus it is impossible to store all the data points in storage of a limited size.

2. The need for near real-time output forces data points to be processed at approximately the rate that new ones are generated.

3. The underlying data distribution generating the data points can change over time. Thus, data from the past may become irrelevant or even harmful for the current analysis.

Constraint 1 limits the amount of memory that can be utilized. Therefore, only small summaries of the data stream needs to be extracted and stored at any given time, while the majority of the data points themselves can be discarded. Constraint 2 limits the time available to process each data point. These two constraints have led to the development of summarization techniques such as sliding window averages and aggregation. Constraint 3 requires the data mining algorithm to implement a forgetting mechanism, such that only recent summaries of the data are maintained in order to cope with changes in the data distribution.

\subsection{Anomaly Detection in Time Series Data Streams}

A time series encodes state information about a system along with a temporal factor. When considered from the perspective of anomaly detection, the temporal factor enriches this information, because it can help reveal time-critical insights. For example, a stream of clicks generated from an online shopping website could reveal an anomaly at a particular time, indicating a product that is receiving an unusually large number of clicks by the visitors of website. This information can be used by the owners to make an immediate decision to restock the product earlier than otherwise. 
The application of anomaly detection to time series data streams has been well studied by researchers in the data mining community [23]. Researchers have proposed anomaly detection techniques for a wide range of application domains, such as sensor monitoring [25], website load monitoring [29], cloud analytics [51], social media topic detection [24], and traffic monitoring [36].

While the earliest work in this field was proposed a decade ago [6], it remains an active field of research. Recently, a technique to detect anomalous work loads in cloud servers was proposed [29]; this technique is being used to detect increases in traffic as early as possible to prevent crashes and to perform load balancing. Anomaly detection in data streams is being studied extensively because it addresses the problem of monitoring critical application data for unusual activity, which is otherwise done by humans and may be prone to error. Anomaly detection can form an important part of automatic monitoring solution, which may be more reliable and accurate than human monitoring.

In the remainder of this section, several factors relevant to selecting an appropriate anomaly detection technique for the targeted application domain are first presented in Section 2.2.1. Then, an overview of the three core categories of the anomaly detection techniques, which are based on probabilistic and statistics models, prediction based models, and proximity based models, are given in Section 2.2.2. Each approach is categorized based on (a) its data model and (b) its approach for defining and detecting outliers. The Extreme Value Analysis, Simple Linear Regression and Local Outlier Factor approaches, which are representative of these categories, are presented in Sections 2.2.3, 2.2.4, and 2.2.5, respectively. 


\subsubsection{Factors for Selecting an Anomaly Detection Technique}

Diverse techniques have been proposed in the literature to address the problem of anomaly detection in time series data [23]. Several factors influence the choice of a specific technique. We present five general factors that will help to address questions that are often asked in order to clearly evaluate the requirements and the expectations for anomaly detection. These factors, which are to be considered at the initial stage of selecting an anomaly detection approach [3], are presented below.

The first factor is the data type. The data type of a time series data stream can be univariate or multivariate. Some applications, such as sensor monitoring and website statistics, generate univariate data in the form of numeric or text time series data. When the data is univariate, anomaly detection can be performed directly. Other applications, such as social media analytics and network monitoring, produce multivariate data (including JSON and XML objects) as time series. When the data is multivariate, preprocessing is often necessary to transform it to a univariate representation. Data transformation techniques such as Principle Component Analysis (PCA) and Symbolic Aggregate Approximation (SAX) can be applied to transform multivariate data into a univariate time series [35, 45].

The second factor is the data length. The length of the input data to the anomaly detection technique affects the accuracy of detecting anomalies. To be effective, most anomaly detection techniques require the length of the input data to be large. If the length is too short, techniques such as regression [6] may not give useful results. However, in such cases, robust statistic methods, such as the median [33] and tvalue analysis[3], can be adapted for use with existing techniques. If the length is acceptable (such that most techniques could be applied with acceptable accuracy and perform the analysis efficiently within the space and time complexity bounds), then no optimization is needed. If the data length is infinite, such as occurs with data 
streams, then the technique should address the data stream analysis constraints, as discussed in Section 2.1.2.

The third factor is the data label. A label is a boolean value associated with a data point in a training sample that indicates whether the instance is normal (false) or anomalous (true). Obtaining labelled data is difficult, because the labeling typically needs to be done by a human expert who has comprehensive domain knowledge. However, even if labeled data is obtained, it may happen that some types of anomalies are not present in a training dataset. Based on the extent to which labeled data is available, anomaly detection techniques can operate in the following three modes [14]. In the supervised anomaly detection mode, labeled data are available with both normal and anomalous labels. In such a scenario, a probabilistic or predictive classification model can be built with normal and anomalous classes. Any unseen data are then compared against the model to determine if they belong to the normal class or the anomalous class. In the semi-supervised anomaly detection mode, it is assumed that all training data points are implicitly tagged with normal labels. The techniques operating in this mode do not require labelled data for training, because they can model the similarity in the data as normal and categorize any peculiarities as anomalies. Such techniques are suitable for streaming data because they only learn a model of the normal classes and they can readily update this model as new data arrives. In the unsupervised anomaly detection mode, it is assumed that labelled data is not available for training. However, these techniques make a general implicit assumption that normal data points are placed closely to one another, whereas anomalies are located distantly from other data points. Furthermore, the normal data points appears far more frequent, whereas anomalies are rare. If this assumption is not true, then such techniques suffer from a high false positive rate.

The fourth factor is the interpretability of the model. Interpretability of the 
anomaly detection model is important from the analyst's perspective. When the anomalies are visible in the data, they can be interpreted. However when the anomalies are hidden in the data, the data need to be transformed and analyzed in a different space. Different models have different levels of interpretability. If a transformation or decomposition of a time series is performed that helps to expose anomalies, it may nonetheless lose the context of the anomaly. To improve interpretability one has to choose a model that does not transform the data such that it becomes difficult to match to the original data. For example, in the field of visual analytics [30], a 2D visualization of the data may be prepared. An analyst can diagnose the causes of detected anomalies by exploring and interacting with this visualization to gain a better understanding of them. When an anomaly is detected, one can intuitively understand why it is an anomaly in the context of the remaining data if the visualization is welldesigned. This intuitive understanding can help the analyst perform more detailed research in a domain specific scenario.

The final factor is the output format of the anomaly detection technique. The output format is related to the level of interpretability needed to gain insight into the cause of an anomaly. The output of an anomaly detection technique can be either outlier scores or binary labels [14]. An outlier score is a numeric value determined by evaluating the quality of fit between the data point and the normal model. Typically larger scores indicate more anomalous data. An outlier score provides all the information produced by algorithm, but it does not indicate which specific outliers are anomalous. Outlier scores are useful as output when the model provides a low level of interpretability. In contrast to an outlier score, a binary label simply tells whether or not a data point is an anomaly. Some algorithms may directly return binary labels. However, outlier scores can be converted into binary labels by imposing thresholds on outlier scores based on statistical distribution, for example by performing extreme 
value analysis. Binary labels are useful as output when the anomaly detection model provides a high level of interpretability. However, when the model provides a low level of interpretability, a binary label may contain less information than needed for decision making in real applications.

\subsubsection{Overview of Anomaly Detection Techniques}

In this section, a general approach for detecting anomalies is presented and three categories of anomaly detection techniques are briefly described. Consider the example time series data shown in Figure 2.1, which is referred to throughout this section.

In general, depending on the type of output, an anomaly detection process consisting of the following steps:

1. From the raw input data, generate a data model that is suitable for further analysis.

2. Compute an outlier score or binary label for each data point in the data model by evaluating the quality of fit between the data point and the normal data using the detection technique.

3. If the output is an outlier score but is needed as a binary label, then check if the outlier score of a data point is greater than a threshold and if so, output the data

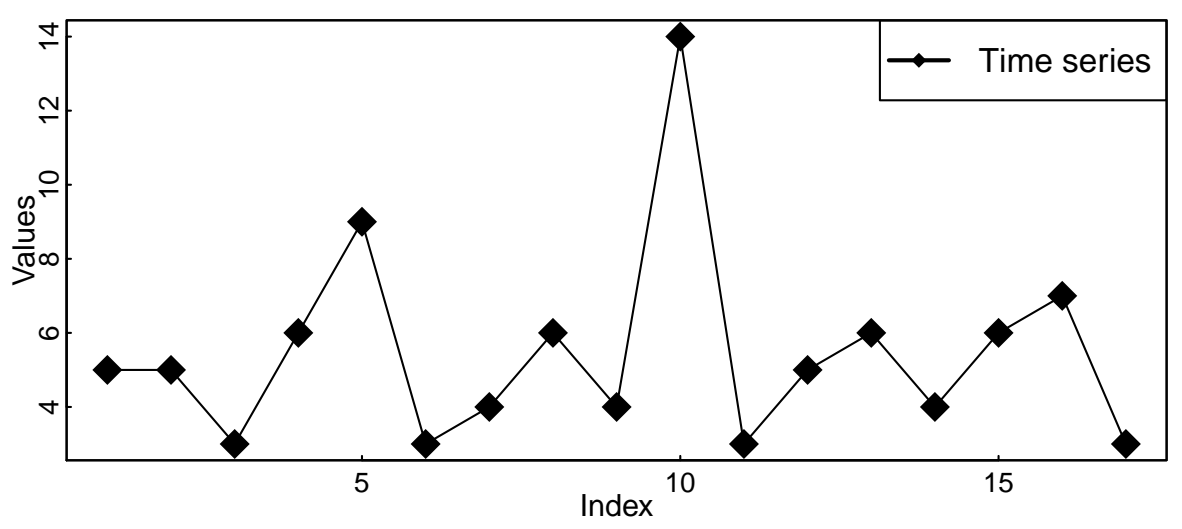

Figure 2.1: An example of time series data 
point with a true binary label; otherwise, output the data point with a false binary label.

Anomaly detection techniques are categorized based on the ways in which Step 1 and Step 2 are performed (i.e, how the data is modelled and which approach is used for defining and detecting outliers. Although a wide range of techniques have been proposed in the literature to calculate an anomaly score, many techniques for univariate time series data streams can be placed in one of three core categories. The core categories are based on the type of model users during analysis. The three core categories are probability distribution models, prediction based models, and proximity based models.

Probability Distribution Models: A probability distribution model is learned from the training data set or learned dynamically from the input data stream. Then the anomaly score for a given data point is calculated in terms of its probability of being generated from the learned model, with a higher score indicating a higher possibility of being an outlier. Probability distribution model based techniques are placed in two groups based on the distribution model that is used to model the data. First, extreme value analysis (EVA) techniques, such as the $z$-value test [13] and the extreme studentized deviate test (ESD) [51], try to fit the data to a specific data distribution model, such as the Gaussian distribution, in order to produce an anomaly score. The parameters of these models can be estimated using the maximum likelihood method, which uses the standard deviation as the error of the mean. Second, mixture model based techniques, such as the kernel mixture [40, 54] and the Gaussian mixture [53] methods, use a mixture of data distributions (instead of a specific distribution) to generate the anomaly score. The parameters of mixture models may be learned using the Expectation-Maximization (EM) method.

Prediction Based Models: Commonly a prediction based model is a regression 
model; in such models, a data point is modelled using a system of linear equations [3]. A linear model is learned from the history of data points by estimating the regression coefficients. Then the model is used to predict the value of the next data point. The deviation between the predicted data point and the observed data point is called the prediction error, which may be used as an anomaly score. Higher prediction errors indicate a higher possibility of the data point being an outlier. As the model evolves, a regression line is gradually drawn using the current linear model. This line indicates the trends in the time series. Regression based models are popular for time series anomaly detection [6], but because they are computationally expensive, there is limited work in the literature that uses this technique for data stream applications.

Proximity Based Models: A proximity-based technique defines a data point as an outlier if its proximity (or locality) is sparsely populated. The proximity of a data point can be defined in two ways, distance-based and density-based. In a distancebased technique, greater distances to the neighbours of a targeted data point indicate increased chance of that point being an outlier. For example, with the k-nearest neighbour $(\mathrm{k}-\mathrm{NN})$ technique [11], the distance to the kth nearest neighbor is used. A higher distance to the kth nearest neighbour indicates a greater possibility of being an outlier. Distance-based techniques are used to detect global outliers, where a global outlier is an outlier with respect to all data in a time series. Finding global outliers is computationally expensive, but some optimizations, such as the use of indexing, have been proposed in order to adapt the $\mathrm{k}-\mathrm{NN}$ method to the context of a data stream [19].

In a density-based technique, the number of data points within a specified local region of a targeted data point is used to define proximity [11]. A lower number of data points in the local region of the targeted data point indicates a higher chance of the point being an outlier. Density-based techniques, such as the local outlier 
factor (LOF), are used to detect local outliers, which are outliers with respect to neighbouring data points in the time series. For the application to data streams, an optimized technique called incremental local outlier detection has been proposed [39].

\subsubsection{Extreme Value Analysis}

Extreme value analysis (EVA) is a simple statistical anomaly detection technique for univariate data [3]. As its name implies, this technique is capable of detecting specific kinds of outliers that are extremely large or small compared to the whole data set. Two well-known techniques for EVA are the $z$-value test and the modified $z$-value test.

\section{Z-value test}

The $z$-value test is a simple method for outlier analysis [3]. A implicit assumption is made that the data is generated from a normal distribution. The method learns and dynamically updates two parameters, the mean $(\mu)$ and the standard deviation $(\sigma)$, from the history of data points. Consider a series of univariate data points denoted by $d_{1}, \ldots, d_{t}$, with mean $\mu_{t}$ and standard deviation (STD) $\sigma_{t}$ at time $t$. The $z$-value for the data point $d_{t}$ is denoted by $Z_{t}$ and is defined as follows:

$$
Z_{t}=\frac{\left|d_{t}-\mu_{t-1}\right|}{\sigma_{t-1}}
$$

The $z$-value test computes the number of standard deviations by which data point $d_{t}$ varies from the mean at time $t$. The parameters $\mu_{t}$ and $\sigma_{t}$ model the parameters of the normal distribution of the data. In general, the density function $f\left(d_{t}\right)$ for a 
normal distribution with mean $\mu$ and standard deviation $\sigma$ is defined as follows:

$$
f\left(d_{t}\right)=\frac{1}{\sigma \cdot \sqrt{2 \cdot \pi}} \cdot \exp \left(\frac{-\left(d_{t}-\mu\right)^{2}}{2 \cdot \sigma^{2}}\right)
$$

A standard normal distribution is one in which the mean $\mu$ is 0 , and the standard deviation $\sigma$ is 1 . In cases where the mean and standard deviation of the input data distribution can be accurately modelled, it is a standard practice to consider $d_{t}$ as an anomaly if $Z_{t} \geqslant 3$ [36]. Figure 2.2 shows the mean and one standard deviation above and below the mean, for a time series example.

However, in many scenarios, the mean and standard deviation cannot be accurately calculated. First, if the sample size $t$ is too small then the model will overfit the data and result in false negatives $[15,43]$. In such cases, other variant methods which are robust for smaller sample sizes can be used. Two such methods are Grubb's test and the t-value test [3]. Second, if the sample size $t$ is infinitely large, then the mean and standard deviation can not be evaluated efficiently. In general, the sample

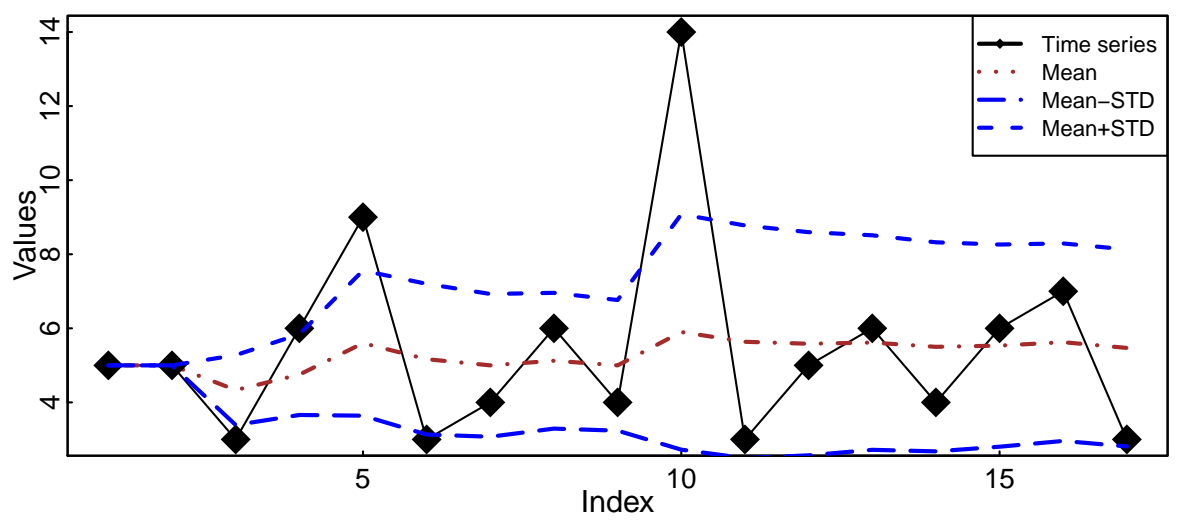

Figure 2.2: Time series example with mean and standard deviation learned from normal distribution mode 
mean and standard deviation is calculated as follows:

$$
\begin{gathered}
\mu_{t}=\frac{1}{t} \sum_{i=1}^{t} d_{i} \\
\sigma_{t}=\sqrt{\frac{1}{t-1} \sum_{i=1}^{t}\left(d_{i}-\mu_{(i-1)}\right)^{2}}
\end{gathered}
$$

where $i$ denotes the instance number of the current data point. As Equation 2.4 calculates the sample standard deviation, it is divided by $t-1$ instead of $t$. Here, if $t$ is large then it will take $O\left(t^{2}\right)$ time to update the mean and standard deviation for each new data point. There is a well known method of determining both mean and standard deviation with a single loop to achieve $O(t)$ time, and this method can be adapted to estimate $\mu_{t}$ and $\sigma_{t}[22]$.

$$
\begin{gathered}
\mu_{t}=\mu_{(t-1)}+\frac{d_{t}-\mu_{(t-1)}}{t} \\
\sigma_{t}=\sigma_{(t-1)}+\left(d_{t}-\mu_{(t-1)}\right) \cdot\left(d_{t}-\mu_{t}\right)
\end{gathered}
$$

As an alternative, the mean and standard deviation can be calculated using exponential methods such as the exponential weighted moving average technique (EWMA) [36]. EWMA computes $\mu_{t}$ and $\sigma_{t}$ of a time series by applying exponentially decreasing weight factors to each prior data point. If $t \leq k$, it uses Equations 2.3 and 2.4 for the initialization of $\mu_{t}$ and $\sigma_{t}$, where $\mathrm{k}$ is the number of training instances and else when $t \leq k$, it uses update equations:

$$
\begin{gathered}
\mu_{t}=\alpha \cdot \mu_{(t-1)}+(1-\alpha) \cdot d_{t} \\
\sigma_{t}=\alpha \cdot \sigma_{(t-1)}+(1-\alpha) \cdot\left|d_{t}-\mu_{(t-1)}\right|
\end{gathered}
$$


where $0 \leq \alpha \leq 1$ specifies the amount of weight to put on historical values in comparison to the most recent data point. One advantage of EWMA is that the computation process is simple, requiring few variables and little time. According to Equations 2.7 and 2.8, EWMA only requires the most recent values of $\mu$ and $\sigma$, i.e., their values at time $t-1$. EWMA is efficient for online analysis of large data streams and it has been widely used by researchers in the context of data stream anomaly detection $[13,36]$.

\section{The modified Z-value test}

The two parameters used in the Z-value test, the mean and standard deviation, can be highly affected by a few extreme values or even by a single extreme value [43]. To avoid this problem, the two parameters in the Z-value test can be replaced by the median and the median absolute deviation (MAD). The median is another measure besides the mean of the central tendency of an underling distribution of time series

data. It offers the advantage over the mean of being insensitive to the presence of extreme values. The test for detecting an outlier using the median is given as below:

$$
Z_{t(M A D)}=\frac{\left|d_{t}-M_{(t-1)}\right|}{M A D_{(t-1)}}
$$

where $M_{t}$ is the median at time $t$ and $M A D_{t}$ is the median absolute deviation at time $t$. This test is referred to as the modified $Z$-value test [3].

First, we show the calculation of the median $M_{t}$ by considering the time series 1 , $10,3,8,6,10,1000,3$. After sorting in ascending order, the values are $1,3,3,6,8$, 10, 10, 1000. We assume the data points are indexed sequentially from 1 to 8 . The average rank can be calculated as $(n+1) / 2$, which is 4.5 in our example. Therefore $M_{t}$ is the average of 6 and 8 , which is 7 . Once we have the median, calculating MAD is straightforward because we only need to find the median of the absolute deviations 
between the values and median $M_{t}$. We use an $M$ operator to indicate the median of a series of values, analogously to how the $\sum$ operator indicates summation. We use the equation given below:

$$
M A D_{t}=b \cdot M_{i=1}^{t}\left(\left|d_{i}-M_{t}\right|\right)
$$

where $d_{i}$ is a data point from the $t$ original observations and $M_{t}$ is the median of the $t$. Usually, $b=1.4826$, is a constant linked to the assumption of normality of data [33]. Continuing our example, we can now calculate the series of absolute deviations from the median as $(1-7),(3-7),(3-7),(6-7),(8-7),(10-7),(10-7),(1000-7)$, that is $6,4,4,1,1,3,3,993$. After this series is sorted, we obtain 1,1,3,3,4,4,6,993 and the median of these values is the average of 3 and 4 , which is 3.5 . We multiply the median by 1.4826 to calculate $M A D_{t}$ as 5.18. According to the test in Equation 2.9, all values greater than $7+(3 \times 5.18)=22.57$ and all values less than $7-(3 \times 5.18)=-8.57$ can be declared to be extreme value outliers. Recall that in the case of the $z$-value test using the mean, the accuracy is highly affected by the sample size. In contrast, the accuracy of MAD does not depend on the sample size and thus generally has fewer false negative results [33].

\subsubsection{Simple Linear Regression}

Simple linear regression is an approach to modelling the relationship between a dependent variable $Y$ and one or more independent variables $X[6]$. It helps to understand the characteristics of the dependent variable $Y$, for different values of $X$. Linear regression based analysis can be used to detect anomalies. The idea is to predict a forthcoming data point with a model that looks at the history of the data

points and then compare the predicted data point $\widehat{Y}$ with the real observed data point 
$Y$ as it arrives [52]. If the model fits the data well, the predicted value will be the same or close to the observed data point. However, it may happen that the observed data point deviates somewhat from the predicted data point. The deviation is called the residual or (prediction error) of the model. A linear model can be given as:

$$
\widehat{Y}_{t}=X_{t} \cdot \beta+\epsilon_{t-1}
$$

The output $\widehat{Y}_{t}$ is called a regressand or dependent variable. The parameter $X_{t}$ is called a regressor or independent variable. $\beta$ is the tunable parameter called the regression coefficient. $\epsilon_{t-1}$ is the prediction error of the previous prediction.

Assuming that the prediction model perfectly fits the data, the value of the prediction error will be zero, and we can make the model determinate by simply ignoring $\epsilon_{t-1}$,

$$
\widehat{Y}_{t}=X_{t} \cdot \beta
$$

The error for each predicted data point can be obtained as,

$$
\epsilon_{t}=Y_{t}-\widehat{Y}_{t}=Y_{t}-\left(X_{t} \cdot \beta\right)
$$

Figure 2.3 illustrates the regression line generated from a linear model estimated using Equation 2.12 for the example data. In this model, estimating the value of $\beta$ is a crucial step, because its accuracy will determine the fit of the model to underlying data distribution. Ordinary least squares (OLS) [3] is a method for calculating the unknown parameter $\beta$ in a the linear regression model. The goal of estimating $\beta$ is to determine a value, such that it minimizes the deviation between the observed values and the corresponding predicted values. If the deviation is small, the model 
fits better with the underlying data distribution. According to OLS, the estimation parameter $\beta_{t}$ is given as:

$$
\beta_{t}=\frac{t \cdot S_{x y}-S_{x} \cdot S_{y}}{t \cdot S_{x x}-S_{x}^{2}}
$$

where $S_{x y}=\sum_{i=1}^{t} X_{t} \cdot Y_{t}, S_{x}=\sum_{i=1}^{t} X_{t}, S_{x x}=\sum_{i=1}^{t} X_{t}^{2}$ and $S_{y}=\sum_{i=1}^{t} Y_{t}$.

The value of $\beta$ is evaluated for each data point and Equation 2.12 can be used to calculate the prediction for the value of the next data point. Further, the error or residual is calculated by comparing the predicted value and original value using Equation 2.13. As the error is calculated for each prediction, the history information for the errors $\epsilon_{1}, \ldots, \epsilon_{t}$ is maintained. We assume the errors have an approximately normal distribution. Thus, a density distribution function (Equation 2.2) of errors can be calculated using Equation 2.3 for the mean $\mu_{\epsilon_{t}}$ and Equation 2.4 for the standard deviation $\sigma_{\epsilon_{t}}$ of the errors.

With the linear regression approach, a data point $Y_{t}$ is considered anomalous if it deviates from the corresponding predicted data point $\widehat{Y}_{t}$. To detect such cases, a $Z$-value test can be used on the history of the prediction error parameter $\epsilon_{t}$. Thus, a data point having prediction error $\epsilon_{t}$ between $\left[\mu_{\epsilon_{t}} \pm 3 \cdot \sigma_{t(r)}\right]$ is considered to be

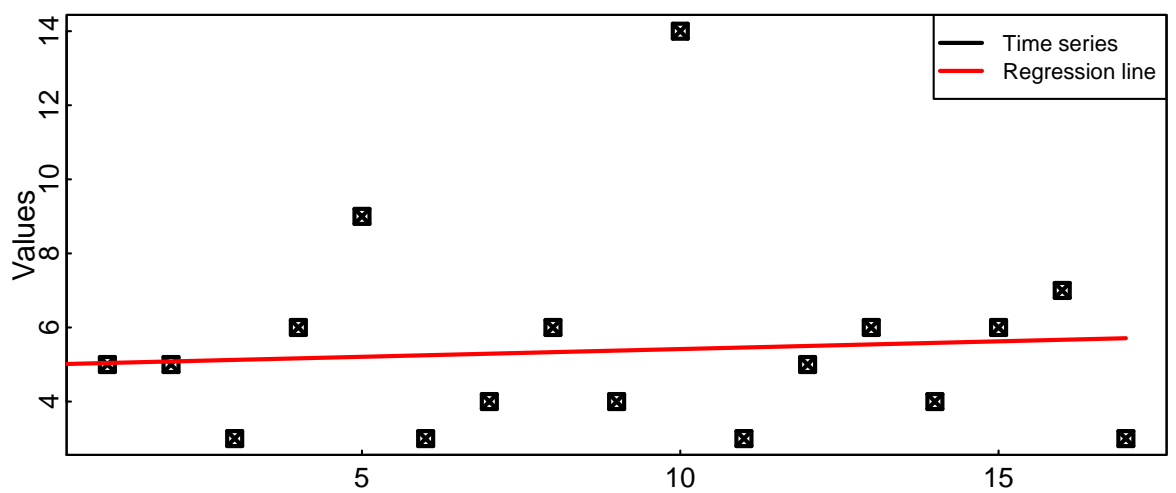

Figure 2.3: Applying a linear regression model to time series data. 
normal, and all others are considered anomalous. When creating a regression based model of a time series, the $X$ component of the model is the timestamp. Ordinarily the timestamp is replaced by an integer, which is incremented by a constant amount between data points, as shown in Figure 2.3.

\subsubsection{Local Outlier Factor}

The local outlier factor (LOF) technique [11] is a density based method that detects outliers relative to their local neighbourhoods, particularly with respect to the density of their neighbourhoods. The method builds on the k-NN technique, which is applied to determine the denseness of the neighbourhood of a data point in comparison to that of neighbouring data points. Every data point is given an individual LOF score reflecting how densely its neighbourhood is populated compared to others. Data points with LOF scores higher than some threshold are labeled as anomalous.

\section{Definition of LOF}

LOF was initially proposed in the context of multivariate data. First, the LOF technique is explained with reference to a 2-dimensional model. Then its applicability to time series data is explained. Consider a data point $d$ that belongs to dataset D. The following concepts and definitions [11] are needed to understand the LOF algorithm:

Definition 2.2.1 ( $k$-distance of data point $d[11]$ ). For any positive integer $k$, the $k$ distance of an object $d$, denoted as $k$-distance $(d)$, is defined as the Euclidian distance $\operatorname{dist}(d, o)$ between $d$ and an data point $o \in D$ such that:

- for at least $k$ data points $o^{\prime} \in D \backslash\{d\}$ it holds that $\operatorname{dist}\left(d, o^{\prime}\right) \leq \operatorname{dist}(d, o)$ and

- for at most $k-1$ data points $o^{\prime} \in D \backslash\{d\}$ it holds that $\operatorname{dist}\left(d, o^{\prime}\right)<\operatorname{dist}(d, o)$ 
As shown in Figure 2.4 considering $k=3$, the 3 -distance of datapoint $d$ is given as $\operatorname{dist}\left(d, o_{3}\right)$, such that for at least $3(k)$ data points in $D \backslash\{d\}$ i.e. $\left\{o_{1}, o_{2}, o_{3}\right\}$, it holds that $\operatorname{dist}\left(d,\left\{o_{1}, o_{2}, o_{3}\right\}\right) \leq \operatorname{dist}\left(d, o_{3}\right)$. Moreover, for at most $2(k-1)$ data points in $D \backslash\{d\}$ i.e. $\left\{o_{1}, o_{2}\right\}$, it holds that $\operatorname{dist}\left(d,\left\{o_{1}, o_{2}\right\}\right)<\operatorname{dist}\left(d, o_{3}\right)$.

Definition 2.2.2 ( $k$-distance neighbourhood of an data point $d[11]$ ). Given the $k$ distance of $d$, the $k$-distance neighbourhood of $d$ contains every data point whose distance from $d$ is not greater than the $k$-distance, i.e.

$$
N_{k}(d)=\left\{o^{\prime} \in D \backslash\{d\} \mid \operatorname{dist}\left(d, o^{\prime}\right) \leq k \text {-distance }(d)\right\}
$$

The data points in $N_{k}(d)$ are called $k$-nearest neighbours of $d$. Note that the size of $N_{k}$ does not necessarily always equal $k$, but at all times it is at least $k$. The inequality occurs when more than one $k$-distance data point is at the same distance from $d$,

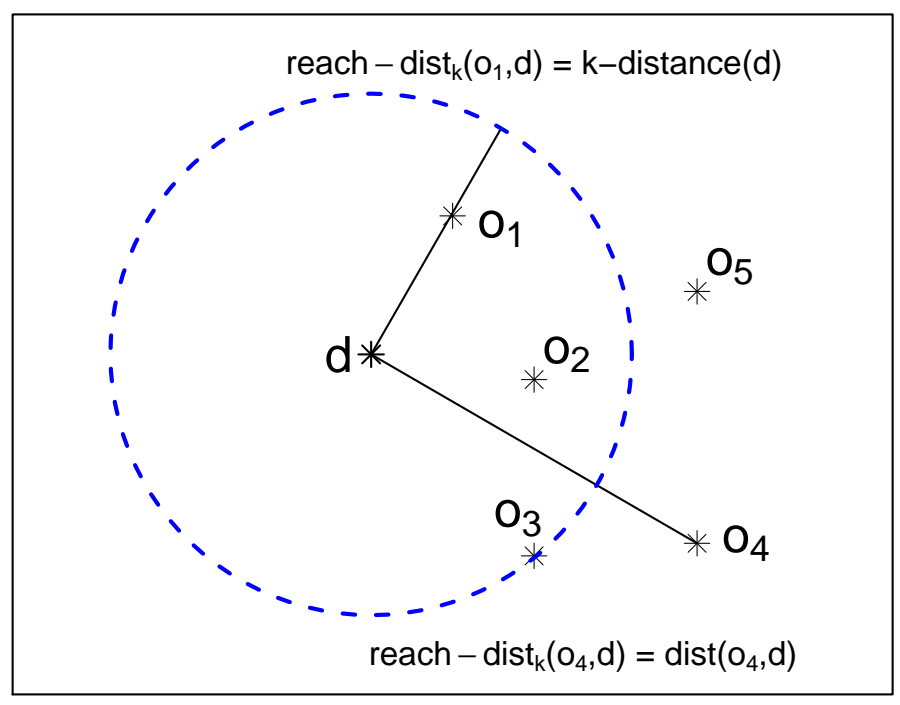

Figure 2.4: Reachability distance when $k=3$. Since $o_{1}$ is a k-nearest neighbour, its reachability distance is its $\mathrm{k}$-distance. In contrast, the reachability distance of data point $o_{4}$ is its true distance since it is not a k-nearest neighbor. 
i.e., more than one data point is on the circumference. As shown in Figure 2.4, the set of data points in the 3-distance $(d)$ neighbourhood of data point $d$ is given as $N_{3}(d)=\left\{o_{1}, o_{2}, o_{3}\right\}$. If $o_{5}$ were at the same distance as $o_{3}$, then the 3 -distance neighbourhood of data point $d$ would be $N_{3}(d)=\left\{o_{1}, o_{2}, o_{3}, o_{4}\right\}$.

Definition 2.2.3 (Reachability distance of a data point $d$ w.r.t $o$ [11]). The reachability distance of an data point $d$ with respect to data point $o$ is defined as maximum of the two distances $k$-distance $(o)$ and $\operatorname{dist}(d, o)$

$$
\text { reach-dist }{ }_{k}(d, o)=\max \{k \text {-distance }(o), \operatorname{dist}(d, o)\}
$$

Figure 2.4 illustrates the definition of reachability distance with $k=3$. The reachability distance of $o_{4}$ with respect to data point $d$ is $\operatorname{dist}\left(d, o_{4}\right)$, while the reachability distance of $o_{1}$ with respect to the data point $d$ is $k$-distance $(d)$. Now, that we have a concept of how to measure the distance between two data points, the local reachability density function of a data point $d$ can be explained:

Definition 2.2.4 (Local reachability density [11]). The local reachability density (LRD) of a data point $d$ is defined as the ratio between number of $k$-nearest neighbours $\left|N_{k}(d)\right|$ and the sum of the reachability distances, with respect to $d$, for these k-nearest neighbours:

$$
L R D_{k}(d)=\frac{\left|N_{k}(d)\right|}{\sum_{o \in N_{k}(d)} \text { reach-dist }{ }_{k}(d, o)}
$$

LRD of a data point indicates how densely populated its neighbourhood area is. Now, the final step in the algorithm is to determine the LOF score of each observation.

Definition 2.2.5 (Local outlier factor [11]). The local outlier factor (LOF) score of 


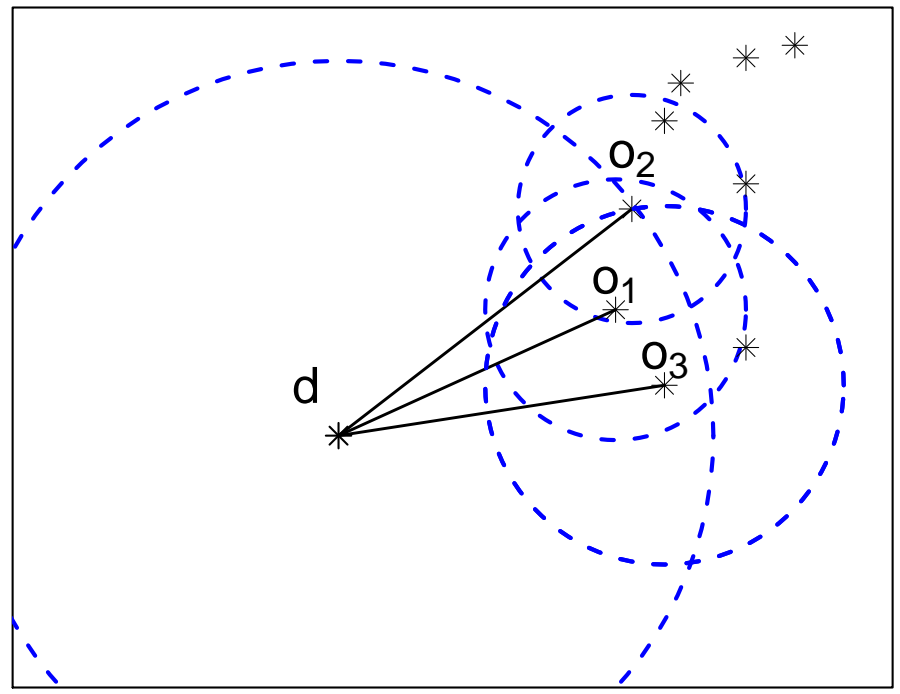

Figure 2.5: Illustrate the LOF algorithm with $k=3$, The data points in right corner are far more densely populated than the observation $d$. This will lead $d$ to get higher LOF score

a data point $d$ is defined as:

$$
L O F_{k}(d)=\frac{\sum_{o \in N_{k}(d)} \frac{L R D_{k}(o)}{L R D_{k}(d)}}{\left|N_{k}(d)\right|}
$$

The LOF score captures the degree to which $d$ is acting like an outlier. The LOF score is high whenever the neighbourhood density of $d$ greatly deviates from its neighbouring data points. Figure 2.5 shows an example where $L O F_{k}(d)$ is expected to have a high value, because its neighbourhood is not as densely populated as those of its neighbours. In contrast, the LOF scores for $o_{1}, o_{2}$, and $o_{3}$ are expected to have low values. 


\section{Applying LOF to Time Series Data}

To apply the LOF technique to detect anomalies in time series data, the time series can be transformed to a one-dimensional frequency plot, as shown in Figure 2.6. The distance $\operatorname{dist}(d, o)$ between two data points, $d$ and $o$, in a time series is defined as the absolute value of the difference between the two data points:

$$
\operatorname{dist}(d, o)=|\operatorname{value}(d)-\operatorname{value}(o)|
$$

The absolute value of the difference is used, because the distance should be nonnegative. To detect anomalies, we model a normal distribution function around LOF scores, by evaluating the mean $\left(\mu_{L O F}\right)$ and the standard deviation $\left(\sigma_{L O F}\right)$ of the LOF scores for each data point. Then using the $Z$-value test, we classify any data point whose LOF score is not between $\left[\mu_{L O F} \pm 3 \cdot \sigma_{L O F}\right]$ as anomalous.

\subsection{Analysis of User-generated Content from Twitter}

Recall that the work in this thesis is focused on user-generated content from the Twitter social media platform. A literature review of time series analysis in the
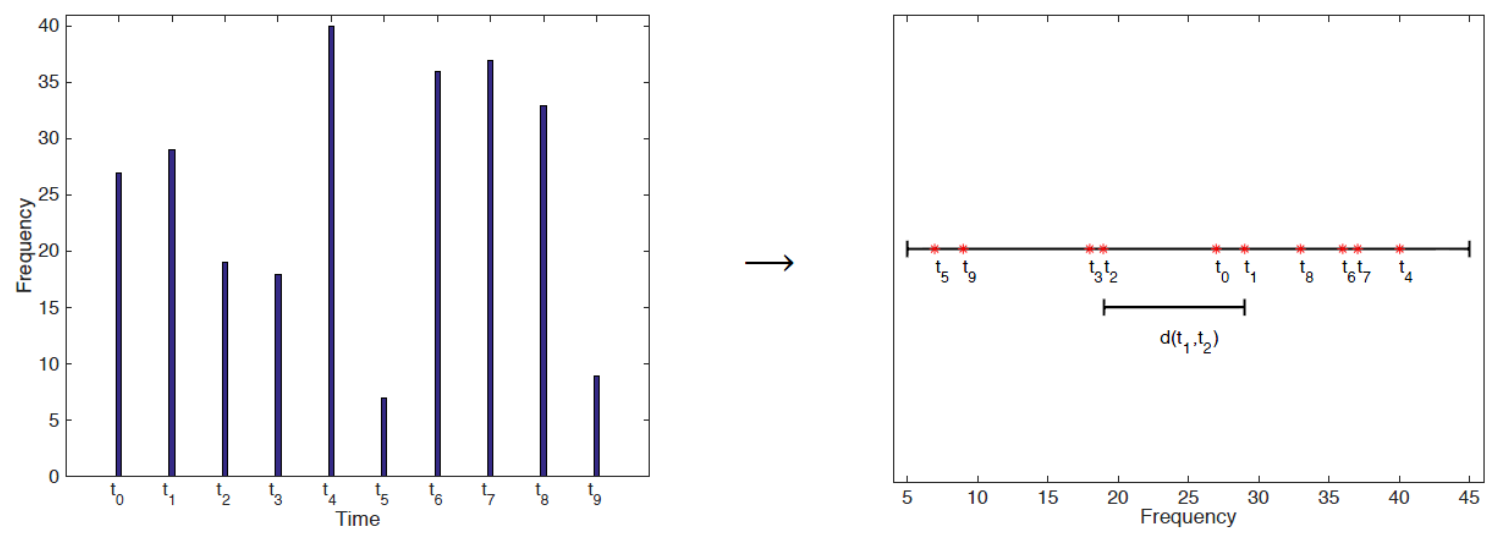

Figure 2.6: Modeling time series as LOF, adapted from [52] 
context of Twitter data is provided in Section 2.3.1. The application of time series analysis to Twitter data is also described. The Section 2.3.2 gives a review of previous work related to the application to Twitter data of one specific time series analysis technique, which is sentiment-based time series analysis.

\subsubsection{Time Series Analysis}

The number of tweets posted on Twitter in relation to a topic tends to change with time. One reason these changes occur is that users who are interested in the topic tend to post tweets during or immediately before or after an event related to that topic. Here a topic refers to a name representing a real world event that is being discussed on Twitter (such as a Canadian football game \#CFL or a federal election \#election), while a micro-event (or subevent) refers to a small event that occurs in the context of the main event (for example, in a Canadian football game, a touchdown is a micro-event and in a federal election, a debate is a micro-event).

Several previous studies $[5,16,34]$ have analyzed the tweeting patterns of users in response to an event from a time series perspective. When modelling the social media data as a time series, the general approach is to aggregate all data relevant to selected topics based on a fixed time interval (seconds or hours) to generate a regular time series. In the resulting time series, the peaks and lows become apparent, revealing the evolution of interest in the topics over time.

We assume that sudden changes in the tweet frequency are mostly due to the occurrence of events that influence enough users on Twitter to post tweets. Researchers have proposed and applied existing data mining techniques for identifying such changes in order to detect events on Twitter. Marcus et al. developed Twit-

Info [34], a tool that provides a visualization of a timeline of tweets containing a queried topic, which updates in real-time. The temporal peaks in tweet frequency 
are highlighted by an automated event detection algorithm which uses an exponential weighted moving average to detect peak time intervals where the frequency exceeds a given threshold. The text of the relevant tweets in such an interval is further analysed to identify the top keywords that describe the underlying micro-event. A case study on the tweets related to the topic of a soccer game illustrated that while the algorithm was able to detect most of the micro-event during the soccer game, it also produced a few false negatives for which there was no spike in the timeline. The results imply that only those micro-events were detected for which users choose to engage on Twitter. Thus, the reliability of the algorithm depends on the Twitter users interest in a micro-event.

Avvenuti et al. developed an earthquake alert and report system (EARS) [5], which is able to identify earthquake events in real-time by applying a detection algorithm on the set of tweets related to earthquake topics on Twitter. It automatically broadcasts the detected events via Twitter and email notifications. A pre-filtering phase removes tweets that use the earthquake related keywords with different meanings or that refer to past events. In order to identify large scale and small scale events, the algorithm tests the tweet frequency per small-duration window against a threshold. Further, the threshold is dynamically updated depending on the tweet frequency that is calculated per long-duration window. When the small-duration and longduration window values were set to 1 minute and 1 week, respectively, their system detected the occurrence of most earthquakes with magnitude greater than or equal to 3.5 from Twitter, within seconds of the actual event. They reported an F-score of 0.85. The detected events were posted far earlier than the official notification issued by the National Institute of Geophysics and Volcanology. The use of small-duration and long-duration windows increased the efficiency of EARS for real-time analysis, but the algorithm is sensitive to the selected window lengths and thus required tuning 
of those parameters by an expert with domain knowledge. The rate of false positive was increased by nonsense tweets from fake accounts and by problem with language detection while filtering the data in the initial stage.

Culatta [16] proposed a method for predicting rates of influenza-like illness in a population by analyzing tweets related to a few influenza related keywords. The method employs both simple and multiple linear regression models, which are initially trained with the labelled influenza statistics data provided by the U.S government. The trained regression models are then used to predict the true proportion of the population exhibiting influenza symptoms. Culatta determined the accuracy of the predictions by comparing them with the labelled influenza statistics data that was published in weekly reports and concluded that the multiple linear regression outperforms simple linear regression model because the former showed a higher correlation with the true statistics. The overall residual between estimated and true data was considered too high for this approach to be put to practical use. Moreover, the regression based model is costly in terms of memory and computation, and thus may be not feasible for real-time analysis.

The studies reviewed here demonstrate that time series analysis of frequency of tweets is a promising research direction. Overall, the event detection work in the literature has been performed with one of two main intentions. The first intention is to detect an unexpected event such as catastrophic disasters, financial crisis, and terrorist attack as soon as it happens, based on sudden changes in the tweet frequency. The second intention is to predict a future event based on the recent tweets, assuming a strong correlation exist between a trending topic with an exponentially increasing number of tweets on Twitter and a real event such as the spread of a disease, an election, or a riot. The event detection approaches show promising results with acceptable accuracy, but it is difficult to deduce the cause of such events by simply 
analyzing the frequency of tweets.

\subsubsection{Sentiment-Based Time Series Analysis}

Recent studies $[9,46]$ have shown a strong relation between real events and emotions expressed by users on Twitter. In order to identify a trend associated with the users' emotions as expressed in tweet text, Bollen et al.[9] applied a sentiment analysis approach that measures the mood in six dimensions: tension, depression, anger, vigour, fatigue, and confusion. Further, an off-line time series analysis based on the $z$-score and variance normalization was performed individually on the trend line for each mood dimension to highlight the peak periods. The experiments revealed that real world events related to social, political, and economic topics are correlated with significant abrupt changes in the trend lines of individual mood dimensions. These results suggest that the occurrence of such events later influences the users to express their reactions with strong sentiment in the tweets. Moreover, Thewall et

al.[46] studied these hypothesis from the opposite direction, i.e., whether the peaks of events triggered by large reactions on Twitter are always associated with an increases in the strength of expressed sentiment. The SentiStrength algorithm was used to deduce the overall sentiment score for a time interval. Several such scores were then aggregated to produce a time series for each topic under assessment. The authors concluded that the overall sentiment level was quite low. Increases in negative sentiment had a significant impact on the main peaks in Twitter, but the level of positive sentiment has limited impact.

Considering the evidence described above that abrupt changes in sentiment can have a high correlation with the peak in tweets on Twitter, many researchers have attempted to enhance sentiment analysis methods and to demonstrate the need for analyzing aggregated Twitter sentiment for the detection of interesting events by 
looking at anomalous peaks in sentiment. The detection of such interesting events that are influenced by sentiment would be difficult through the analysis of time series data consisting of general tweet frequency, because the change in one class of sentiment could be masked by a complementary change in another class, resulting in no change in the overall frequency of tweets.

Wang et al.[56] proposed an enhanced sentiment analysis method based on lexiconbased classifiers that is specifically designed for the analysis of tweet text with emoticons and special lexicon handling. They demonstrated the effectiveness and usefulness of the proposed enhancements by showing their applicability to anomaly detection through sentiment analysis on tweets collected related to a service provided by a company whose name was not disclosed. They performed an off-line manual analysis by graphing the frequencies of three different sentiment patterns. Their analysis was based on the affective events theory which claims that people's emotional responses are influenced by events that shape their attitudes and behavior. The results were consistent with the theory, in that during one period the graph showed significant increase in negative tweets, and this period was later matched with a controversial news item regarding the same service.

Another study presented by Diakopoulos et al.[18] considered the tweets related to the U.S Presidential debate in 2008 and showed a correlation between the peaks in the positive and negative sentiments on the topics of financial recovery and terrorist threats in the debate. The task for sentiment classification of tweets was outsourced to Amazon Mechanical Turk (AMT), a crowd-sourcing site where workers complete short tasks for small amounts of money.

Several many similar research efforts focused on improving sentiment analysis techniques $[10,28]$. Both efforts included an application that demonstrated the individual analysis of the aggregated Twitter sentiment classes to detect the anomalous 
peaks in an off-line manner. These efforts emphasize the importance of accurate sentiment analysis and its direct impact on the efficacy of specific applications that use sentiment analysis as a preprocessing step.

While the work reviewed in this section does provide a motive and partial solution, to the best of our knowledge no existing work in the literature focuses specifically on developing or applying existing automatic time series analysis techniques for detecting real-time anomalies in Twitter streams for separate sentiment classes. 


\section{Chapter 3}

\section{The RSAD Approach}

In this chapter, a real-time sentiment-based anomaly detection (RSAD) approach for detecting sentiment-based anomalies is presented. Section 3.1 presents a formal definition of an anomaly in the context of our research. Section 3.2 presents the RSAD approach, in which the Twitter data stream for a specific user-specified query is preprocessed, after which a two-stage real-time anomaly detection (TRAD) is performed. An online algorithm for the TRAD approach is given in Section 3.3 and the computational complexity of this algorithm is discussed in Section 3.4. In Section 3.5, we describe a scalable implementation of the RSAD approach in the Apache Storm framework which allows simultaneous analysis of tweets related to multiple queries.

\subsection{Anomaly Formalization}

The definition of the term anomaly with respect to a time series is often given in a vague manner because it depends on the specific anomalous behavior in the data that is of interest to the analyst, which is often based on the application domain [41]. Anomalies in different domains are different in nature and from each other. For example, because network traffic is bursty, only exceptionally large bursts will be considered to be anomalies; in contrast, since remote sensor networks commonly 
measure smooth and continuous phenomena, a small burst may be considered an anomaly in this setting.

The primary domain of the work in this Thesis is the user-generated content from Twitter. Twitter provides this data in the form of data streams. From the data modeling perspective, data streams have a unique characteristic in which the data distribution generating the data points has a tendency to change over time. When this change in underlying distribution appears in the data it is referred to as temporal evolution, non stationarity, or temporal concept drift [12]. Concept drift occurs due to the unpredicted substitution of one data source with another source. In the context of the Twitter data stream, data is generated when the users post the tweets related to a topic. Thus, the users are the data sources who generate the data in the Twitter data streams. Concept drift appears in the Twitter data stream when the tweeting pattern of the users or the number of actively tweeting users changes.

The tweets in the Twitter data stream arrive sequentially at irregular time interval. Given such a temporally irregular series of tweets, the task of detecting concept drift becomes challenging. In order to analyze the change in the temporal pattern of number of tweets, regular time series of tweets should be constructed. This can be achieved by aggregating the tweets over consecutive time intervals of predetermined length (e.g., 5 minutes). The process of grouping a number of continuous tweets within a time interval into a bin value $\left(d_{t}\right)$ is called temporal binning, and the length of time interval is called the temporal bin length. The result of temporal binning is a temporally regular series of bins, which is a time series data stream of tweets.

Concept drift may appear in the time series data stream in different forms over time. When concept drift occurs, the mean of the data changes. Depending on the various rates at which concept drift occurs, there are two main types of changes that may appear in a single variable along time as defined in the literature [12]: sudden 
and gradual. In sudden concept drift (depicted in Figure 3.1(a)), the changes that instantly and irreversibly change the variable class are apparent (e.g. in the context of Canadian Football League, the sub-topic of interest that a user is tweeting may suddenly switch from one game to another game each week).

In contrast to sudden drift, gradual concept drift (depicted in Figure 3.1(b)) occurs when there is a smooth transition in the distribution class of the variable (e.g. in the context of Canadian Football League, relevant sub-topic of a user change from one game to another game, while the user does not switch abruptly, but rather keeps going back to previous interest for some time).

As the concepts changes over time, there may be instances where a concept will reoccur (depicted in Figure 3.1(c)), this is called reoccurring drift. A concept can reoccur either suddenly, or gradually. Reoccurring drift is not certainly periodic as seasonality concept. It is not clear when the source might reappear and that is the main difference from seasonality concept used in the statistic [12].

(a) Sudden

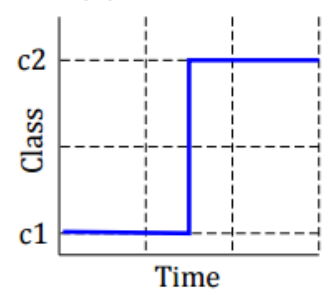

(b) Gradual

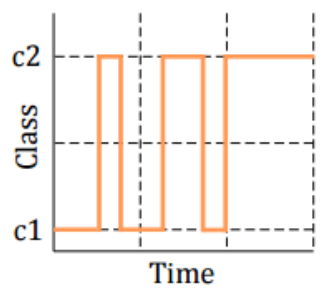

\section{(c) Recurring}

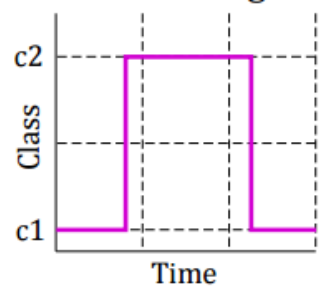

Figure 3.1: Types of concept drift in time series data streams. 
The sudden and gradual drifts that are rare in the underlying data pattern represents anomalous behaviour. Such anomalous behaviour is of interest to the analyst benefiting from the work in this thesis. However, the reoccurring drift that is either sudden or gradual is considered normal after these concepts repeatedly appear and are not rare anymore in the underlying data pattern. In order to detect such rare anomalous behaviour, two types of anomalies are formally defined in the context of this Thesis.

Definition 3.1.1 (Candidate Anomaly). A data point $\left(d_{t}\right)$ is a candidate anomaly $\left(c_{t}\right)$ if its value deviates from the values of other data points in the local context by a factor of at least $\tau_{c}$. The threshold value $\tau_{c}$ is a user defined parameter.

Definition 3.1.2 (Legitimate Anomaly). A candidate anomaly is legitimate $\left(l_{t}\right)$ if its value deviates from the values of other previously detected candidate anomalies within some limited timeframe (called a window), by a factor of at least $\tau_{l}$. The threshold value $\tau_{l}$ is a user defined parameter.

In the above definitions $\tau_{c}$ and $\tau_{l}$ are two tunable factors. $\tau_{c}$ is the threshold to identify data points as candidate anomalies, whereas $\tau_{l}$ is the threshold to identify candidates as legitimate anomalies.

Figure 3.2 illustrates an example of the candidate and legitimate anomalies detected in a synthetic time series data stream of tweets. These time series evolves from Class 1 to Class 5 of the underlying data distribution. The significance of these classes is to measure the change in data distribution. Between time T1 and T2, statistically significant changes in the data begin to appear gradually, resulting in gradual concept drift from Class 1 to Class 2. As these are the first occurrence of Class 2 gradual concepts between $\mathrm{T} 1$ and $\mathrm{T} 2$, these changes are rare and detected as both candidate and legitimate anomalies. However, after time T2, Class 2 concepts 


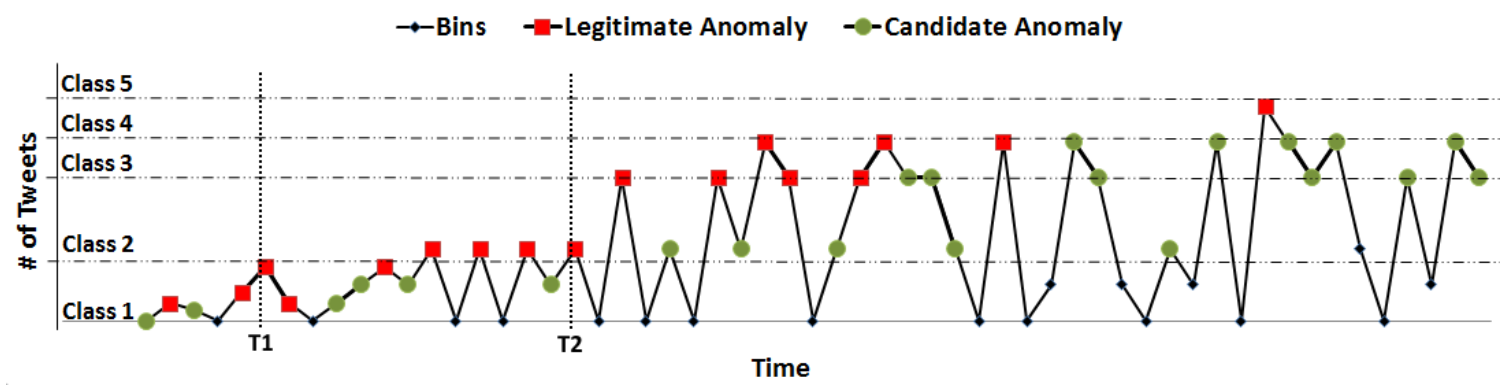

Figure 3.2: Synthetic time series data streams, presenting the legitimate and candidate anomalies.

are detected only as candidate anomalies and not as legitimate anomalies. This is because the reoccurring nature of the Class 2 concept became apparent in the data. Similarly, the gradual shift in the data distribution between Classes 3, 4, and 5 are detected as legitimate anomalies until they appear to be rare and the repetition becomes apparent in the time series, after which the candidate anomalies are no longer providing new information about the pattern.

\subsection{Overview of the RSAD Approach}

Figure 3.3 gives an overview of the sentiment-based anomaly detection process presented in this Thesis. Assume that a Twitter data stream has been configured to provide tweets based on some user-specified query (e.g., "\#tdf"). The RSAD approach processes the input data stream in two main steps: preprocessing and twostage real-time anomaly detection (TRAD).

\subsubsection{Step 1: Preprocessing}

As each new tweet arrives from the data stream, two preprocessing steps are performed. The preprocessing steps transform the input Twitter data stream into three 


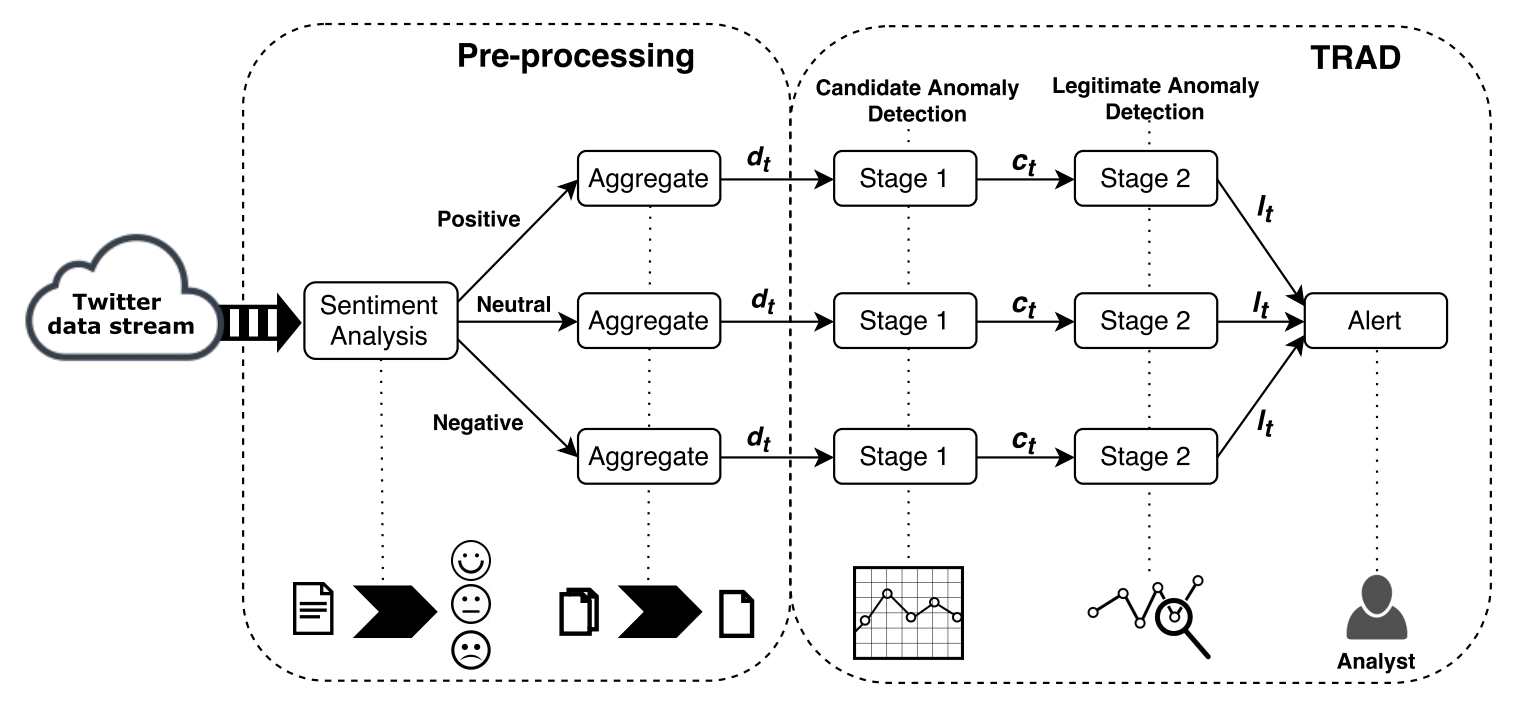

Figure 3.3: Real-time multi-stage analysis on Twitter data stream: pre-processing and anomaly detection stages, in proposed RSAD approach

separate aggregated time series data streams, which substantially reduces the problem of detecting sentiment-based anomalies to the more familiar problem of detecting anomalies in a time series. In the first preprocessing step, sentiment analysis is performed using Sentiment 140 to classify tweets as positive, neutral, or negative [42] as they arrive. Sentiment 140 was designed specifically to address the short and cryptic nature of English language tweets.

In the second preprocessing step, temporal binning is performed over the classified tweets. The granularity of this temporal binning is based on temporal bin length and affects the sensitivity of the RSAD method to small-scale vs. large-scale anomalies. The temporal bin length can be set based on an expectation of the velocity patterns of the tweets for the given query. Since the goal is to analyze these data streams based on tweet frequency, once the classification and temporal binning are performed, the actual contents of the tweets can be discarded. All that remains is the number of positive, neutral, and negative tweets that were seen in each temporal bin. These frequency counts serve as the data points for the TRAD stage. 


\subsubsection{Step 2: Two-stage Real-time Anomaly Detection}

The two-stage real-time anomaly detection (TRAD) step simultaneously and independently analyzes the number of tweets in the temporal bins for each of the positive, neutral, and negative sentiment streams in order to determine if any of these should be considered anomalies. To maintain approximately real-time performance, the analysis of one temporal bin must happen sufficiently quickly so that it is complete before the next bin of data is generated. For example, if the temporal bin length is set at two minutes, this process must be completed in less than two minutes.

In contrast to analyzing a stream of the number of tweets, the independent analysis of each sentiment stream is important. The independent analysis allows to detect the change in the number of tweets in one class (e.g., increases in the positive tweets) even if it is masked by an inverse change in another class (e.g., decreases in the negative tweets). In such cases, there will be no apparent change in the total number of tweets, whereas changes in the sentiment classes could be inverse.

A two-stage process is employed for real-time anomaly detection: first, the candidate anomalies are identified based on their difference from the local context, and second, these candidates are examined to see if they are legitimate anomalies by comparing them to other candidate anomalies that have been seen recently. The following two subsections explain several approaches that can be used for these two respective stages.

\section{Stage 1: Candidate Anomaly Detection}

To detect candidate anomalies among a time series of data points, we consider a deviation-based approach using two possible methods for determining the average of the previously seen data points: exponentially weighted moving average (EWMA) [36] and probabilistic exponentially weighted moving average (PEWMA) [13]. While each 
of these approaches has been used to detect outliers in streaming data in separate contexts $[13,34]$, it is not clear which is most appropriate for detecting sentimentbased anomalies in Twitter data.

In the deviation-based approach, an anomaly score of a data point is calculated that represents its deviation from the mean of the data points in the neighborhood. In the streaming context, the local neighbors of an newly arrived data point are the ones that recently arrived (past data) because the following data points (future data) have not yet been received. The candidate anomaly score (CAS) for a data point $d_{t}$ at time $t$ is evaluated using the following formula:

$$
C A S\left(d_{t}\right)=\frac{\left|d_{t}-\mu_{c(t-1)}\right|}{\mu_{c(t-1)}}
$$

where $\mu_{c(t-1)}$ is the mean of the past data points at time $t-1$. CAS was adapted from the A-ODDS technique [40]. If the CAS of the current data point is near zero, the point is close to the other data points in the neighborhood. If the CAS of the current data point is large, then the data point is significantly larger or smaller than the other data points. In order to label the current data point as a candidate anomaly, the CAS should be larger than the standard deviation of the recently seen data points by some factor. The threshold condition for a data point $d_{t}$ to be so labeled is given as:

$$
C A S\left(d_{t}\right)>\tau_{c} * \sigma_{c(t-1)}
$$

where $\sigma_{c(t-1)}$ is the standard deviation of the past data points at time $t-1$ and $\tau_{c}$ is a parameter that can be set by a domain expert according to the type of the data stream. A lower value of $\tau_{c}$ increases sensitively to anomalies with low level of granularity, whereas a higher value increases sensitivity to high level of granularity.

A data profile represents the statistics and information about the data with respect 
to the local context (recent history of data points) or the global context (complete history of data points). Here $\mu_{c(t)}$ and $\sigma_{c(t)}$ are the data profile representing the local context, and these need to be updated for each new data point $d_{t}$. A naïve approach is to consider all the data points over the last $t$ periods. Then using these data points, the data profile in the local context can be updated with the incremental mean and standard deviation method as given in Equations 2.5 and 2.6. However, when using this method to update the data profile, it will consider all the data points and it would be difficult to calculate the data profile for the local context incrementally without maintaining the list of all data points (i.e., to calculate the mean and standard deviation for the recent history of data points).

Another approach is to use the exponentially weighted moving average (EWMA) [36] and incrementally update $\mu_{c(t)}$ and $\sigma_{c(t)}$ as given in the following equations:

$$
\begin{gathered}
\mu_{c(t)}=\alpha_{E W M A} \cdot \mu_{c(t-1)}+\left(1-\alpha_{E W M A}\right) \cdot d_{t} \\
\sigma_{c(t)}=\alpha_{E W M A} \cdot \sigma_{c(t-1)}+\left(1-\alpha_{E W M A}\right) \cdot\left|d_{t}-\mu_{c(t-1)}\right|
\end{gathered}
$$

Here $0<\alpha_{E W M A}<1$ is the decay weighting factor. The $\alpha_{E W M A}$ parameter controls the weight distribution between the new data point $d_{t}$ and the old mean $\mu_{c(t-1)}$. A value of 0 implies no weight on the history, while a value of 1 implies all weight on the history. Algorithm 3.1 shows the procedure to update data profile for local context using EWMA.

An inherent assumption with the EWMA approach is that the mean is changing gradually with respect to the exponential weighting parameter $\alpha_{E W M A}$. Thus, a significant change in $d_{t}$ will result in a significant increase in $\mu_{c(t)}$ and an even greater increase in $\sigma_{c(t)}$. To increase resiliency against such changes in $d_{t}$, the value of the weighting parameter $\alpha_{E W M A}$ can be dynamically adjusted. More precisely, if 


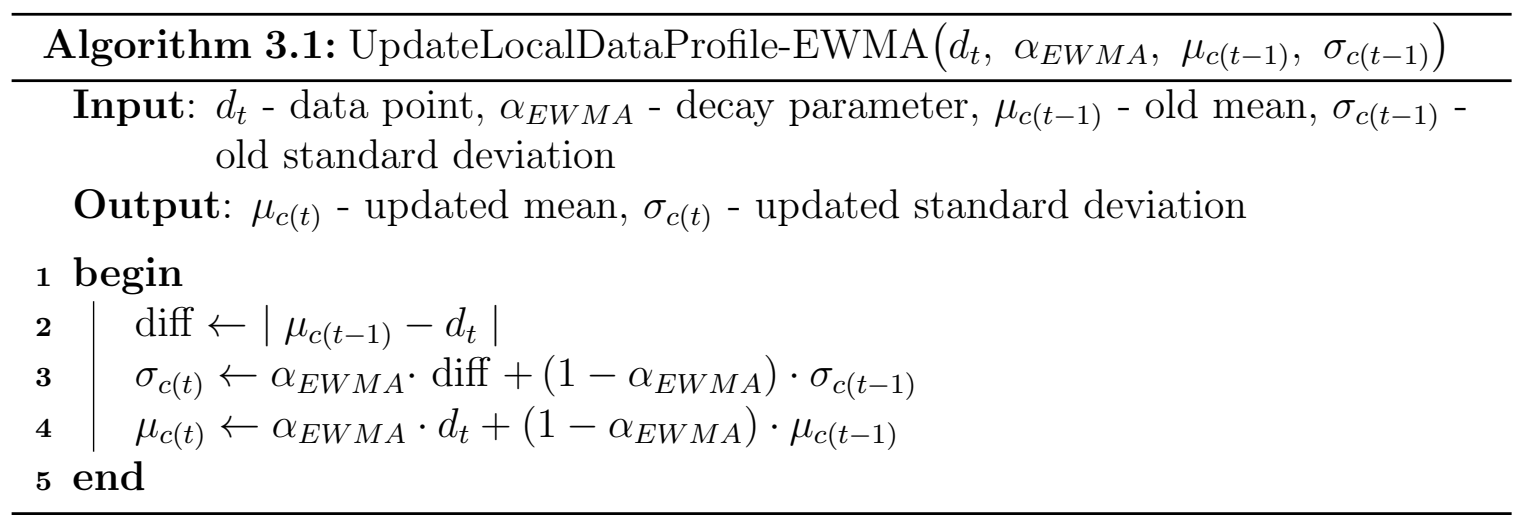

$d_{t}$ changes with respect to recent data points, then a higher weight $\left(\alpha_{E W M A}\right.$ close to 1) should be given to recent data points; otherwise, more weight should be given to $d_{t}$.

Another approach that probabilistically adjusts the weighting parameter is probabilistic exponential moving average (PEWMA) [13]. This approach adjusts the weighting parameter based on the probability of occurrence of the value of the current data point. The probabilistic weighting parameter is given as $\alpha_{P E W M A}=$ $\alpha_{E W M A}\left(1-\beta P_{t}\right)$, where $P_{t}$ is the probability of occurrence of $d_{t}$ and $\beta$ is the weight placed on $P_{t}$. The parameter $\alpha$ is multiplied by $\left(1-\beta P_{t}\right)$ to reduce the influence of an abrupt change in $d_{t}$ on the moving average.

The probability density estimator equation with the standard normal distribution for $P_{t}$ is given as:

$$
P_{t}=\frac{1}{\sqrt{2 \pi}} \exp \left(-\frac{Z_{t}^{2}}{2}\right)
$$

While evaluating $P_{t}$ for the current data point $d_{t}$, it may happen that $P_{t} \rightarrow 0$, if $\sigma_{c(t-1)} \rightarrow \infty$. To avoid such situations, normalization is applied to the input data points to obtain a zero-mean and unit standard deviation random variable $Z_{t}=$ $\left(d_{t}-\mu_{l}\right) / \sigma_{l}$. The factor $\frac{1}{\sqrt{2 \pi}}$ is the constant height and it is selected to normalize $P_{t}$ such that $0<P_{t}<\frac{1}{\sqrt{2 \pi}}$. The drawback of considering the standard normal 
distribution is that for larger values of $d_{t}, P_{t} \rightarrow 0$. However, our approach does not require that the deviation of $d_{t}$ be large, as long as it is sufficiently deviated from the underlying data distribution. By adjusting equation 3.22, with the probabilistic weighting factor [13], we get:

$$
\mu_{c(t)}=\alpha_{P E W M A} * \mu_{c(t-1)}+\left(1-\alpha_{P E W M A}\right) * d_{t}
$$

Algorithm 3.2 shows the process of updating a data profile for a local context using PEWMA. The value for the decay weighting factor is updated (line 6) based on the probability of occurrence of the input data point $d_{t}$. Then, Algorithm 3.1, which computes EWMA, is called with the updated weighting factor (line 7). Algorithm 3.1 depends on the decay factor passed as a parameter, whereas Algorithm 3.2 generates the decay factor from the data distribution of recent data points.

Figure 3.4 shows a time series data stream of positive sentiment tweets. The figure illustrates the impact of a sudden change in $d_{t}$ (high peaks) on the estimated standard deviation (above and below the mean) when evaluated with EWMA versus PEWMA. EWMA remains high for an extended period, resulting in false negatives

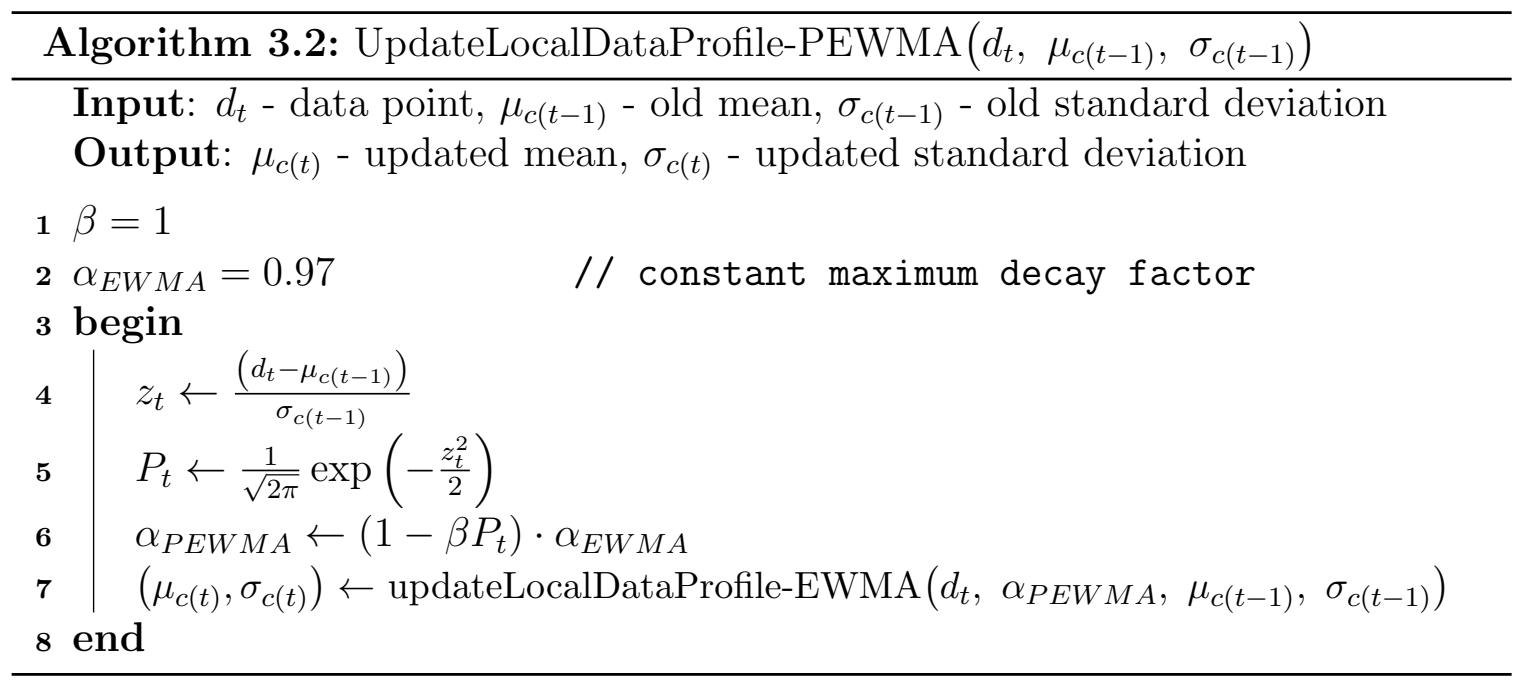




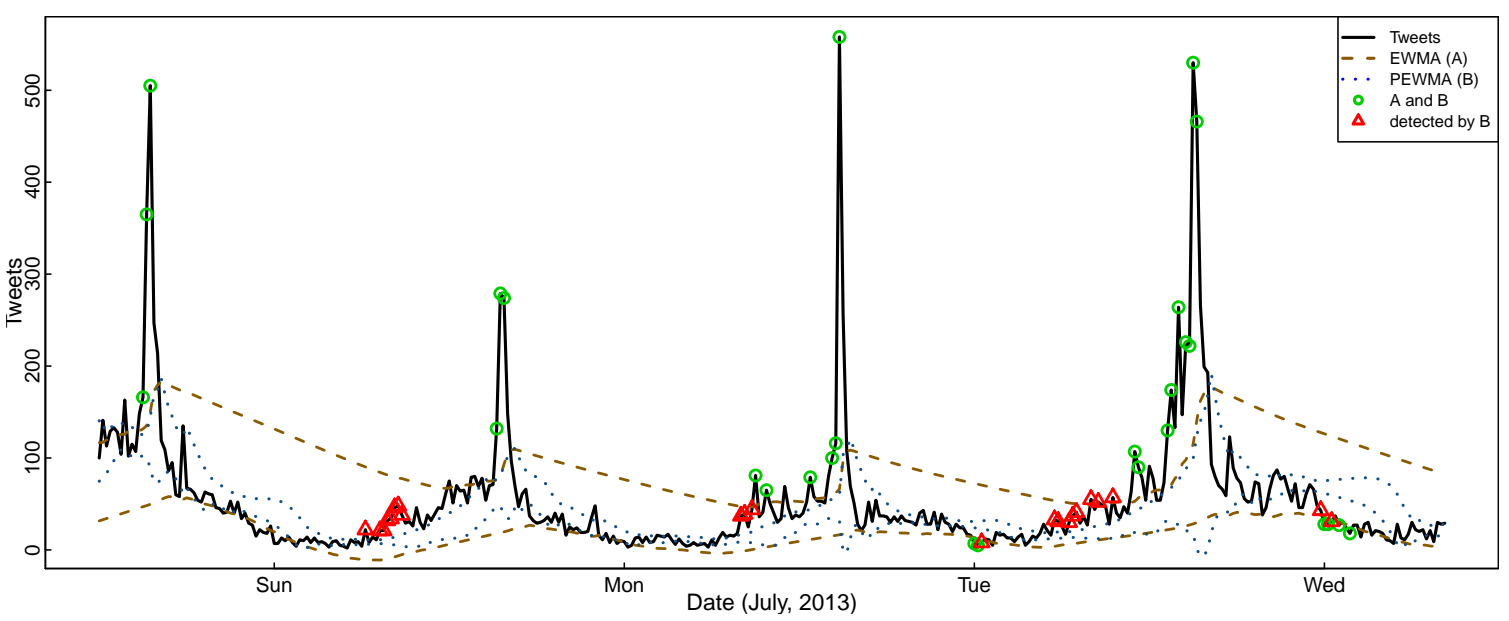

Figure 3.4: Candidate anomalies detected using one standard deviation estimated (above and below mean) with EWMA and PEWMA respectively (temporal aggregation of 15 minutes).

(triangles) when analyzing for candidate anomalies. In contrast, the standard deviation as estimated by PEWMA, quickly adjusts after the sudden change in $d_{t}$ as the large peak is identified.

\section{Stage 2: Legitimate Anomaly Detection}

To detect whether a candidate anomaly should be considered a legitimate anomaly, we use a one-sided sliding window of length $W_{t}$ (e.g., 6 days). In contrast to maintaining all past data points in a sliding window that fall in the window length, which is the conventional way, only those data points are maintained that are identified as anomalies in Step 1. As the sliding window moves forward with the arrival of a new data point, the expired anomalous data points from the tail of the sliding window are removed.

A window-based deviation approach is considered using two possible methods for determining the deviation of the data points in the window: standard deviation (STD) based on the simple arithmetic mean, and median absolute deviation (MAD) based on the median. While each approach has been used to detect outliers in static time 
series data $[33,36]$, it is not clear which is most appropriate for a sliding window.

To determine whether a candidate anomaly should be considered legitimate anomaly, the legitimate anomaly score (LAS) is calculated. The LAS of a data point represents its deviation from the mean of the candidate anomalies in the window. For the current data point $d_{t}$, the equation for LAS is computed as:

$$
L A S\left(d_{t}\right)=\frac{\left|d_{t}-\mu_{l(t-1)}\right|}{\mu_{l(t-1)}}
$$

where $\mu_{l(t-1)}=\sum_{i=\left(t-W_{t}\right)}^{t} d_{i}$ (Equation 2.3) and $W_{t}$ is the window length. In order to account for the data points that are in the window at time $t$, only the past $W_{t}$ points are considered. LAS gives the relative distance of $d_{t}$ with respect to the mean of the candidate anomalies in the window.

The significance of the LAS value is similar to that of CAS in Equation 3.20. The value of the LAS for data point $d_{t}$ should be sufficiently large in order to label it as a legitimate anomaly. The cutoff condition is given as:

$$
L A S\left(d_{t}\right)>\tau_{l} * \sigma_{l(t-1)}
$$

where $\sigma_{l(t-1)}=\sqrt{\frac{1}{W_{t}} \sum_{i=\left(t-W_{t}\right)}^{t}\left(d_{i}-\mu_{l(t-1)}\right)^{2}}$ (Equation 2.4), which is the standard deviation (STD) estimated from the simple arithmetic mean of the recent candidate anomalies in the window. $\tau_{l}$ is a threshold factor for legitimate anomalies, which is similar to $\tau_{c}$ in Equation 3.21.

At each step of the algorithm the mean $\mu_{l(t)}$ and standard deviation $\sigma_{l(t)}$ are updated with respect to the sliding window. Algorithm 3.3 shows the procedure to update the data profile representing the sliding window using the STD.

The number of data points in the sliding window will be sufficiently small, since we are only maintaining the candidate anomalies, as opposed to all the data points. 


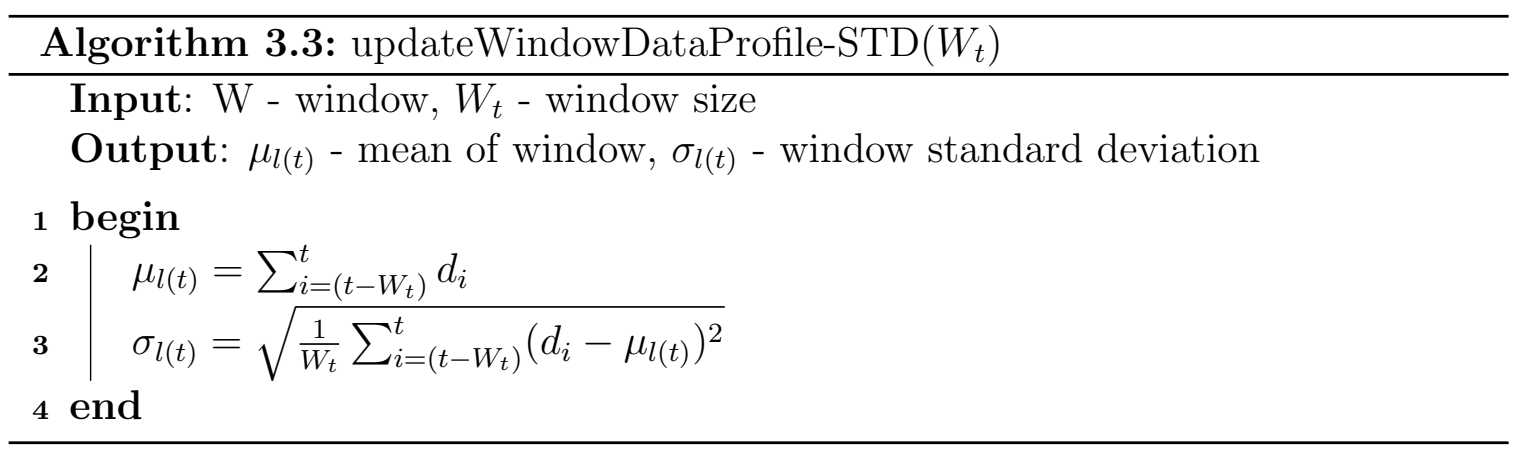

In the case where the sample data set is relatively small, the standard deviation technique is strongly affected by the presence of extreme anomalies [33]. In such a scenario, robust statistical techniques, such as median and median absolute deviation (MAD), which are resilient with respect to extreme values, are recommended [33]. The median of the sliding window of previously detected candidate anomalies is given as: $M_{t}=M_{i=\left(t-W_{t}\right)}^{t}\left(d_{i}\right)$. Moreover, the median absolute deviation (MAD) is calculated as:

$$
M A D=\beta \cdot M_{i=\left(t-W_{t}\right)}^{t}\left(\left|d_{i}-M_{t}\right|\right)
$$

The process for updating the data profile representing the sliding window using MAD is shown in Algorithm 3.4. First, the median of the window is evaluated (line 4) and then the MAD of the window is computed using Equation 3.28 (line 5).

Figure 3.5 shows an example of a time series data stream of negative sentiment

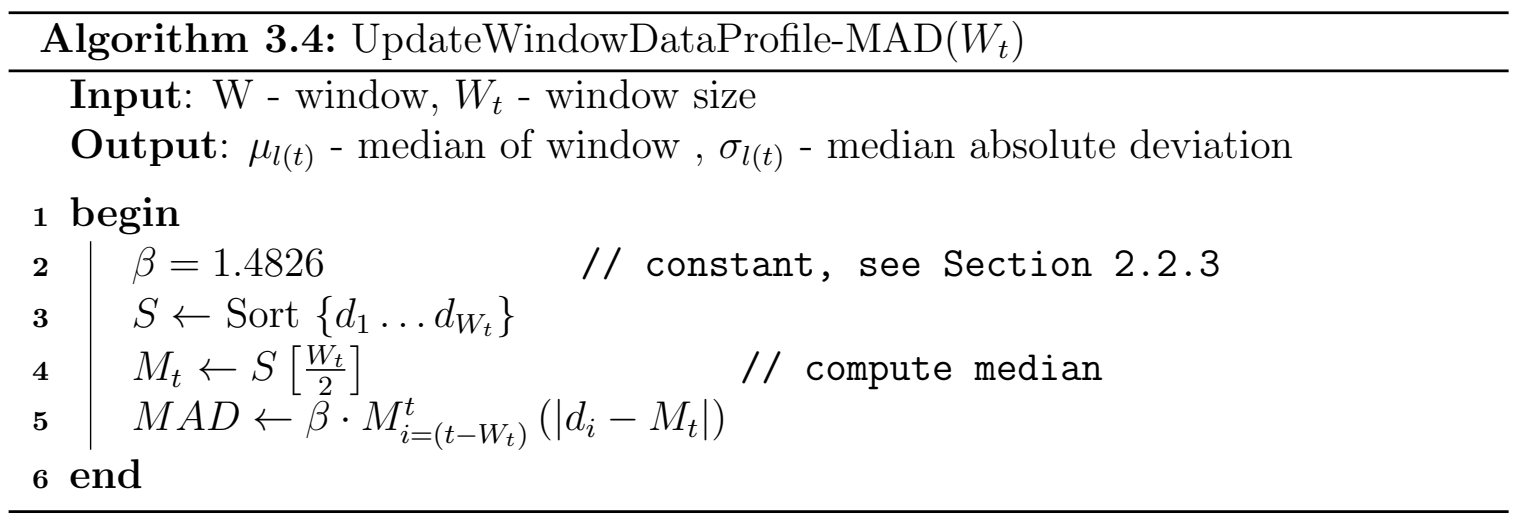




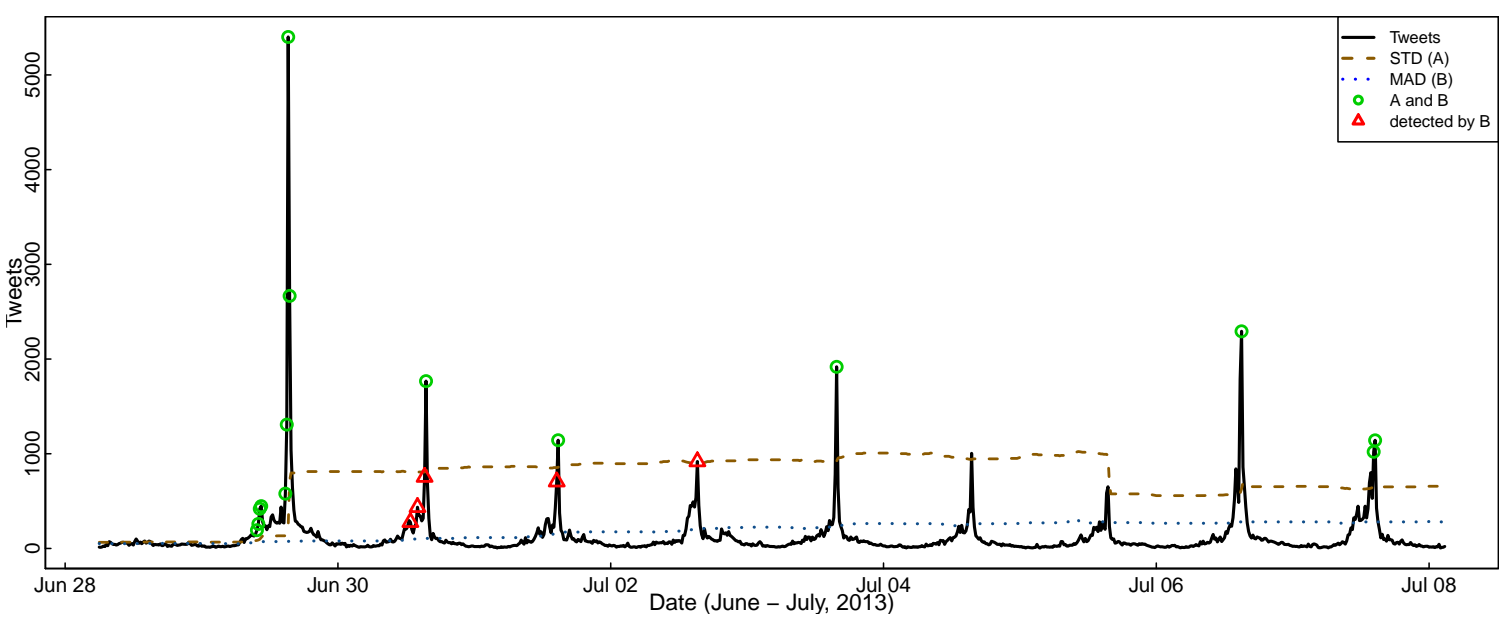

Figure 3.5: Candidate anomalies identified as legitimate anomalies using STD and MAD, estimated with the simple arithmetic mean and median respectively (temporal aggregation of 15 minutes)

tweets and $W_{t}=6$ days. The figure illustrates the effect of large peaks on the estimated mean and median values. The mean of the window increases abruptly when a large peak enters the window on June 30. The mean drops when this peak is removed from the sliding window after six days (July 6). This shows that the presence of extreme values changes the mean dramatically. During this period, the standard deviation estimation (above and below the mean) failed to identify the true legitimate anomalies (triangles). Whereas, the median absolute deviation estimation (above and below the median) was not affected by the extreme peaks, and the legitimate anomalies were found effectively.

\subsection{The Online TRAD Algorithm}

Algorithm 3.5 presents the streaming and steady-state version of the proposed TRAD approach. In order to operate in steady-state, it is necessary to first train the model on initial observations during start-up. The model will be trained for initial period $T$, i.e., until $t \leqslant T$ (line 8 ). It is important to note that if anomalies are 


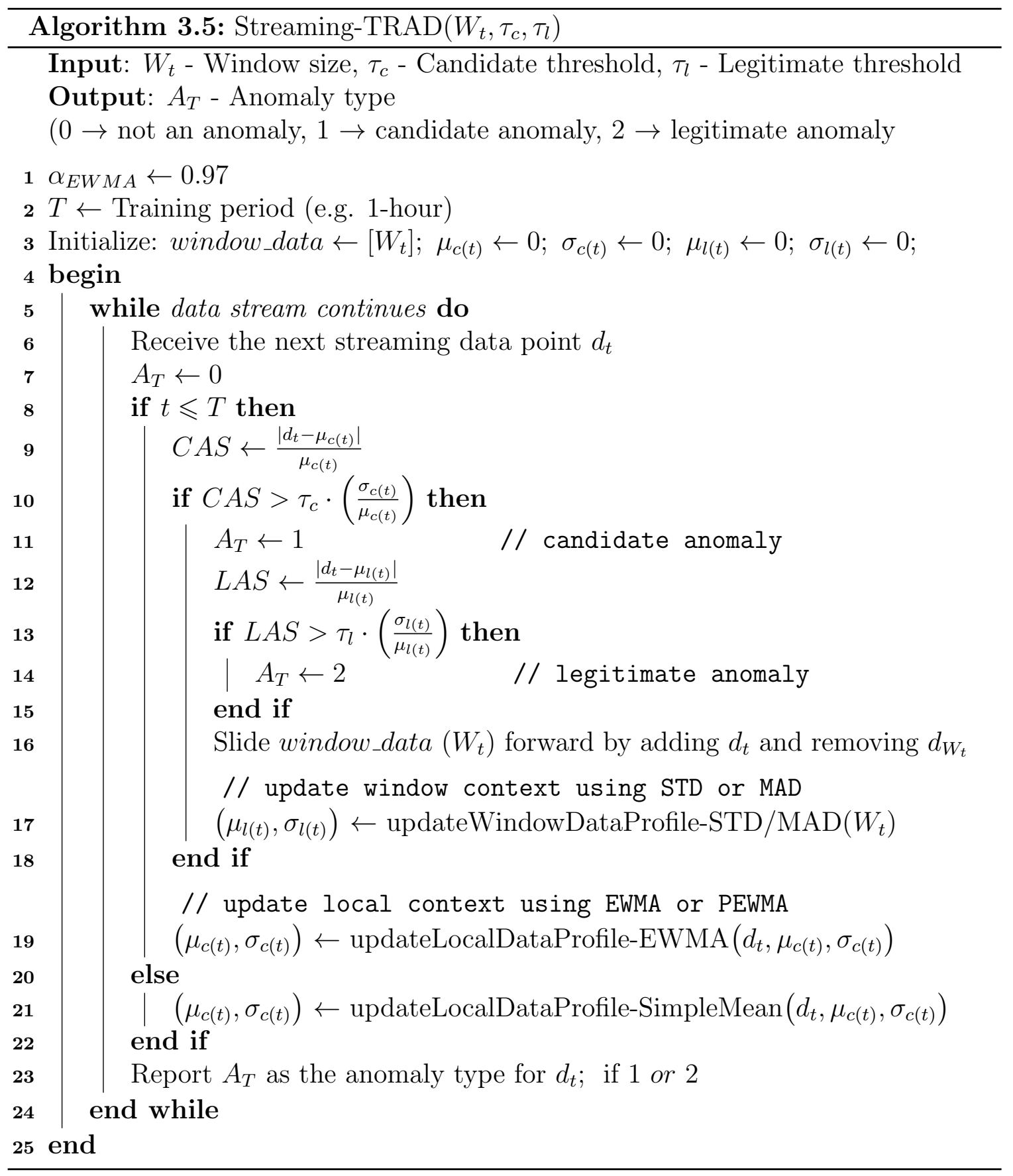

present during the training period, it may take longer to neutralize their effect on the model being learned, as EWMA based methods have the tendency to gradually forget history. This can be overcome by computing the local data profile model using the exact mean and standard deviation (line 21) of the data during the training period 
$(t<T)$. The value for the training period depends on the underlying data distribution (e.g., for a bursty time series, a shorter training period can capture the normal data, however for a smooth time series the training period must be longer to capture the normal data). If the nature of the underlying data is unknown, the optimal training period value can be chosen as close to the window size $W_{t}$ as possible, such that the window can be initialized.

The algorithm accepts three user defined parameters: window size $\left(W_{t}\right)$, candidate threshold $\left(\tau_{c}\right)$, and legitimate threshold $\left(\tau_{c}\right)$, which are associated with each query. The output of the algorithm is simply a flag that represents the type of anomaly detected (i.e., candidate or legitimate anomaly or no anomaly at all). As each data point $d_{t}$ arrives (line 6), first the anomaly score CAS is computed (line 9). The CAS is then compared to the standard deviation. A data point is considered a candidate anomaly if its CAS goes beyond the standard deviation by the factor of the candidate threshold (line 10 and 11). If the condition in line 10 is true, i.e., $d_{t}$ is a candidate anomaly, then we compute legitimate anomaly score LAS to check if it is a legitimate anomaly. Similarly, the LAS is compared to the standard deviation of the window (line 13). A data point is considered a legitimate anomaly (line 14) if its LAS goes beyond the standard deviation by the factor of the legitimate threshold. If the data point $d_{t}$ is identified as any one of the above anomalies, it is pushed into the sliding window of recent anomalies (line 16). Finally, the mean and standard deviation representing the data profile in the two different contexts, local (line 19) and window (line 17), are updated to account for the new data point. An alert is generated (line 23) only if a legitimate anomaly is detected (i.e., $A_{t}=2$ ) and optionally, for a candidate anomaly if requested by the analyst.

The proposed algorithm does not require the entire data set; instead it works online and incrementally. As this is an online algorithm, there is no return statement 
in the logic, instead the algorithm will keep processing the incoming data points from the data stream until it is terminated manually. We will discuss the performance of the above algorithm in the results section.

Note that for the purpose of conducting experiments, lines 19 and 17 are changed to use appropriate functions (EWMA or PEWMA for local context and STD or MAD for window context). This is because for the evaluation of these alternative approaches, the experiments are conducted for each combination of these methods to detect the candidate and legitimate anomalies.

\subsection{Computational Complexity}

As this thesis proposes an algorithm for the real-time analysis of data streams, it is crucial to consider its performance in terms of execution time. We consider the RSAD algorithm to be sufficiently efficient if it is able to analyze the current collection of binned tweets before the next one is generated, making it suitable for real-time performance depending on the user selected binning interval.

In terms of computational complexity, the calculations used to determine the candidate anomalies are linear due to the incremental nature of calculating EWMA and PEWMA. When determining whether or not a candidate anomaly is a legitimate anomaly, it is necessary to loop over all of the candidate anomalies in the current window for calculating STD and MAD. As such, this second step has a complexity of $O(n)$ (Equation 2.6), where $n$ is the maximum number of potential candidate anomalies. For example, given a window size of 6 days and an aggregation interval of 15 minutes, the worst-case value for $n$ is 576 . Clearly, with these settings, the

approach can be considered to run in real-time. Even with a high velocity data stream, as long as the aggregation interval is at least one minute, the approach will 
be able to keep up with the inflow of data on a sufficiently fast computer system.

\subsection{Implementation in the Apache Storm Framework}

An important contribution of this Thesis is to implement data collection and anomaly detection techniques together in a distributed real-time streaming framework. Streaming algorithms that analyze data streams are difficult to implement on their own without making use of a streaming framework. This is because there are critical challenges when processing data streams efficiently such as fault-tolerance, scalability with distributed processing, low latency computation, and efficient storage. All of these must be addressed to ensure that the overall system does not fail in the cases where data streams are generated at a faster rate than the system can handle.

Recently, the increasing need for real-time computation on data streams has led the open-source community to develop stream processing frameworks, which are designed specifically to overcome the above mentioned challenges. In this work, we utilize a stream processing framework called Apache Storm [47]. Storm is a distributed, highly scalable, fault-tolerant framework for real-time analysis of streaming data. Storm works by specifying a streaming topology in terms of spouts (data sources), tuples (data objects), and bolts (tuple processing units). While technologies like Hadoop [32] and Spark [55] process batches of static data, Storm is designed to continuously analyze an incoming stream of data. Although Spark has a streaming API, Storm is purpose-built for streaming analysis. A detailed survey of available open-source stream processing frameworks is given by Bifet et al. [20]. 


\subsubsection{Concepts in Storm}

In order to understand the implementation details that are provided in Section 3.5.2, we list the core concepts in Storm [4] as illustrated in Figure 3.6.

Topology: The overall logic and data flow for a real-time application is packaged into a Storm topology. A Storm topology is a graph of spouts and bolts that are connected with stream groupings. A topology is analogous to a MapReduce job [17], which processes a stream of data in batches. However, one key difference is that a MapReduce job eventually finishes, whereas a topology runs forever (or until stopped manually).

Tuple and Stream: A Tuple is the core data structure in Storm. A tuple is a list of key-value pairs, where the values are dynamically typed, i.e., the types of the fields do not need to be declared and they can be of any type. It is also a serializable object, as it needs to be serialized and de-serialized when distributed between tasks. A stream is an unbounded sequence of tuples that is processed and created in parallel and distributed fashion. Spouts and bolts interact with each other through streams.

Spouts: A spout is the source for a stream in the topology. Spouts read data from an external source, convert the data into a tuple, and then emit the tuple into the topology (e.g. log file, Twitter API). They are the starting point in the topology, from where the stream is initiated. Spouts emit a stream to subsequent bolts for

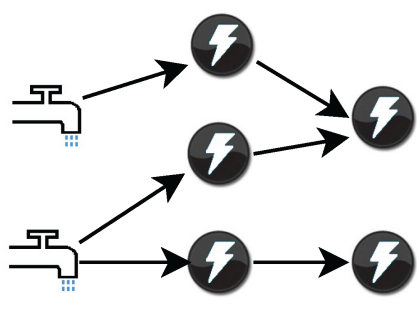

Topology

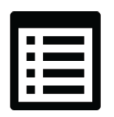

Tuple

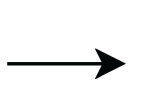

Stream

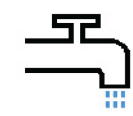

Spout

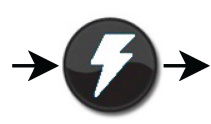

Bolt

Figure 3.6: Core concepts in Apache storm, adapted from [4] 
further processing.

Bolt: All the processing in the topology is done in bolts. Bolts provide a variety of services such as filtering aggregations, performing joins, communicating with the database, and more. The input to a bolt is a stream which is emitted from a spout or another bolt. Bolts are capable of performing simple stream processing. Complex stream processing often requires multiple steps and thus multiple bolts. For example, processing a stream of tweets into a stream of trending topics requires at least three steps: a bolt to split text into words, and one or more bolts to keep a rolling count of each word, and a bolt to stream out the top- $n$ topics. After processing a stream, a bolt can emit the processed tuples to subsequent bolts or store them in a database.

Parallelism and Stream Grouping: Parallelism of topology components is a crucial factor that needs to be configured to ensure that the overall stream processing performance is adaptive to variations in the data stream velocity. Each spout or bolt can be configured to execute as many instances as needed across the topology. Each instance corresponds to one thread of execution and the stream grouping defines how the input stream should be partitioned among the multiple instances of a bolt. In other words, if the bolt is not paralleled (unique), the input stream will be processed by a single thread. With parallelism, the input stream will be partitioned among multiple threads of instances to execute the same task for the bolt simultaneously. Stream grouping defines the flow for partitioning the stream. Example of stream grouping includes shuffle grouping, fields grouping, and direct grouping. Storm has dynamic parallelism, where a minimum and maximum preference for the degree of parallelism can be specified. If the workload is less such that one instance can handle, minimum parallelism is done for the bolt's tasks. If the workload increases, the number of threads are dynamically increased to less than or equal the maximum preference. 


\subsubsection{Twitter Analytics Topology}

The design of TAT for the overall system is shown in Figure 3.7. When receiving the Twitter data stream, the Twitter Spout processes each tweet object and extracts the required fields including: tweet timestamp, text, user, geolocation, and retweet count. A tuple object is created from the extracted fields and emitted by a spout for further processing. First, this tuple object is preprocessed for sentiment classification by the preprocess bolt. Then the multistage bolts process these sentiment classified tuples and cooperate with each other to implement the anomaly detection algorithm and the data collection process. The anomaly detection approach (TRAD) presented in Section 3.3, was described in the context of analyzing a single query, however Twitter Analytics Topology is designed such that anomaly detection can be scaled to analyze multiple queries simultaneously.

The overall workload is distributed between three modules: Preprocess, Monitor, and Storage, which processes the stream of tuples synchronously. The Preprocess module classifies the tuples into specific query and the sentiment by analyzing the

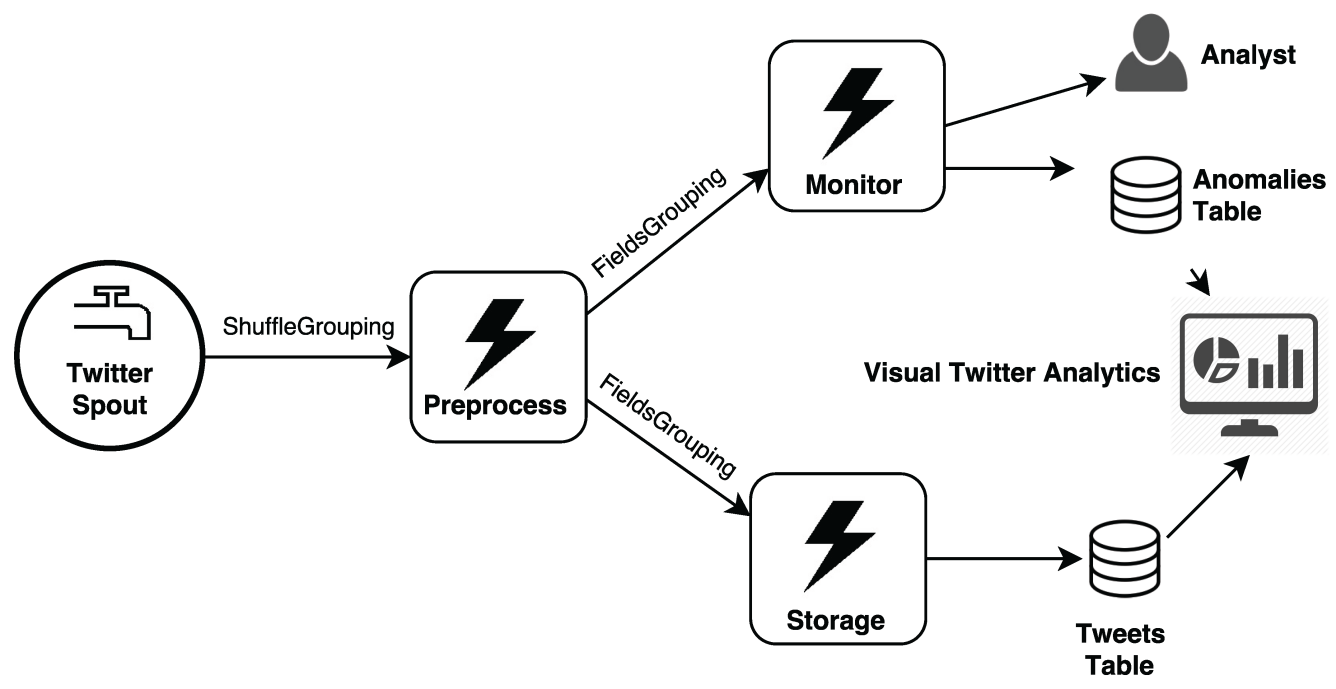

Figure 3.7: The design logic and data stream flow for overall system packaged in Twitter Analytics Topology (TAT) 
tweet text. Here, as a preprocessing stage, two new fields are appended to each tuple, query and sentiment, which are then forwarded to the Monitor and Storage modules as a stream. The Monitor module analyzes the tuples to detect anomaly by implementing the proposed TRAD approach. For each identified anomaly, an email is sent to the analyst and the anomaly details are stored in the Anomalies table. The Storage module collects the tweet data from the tuples and then stores them in the Tweets table. This process is done in batches to avoid frequent access to the database. The stored data in the Anomalies and Tweets tables are then used for any application that performs analyses of Twitter data, such as a visual twitter analytics application $[26,27]$.

\subsubsection{Integration of Continuous Input Twitter Streams}

The Twitter data stream that provides every message from every user in real-time is called the Streaming API [50]. To capture and analyze the massive amount of real-time tweet data delivered by these Twitter Streaming API, we have redesigned

Storm spout's operating logic as illustrated in Figure 3.8 as a Twitter spout. An open source library called Twitter4J [49] is used to make the connection with the Streaming API. While connecting, a list of queries from the queries table is provided.

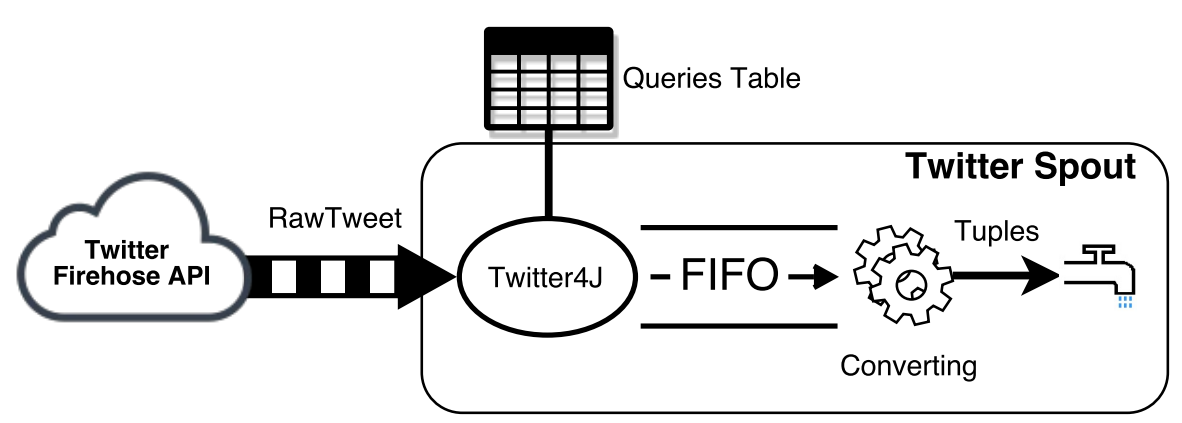

Figure 3.8: Internal working of the redesigned Storm spout for processing a Twitter data stream 
These queries in the database are the ones specified by the analyst. The queries table structure is shown in Table 3.1. Once the connection is authenticated, Twitter will keep pushing the raw tweet objects (as JSON data) in real-time to the spout. The spout then queues the raw tweets to an in-memory first in first out (FIFO) queue. The raw tweets are eventually popped out from the queue and processed to generate tuple objects with the selected fields. The queue is used as a buffer, in the case where the raw tweets from the Firehose API are arriving faster than the spout can process them. Finally, the generated tuple objects will be sent to subsequent bolt components as an internal stream.

\subsubsection{Preprocessing the Stream}

The Preprocess module in the topology performs two classification tasks, query and sentiment classification as shown in Figure 3.9. First, query classification is performed by the Query Analysis bolt. A tuple that is emitted from the Twitter Spout contains tweet information. However specific information about the query that is associated with this tweet is not known, as Twitter does not specify this information explicitly in the response. In order to categorize a stream of tweets into those that are produced from multiple queries, each tweet is classified by matching the tweet content with the list of queries from the Queries table (Table 3.1) using regular expressions. A tweet can be classified into one or more queries and tagged

Table 3.1: Queries table used by the Storm topology

\begin{tabular}{|l|l|}
\hline \multicolumn{1}{|c|}{ Fields } & \multicolumn{1}{c|}{ Type } \\
\hline Query & String (name of the query e.g. \#earthquake) \\
\hline Window_Size & Integer (sliding window length in minutes) \\
\hline Legitimate_Threshold & Decimal (between 1 and 5) \\
\hline Candidate_Threshold & Decimal (between 1 and 5) \\
\hline Aggregation_Factor & Integer (duration in minutes) \\
\hline
\end{tabular}


accordingly.

Second, sentiment classification is performed by the Sentiment Analysis bolt. The system utilizes a third party API called Sentiment 140 [42] which classifies the tweet text into negative, neutral, and positive classes. In order to avoid making redundant API requests for each tuple, a list of tweet text is prepared by extracting the text from a small batch of tweets. A request to the API is made with the list of tweet text for bulk processing and it responds with the list of codes corresponding to the sentiment class.

The Query Analysis bolt is configured for parallel execution. However, the Sentiment Analysis bolt is not parallelized because the number of requests to the Sentiment140 API is restricted. Having a single instance of Sentiment Analysis bolt makes it easier to keep track and enforce the number of requests being made to the Sentiment 140 API. Finally, the tuple classified by query and sentiment is emitted further to the Monitor and Storage modules.

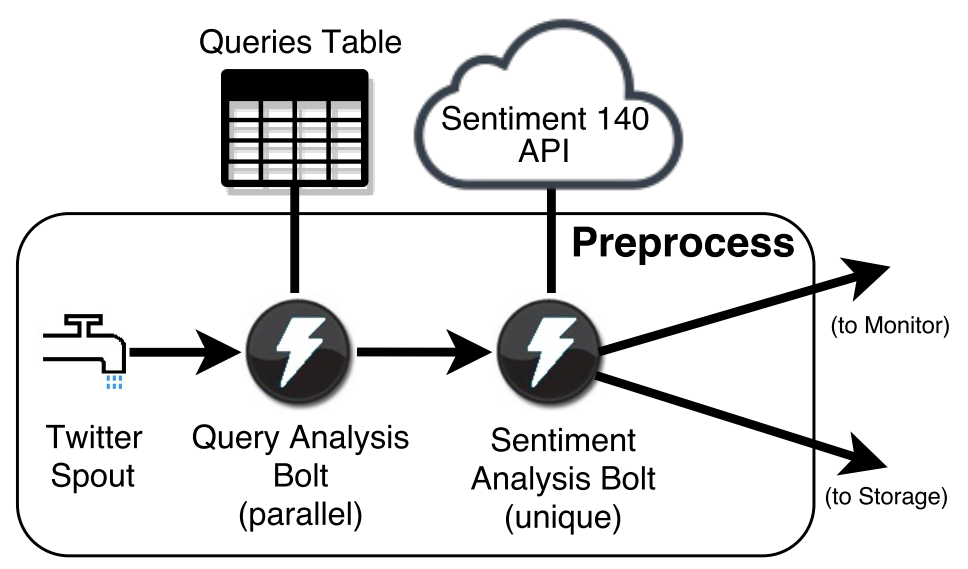

Figure 3.9: Pre-process module in Storm topology, with multistage bolts that implements query and sentiment classification analysis over the stream 


\subsubsection{Real-time Anomaly Detection using TRAD}

The Monitor module operates over the stream that is emitted from the Preprocess module as shown in Figure 3.10. It performs the tasks of aggregation and anomaly detection analysis using the proposed TRAD approach. The input stream from the Preprocess module is partitioned based on the sentiment attribute, generating three streams corresponding to each sentiment. The Aggregation bolt is parallelized with three instances to process these three streams. This means that there will be three threads for the Aggregation bolt, one for each sentiment class. The temporal binning is performed for each query based on the aggregation factor specified by the analyst. The temporal bin count related to each class of sentiment is temporarily stored in memory for each query. The aggregated bins represents a data point in the time series, that are then emitted further to the Anomaly detection bolt.

The Anomaly Detection bolt implements the streaming TRAD algorithm as presented in Algorithm 3.5. The Anomaly Detection bolt is parallelized with three instances, in order to individually process the tweets corresponding to each class of sentiment. The sliding window data structure generated by the TRAD algorithm for each query are stored to an in-memory data structure and has a constant space

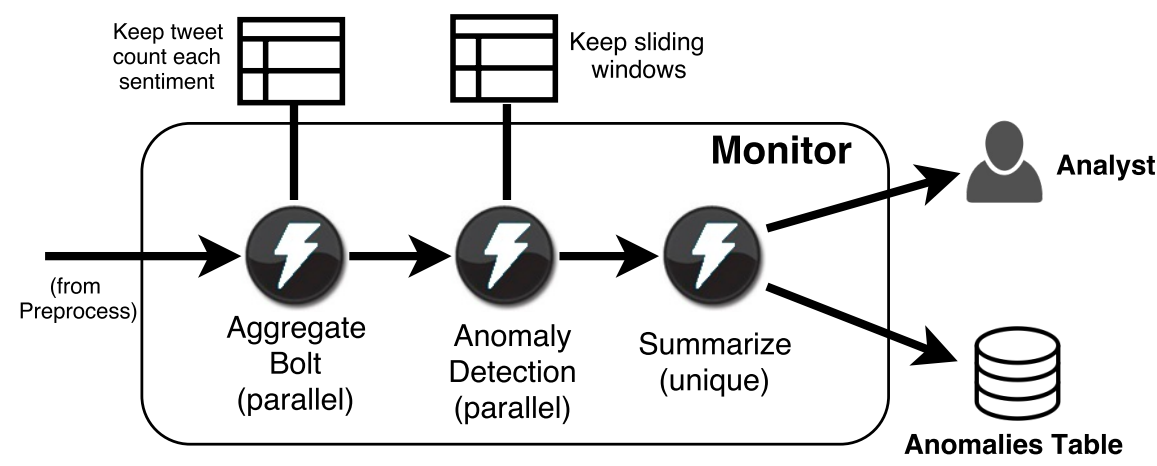

Figure 3.10: The Monitor module in Storm topology, with multistage bolts that implements the aggregation and RSAD approach which operates over the stream provided the from Preprocess module 
requirement. The anomaly detection result emitted by all three instances is summarized by the Summarize bolt, which then further stores the anomaly details in the Anomalies table and notifies the analyst if anomalies are detected. 


\section{Chapter 4}

\section{Evaluation Methodology and Results}

This Chapter presents the experiments performed to compare the four versions of the aforementioned TRAD approach, along with other two anomaly detection techniques discussed specifically in Chapter 2 (SLR and LOF). In Section 4.1, the algorithms that are chosen for comparative experiments are listed with their parameter sets. In Section 4.2, the two datasets that are used for this evaluation are described. Section 4.3 steps through the experimental procedures that were followed and provides the specification of the environment in which the experiments were conducted. Section 4.4 presents the result of the evaluation along with a discussion on the findings.

\subsection{Algorithms}

The primary technique that is under evaluation is the two-stage real-time anomaly detection technique (TRAD), with the four variants for calculating the mean and standard deviation. The two alternative techniques for anomaly detection that are compared with the TRAD technique are Simple Linear Regression (SLR) and Local Outlier Factor (LOF). Interested readers can review the algorithms for TRAD, SLR, 
and LOF techniques in the corresponding Sections 3.3, 2.2.4, and 2.2.5. These techniques are evaluated with the three sentiment classified time series present in each dataset. Each of these techniques operates with different sets of parameters. In the following subsections, these parameters and how they are varied in the experiments are given.

\subsubsection{Two-stage Real-time Anomaly Detection}

In Section 3.2.2, the proposed two-stage real-time anomaly detection (TRAD) technique for anomaly detection is presented with the feasible approaches for detecting the candidate and legitimate anomalies. The combination of the two models that can be applied to detect candidate anomalies (EWMA and PEWMA) and the two models that summarize the statistical properties of the sliding window (STD and MAD) resulted in four variant techniques of the TRAD technique. These are EWMA-STD, EWMA-MAD, PEWMA-STD, and PEWMA-MAD. These techniques are evaluated independently in order to identify the one with comparatively better accuracy in detecting the sentiment-based anomalies that are the focus of this research. The accuracy of each technique is based on its ability to detect true anomalies in the presence of extreme anomalies, and to reject false anomalies in the time series data

streams. In order to evaluate the accuracy of these four techniques for detecting sentiment anomalies in real-world datasets, experiments were performed by varying the following three input parameters in the TRAD algorithm that is presented in Algorithm 3.5:

- $n$ represents the length of the sliding window, which is defined in terms of the number of historic data points to be maintained for detecting the legitimate anomalies. For example, if 1 week of window period is considered with the data aggregation of 1 hour, the value of $n$ will be $n=168$ (24 7 days). The range of values for 
this parameter are different for each dataset and will be defined in the Section corresponding to each dataset.

- The threshold parameter for candidate anomaly detection stage $\tau_{c}$ is varied in the range $[1,5]$ with the step size of 1 .

- The threshold parameter for legitimate anomaly detection stage $\tau_{l}$ is varied in the range $[1,5]$ with the step size of 1 .

The other parameters that were not varied are the decay factor for both EWMA and PEWMA; these are assigned with a fixed value of $\alpha_{E W M A}=0.97$ and $\alpha_{P E W M A}=$ 0.99, respectively. These values for decay factors are the optimal minimum mean square error parameters in many settings [13]. Each of these approaches were implemented in the algorithm by substituting the function calls that summarizes the statistical properties with the appropriate methods for that approach.

\subsubsection{Simple Linear Regression Analysis}

The first alternative technique that was implemented for the experiments is the Simple Linear Regression (SLR) technique. The theory related to the SLR based anomaly detection is discussed in Section 2.2.4. In general, given the input of a list of data points, the output of a regression analysis is a list of error (residual) values. An error value is the difference between the predicted and real value of a data point. In order to detect if an error value is anomalous, extreme value analysis is applied over the list of error values. To evaluate the accuracy of the SLR technique for detecting sentiment anomalies, experiments were performed by varying the following three input parameters in the SLR algorithm discussed in Section 2.2.4:

- The number of historic data points $n$, considered to evaluate the linear regression model in Equation 2.12. The range of values for this parameter are varied in the range $[5,50]$ with step size of 5 . 
- $k$, the length of the sliding window that maintains a list of error values for the historic data points. The list of values that are varied for this parameter are 1, 3, $6,7,10,15,20,30$, and 40 days.

- The threshold parameter $\tau$ for detecting anomalies, which is varied in the range $[1,5]$ with the step size of 1 .

Although the parameters $n$ and $k$ both define the number of historic data points, they are used for different purposes. When performing the linear regression analysis, $n$ data points are considered to generate a linear model. When performing extreme value analysis, the list of error values of size $k$ is considered to generate the normal distribution of the prediction errors.

\subsubsection{Local Outlier Factor}

The second alternative technique that was implemented for the experiments is the Local Outlier Factor (LOF) technique. The theory related to the LOF based anomaly detection is discussed in Section 2.2.5. In general, given the input of a list of data points, the output of the LOF technique is a list of local outlier factor score values. A LOF score value is calculated from Equation 2.18. In order to detect if an LOF score is anomalous, extreme value analysis is applied over the list of LOF score values. To evaluate the accuracy of the LOF technique for detecting sentiment anomalies, experiments were performed by varying the following three input parameters in the LOF algorithm presented in Section 2.2.5:

- The number of neighbours, $N$, taken into consideration for calculating the LOF score. The list of values that are varied for this parameter are 40, 45, 50, 60, and 70 data points.

- The value of the k-nearest neighbour, $k$, considered for calculating the LOF score. The list of values that are varied for this parameter are 20, 25, 30, 35, 40, and 50 
data points, with the constrain that for each value of $N, k$ could not exceed this value.

- The threshold parameter $\tau$, which is varied in the range $[1,5]$ with the step size of 1 .

\subsection{Data Sets}

The two datasets used for the evaluation of the work in this Thesis consist of usergenerated content during multiple real-world events that were widely discussed on social media platforms. The goal here is to be able to detect the sentiment anomalies in the three sentiment classified time series present in these datasets in an automatic way, and with minimal parameter tuning. The two datasets are described in the following subsections.

\subsubsection{Le Tour de France 2013 Dataset}

Le Tour de France is the premier race in professional cycling. The 2013 event was held from June 29 - July 21, 2013 (22 days). The collected dataset contains over 449,077 tweets retrieved from the Twitter public stream that used the official hash tag ("\#tdf") during the event period. This dataset was collected as part of a project that uses visual analytics to discover and analyze the temporally changing sentiment of tweets posted in response to micro-events occurring during a sporting event (such as Le Tour de France) $[26,27]$. The goal for the evaluation of this dataset is to automatically detect these noteworthy micro-events as sentiment anomalies using each candidate technique. Such data is an excellent resource for evaluating the candidate techniques for this Thesis, since users on social media platforms have a propensity for using strong sentiment in their tweets during sport events, they commonly watch 
the event live, and many micro-events occur that may cause anomalous spikes in the number of tweets being posted [26, 27].

It is difficult to readily obtain the classification labels for such user-generated dataset extracted from the Twitter data stream. As this dataset is related to the sport domain, an expert in that domain analyzed these data using the aforementioned visual analytics software to locate the true anomalies. These labels were used to assess the false positives and false negatives identified in the data by each candidate technique. Given the features of this data, the temporal bin length was set to 15 minutes. This dataset was preprocessed using sentiment analysis (Sentiment 140 API [42]) and split into three time series as illustrated in Figure 4.1. The first time series plots the frequency of the tweets with positive sentiment (Figure 4.1 (a)). The second time

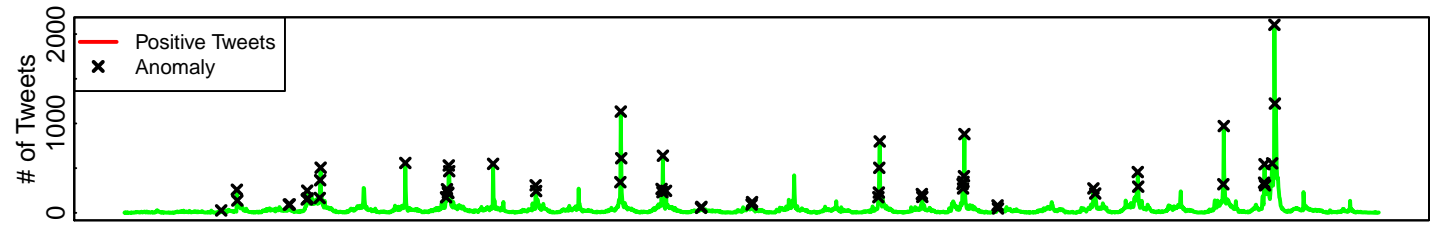

(a) Positive sentiment

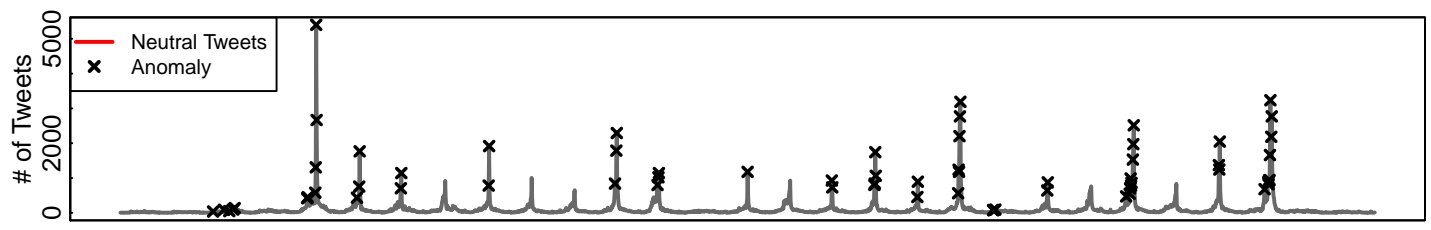

(b) Neutral sentiment

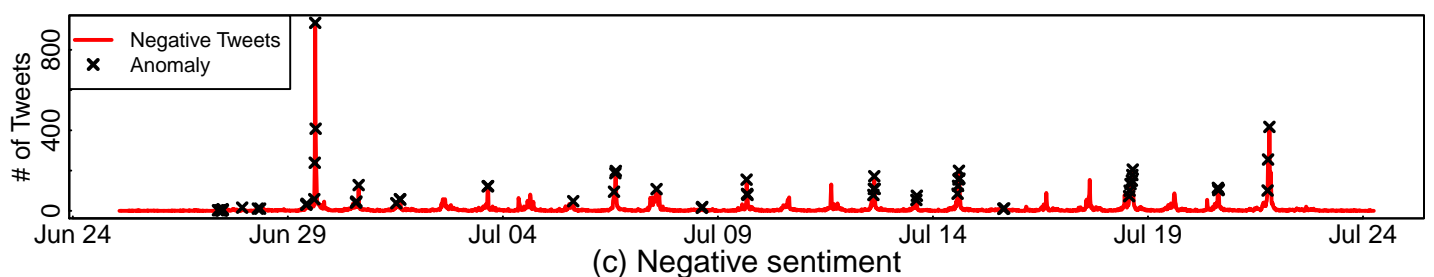

Figure 4.1: Le Tour de France 2013 Dataset, with three time series corresponding to each sentiment. The " $x$ " symbol denotes the true anomalies. Note that for the visibility of the marked anomalies, the scale on Y-axis is adapted for each time series. 
series plots the frequency of the tweets with neutral sentiment (Figure 4.1 (b)). The third time series plots the frequency of the tweets with negative sentiment (Figure $4.1(\mathrm{c}))$.

\subsubsection{The Gavagai Dataset}

The Gavagai dataset was obtained from Andreas et al. [52]. This dataset was provided to them by a company called Gavagai AB. Gavagai AB is located in Stockholm, Sweden, and is involved in research areas related to text analysis of big data. The analysis consists of looking at how many times a topic (e.g., a person, company, or brand) is mentioned in social media platforms as well as regular news websites, and the sentiment used while mentioning the topic. The authors used this dataset in their work to evaluate existing anomaly detection techniques (moving average, simple linear regression, and local outlier factor techniques) with efficacy and efficiency as their primary concern for detecting anomalies in these time series. [52].

The dataset was generated from Gavagai's live environment and the time series data is related to the topic of a Swedish political party called the Social Democrats. This dataset is a good fit to evaluate our work because it was collected the context of user-generated content and consists of three preprocessed time series: positive sentiment, negative sentiment, and total frequency of tweets. The Gavagai dataset has the temporal bin length of 1 hour. Moreover, for each of these time series, the classification labels indicating true anomalies are also present. The key difference in this dataset and the TDF dataset is that instead of the neutral sentiment, the frequency of tweets (which is the total number of tweets per hour) is present as one of the three time series.

Figure 4.2 shows the time series with the labels for true anomalies. The dataset contains information collected between January 1 - September 22, 2014 (261 days). 


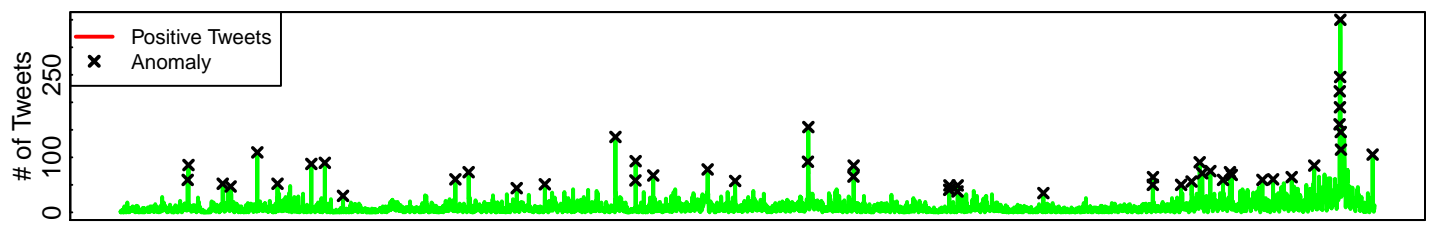

(a) Positive sentiment

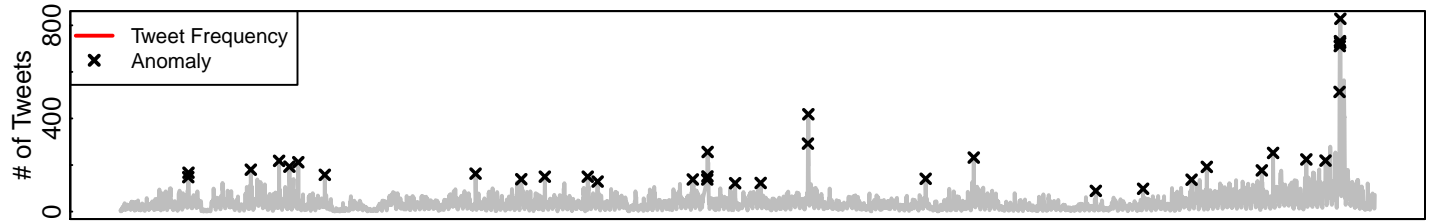

(b) Frequency

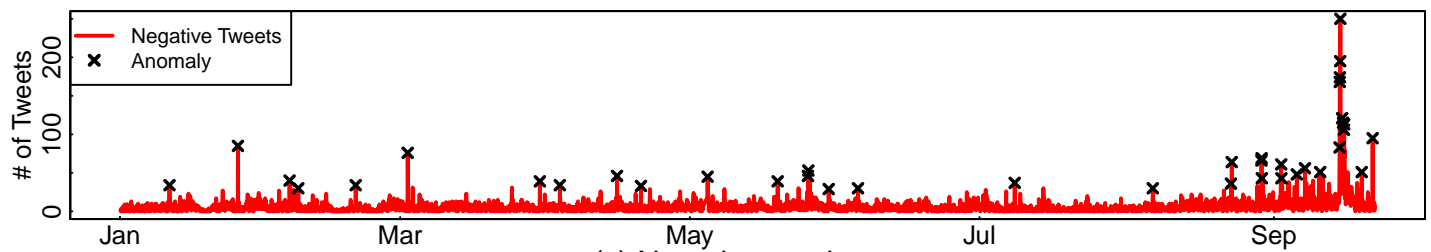

(c) Negative sentiment

Figure 4.2: Gavagai dataset with three timeseries for Social democrats political party. The cross symbol denotes true anomalies. The largest peaks appeared around two elections on May 25 and September 14, 2014.

During this period two popular events occurred: the European Parliamentary Election (May 25th) and the Swedish Parliamentary Election (September 14th). These events can be observed in the dataset presented in Figure 4.2 (a)-(c). In each time series, there is an increase in the frequency of the news near the dates surrounding those two events. Including the above two events, the dataset contains synthetic anomalies that were manually inserted into each time series in this dataset.

\subsubsection{Dataset volatility}

In order to interpret the results presented in this Chapter, the statistical properties of the two datasets are discussed. Volatility is a statistical measure that gives a degree of variation of a time series, and is measured as a standard deviation of a time 
series [1]. A high level of volatility implies that the sentiment changes dramatically over a short period of time. A low level of volatility implies that the sentiment does not fluctuate dramatically, but changes at a steady pace over a period of time. The two datasets that are presented in this Chapter each contain three sentiment-classified time series, which are produced by aggregating the discrete data points from the data streams. The volatility of a time series depends upon the binning interval used for the aggregation. The TDF 2013 dataset has a binning interval of 15 minutes, which was set to allow for a timely identification of anomalies. This dataset has a high level of volatility and the standard deviation of each of the positive, neutral, and negative sentiment time series are 78.77, 216.81, and 25.37 respectively. The Gavagai dataset has a binning interval of 1 hour, which was set to allow for a identification of anomalies over a longer period of time. This dataset has a low level of volatility and the standard deviation of each of the positive sentiment, negative sentiment, and frequency time series are 13.69, 39.68, and 9.19 respectively.

Recall that the definition of an anomaly in Section 3.1 states that a data point is considered anomalous if it deviates sufficiently from nearby data points. The fact that the TDF 2013 dataset has a high level of volatility implies that this dataset has statistically extreme value anomalies. Moreover, the length of this dataset is 22 days and within this period there are 123, 66, and 181 anomalies in the three sentiment classified time series. In contrast, the length of the Gavagai dataset is 261 days, and there are only 85, 36, and 124 anomalies in the three time series datasets. As these datasets has low level as well as high level of volatility in the data, they represent opposite ends of the continuum of bursty user-generated content. Considering the above mentioned statistics in terms of volatility, these two datasets represents an appropriate benchmark, in order to test the performance of the candidate techniques. 


\subsection{Experimental Procedures and Environment}

A parameter set for an algorithm is defined as a set of values corresponding to the parameters in that algorithm. During the evaluation process, each of the candidate algorithms underwent three sequential steps:

1. Reading the input dataset and preprocessing.

2. Executing the algorithm on each time series in the given dataset for a list of parameter sets.

3. Evaluating the performance of the algorithm for each parameter setting in terms of precision, recall, and F-score.

The goal of the evaluation in this Thesis is to discover if there exists a technique with a parameter set that works well across all three sentiment classes. None of the candidate techniques considered for the evaluations are non-parametric. However, for the parametric techniques a parameter set can be identified by tuning the parameters such that the accuracy remains consistent with different time series.

To obtain an optimized parameter set for each technique, it must be tested using a large number of parameter sets. For each of the techniques and for each of the parameter sets, precision and recall were calculated, along with the F-score. Since a high F-score value represents both high precision and recall, we use this as the key measure of effectiveness [3]. An average of these measures were calculated over the positive, neutral, and negative sentiment time series, to identify the parameter set that has better overall accuracy when the three sentiment classes were considered together.

All the candidate algorithms were implemented in Java as part of the Storm framework [4]. The implementation includes the algorithms for the TRAD (four variant), SLR, and LOF techniques, along with the automated evaluation procedure 
to calculate precision, recall, and F-score. The experiments were conducted on a machine configured with an Intel Core i7-3770 processor running at 2.40GHz processor and 12.0 GB of RAM. The three time series for each dataset were stored in an individual comma separated value file (CSV) which contained a list of data points. A data point represented a particular instance in a time series with date, value, and anomaly label. In order to simulate the data stream scenario, a data stream is modelled from these list of data points and then provided as input to the algorithm under evaluation.

\subsection{Results}

Each of the six candidate techniques were evaluated using the two data sets, as described in Sections 4.1 and 4.2, resulting in total of 12 evaluations that are presented in this section. Each evaluation was run multiple times with a different configuration from the parameter sets. The results are illustrated in a single figure and corresponding table, as described below:

1. The figure contains four graphs representing the F-score information on the $\mathrm{x}$-axis

and the key parameter from the parameter set on y-axis for each of the sentiment classes: positive, neutral, and negative along with the average ((positive + neutral + negative)/3).

2. The table lists the parameter set that resulted in the highest F-score for each of the sentiment classes and the average.

The two alternative techniques SLR and LOF are optimized to analyze data streams by considering the sliding window of data instead of full dataset. With this optimization the execution time of these techniques reduced significantly over both the datasets. As all of the techniques under evaluation including TRAD (Section 3.4) 
have runtimes far less than 1 second per data point, we have chosen not to explicitly state the runtimes in this evaluation.

In the rest of this section, the results are presented for each of the two datasets independently. For a given dataset the results are organized as follows. First, the results from the evaluations conducted for the four variants of the TRAD technique are presented and the approach with the best F-score is identified. Second, the evaluation for the SLR technique is presented and compared with the best variant of the TRAD technique. Third, the evaluation for the LOF technique is presented and compared with the best variant of the TRAD technique. At last, the top three techniques are identified among the six candidate techniques whose average F-score are the highest across both test datasets.

\subsubsection{Le Tour de France 2013 Dataset}

Two-stage Real-time Anomaly Detection (TRAD): As described in Section 4.1.1, the three parameters that were varied for all of the four candidates of TRAD technique are $n$ (window size), $\tau_{c}$, and $\tau_{l}$. The values of $n$ were $1,3,6,7,10,15,20$, 30, and 40 days, where the last value (40 days) represents a non-sliding window as that is the maximum length of the dataset. The evaluation of the TRAD technique using this dataset involved total of 225 parameter sets.

First, the optimal value for $n$ was independently evaluated, and was set to 6 days for this dataset. The results of the evaluation conducted for identifying the best window size is given in Appendix Table A.1. Second, once the optimal window size was determined, the F-score results were generated by varying the $\tau_{c}$ and $\tau_{l}$ parameters. An optimal parameter setting for a time series is the one with the highest F-score. The results are presented in Figures 4.3 - 4.6 and Tables 4.1 - 4.4. 


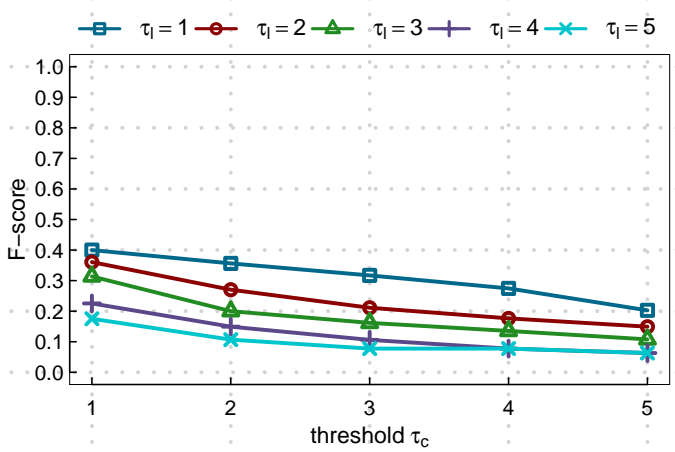

(a) Positive sentiment

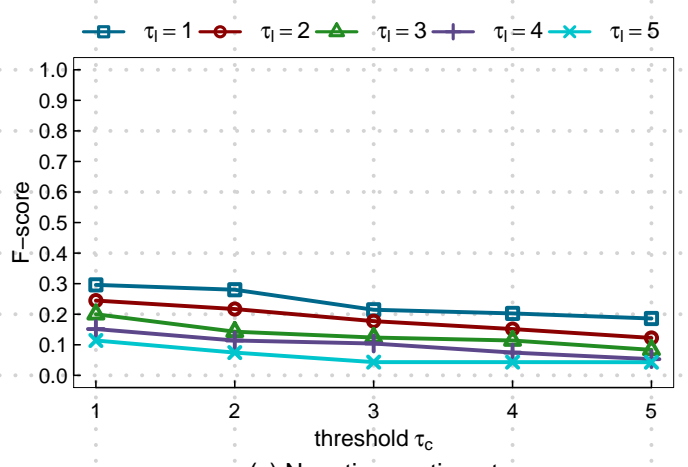

(c) Negative sentiment

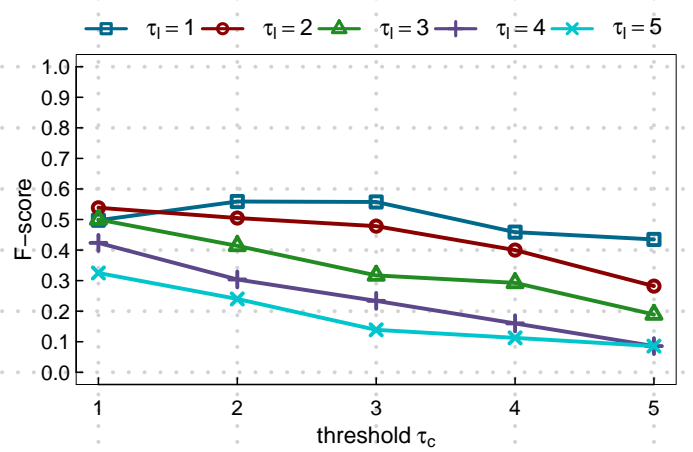

(b) Neutral sentiment

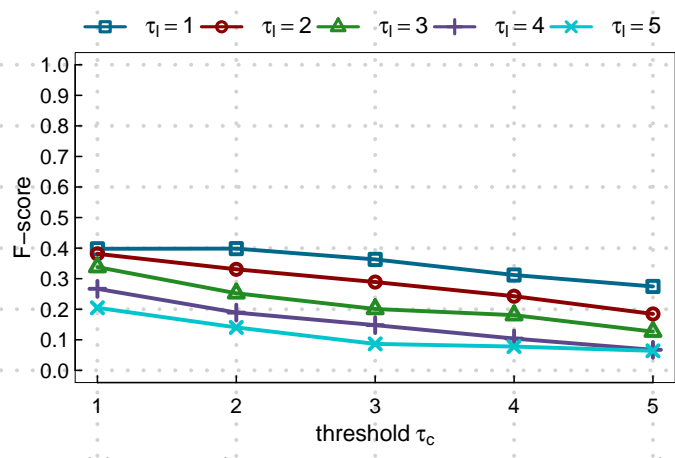

(d) Average (Postive + Neutral + Negative sentiment)

Figure 4.3: F-score results for the EWMA-STD approach with the TDF dataset

Table 4.1: Optimal parameter sets for the EWMA-STD approach with the TDF dataset

\begin{tabular}{|l|c|c|c|c|}
\hline Sentiment & Parameters & Precision & Recall & F-score \\
\hline Positive & $\tau_{c}=1, \tau_{l}=1$ & 0.401 & 0.398 & 0.400 \\
\hline Neutral & $\tau_{c}=2, \tau_{l}=1$ & 0.542 & 0.575 & 0.558 \\
\hline Negative & $\tau_{c}=1, \tau_{l}=1$ & 0.365 & 0.248 & 0.296 \\
\hline Average & $\tau_{c}=2, \tau_{l}=1$ & 0.541 & 0.342 & 0.398 \\
\hline
\end{tabular}

As depicted in Figure 4.3, the F-score for the EWMA-STD approach generally decreases with increases in $\tau_{c}$ and $\tau_{l}$. However, the F-score rises slightly for $\tau_{c}=$ 2 and $\tau_{l}=1$, particularly in the results for the neutral sentiment and the average. Table 4.1 shows that these were the optimal parameter set for the neutral sentiment, and sufficiently good to produce the best F-score when averaging the results. 


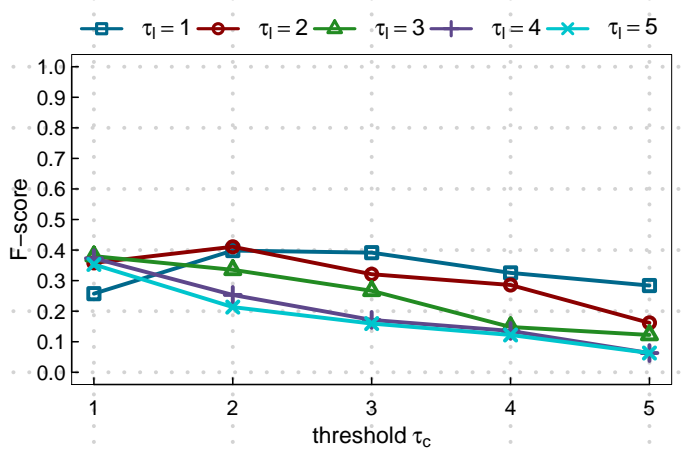

(a) Positive sentiment

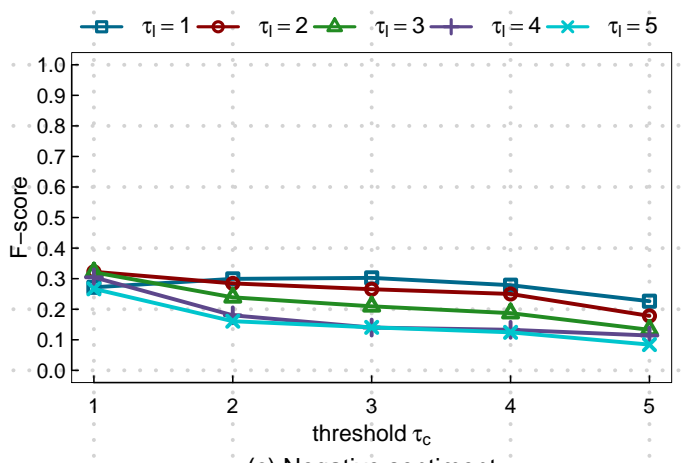

(c) Negative sentiment

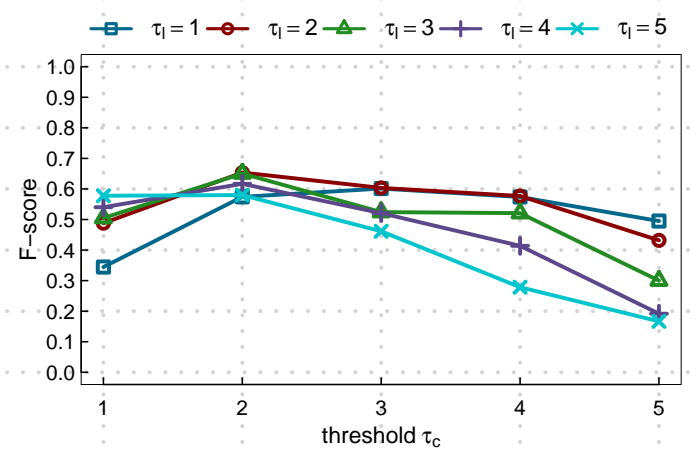

(b) Neutral sentiment

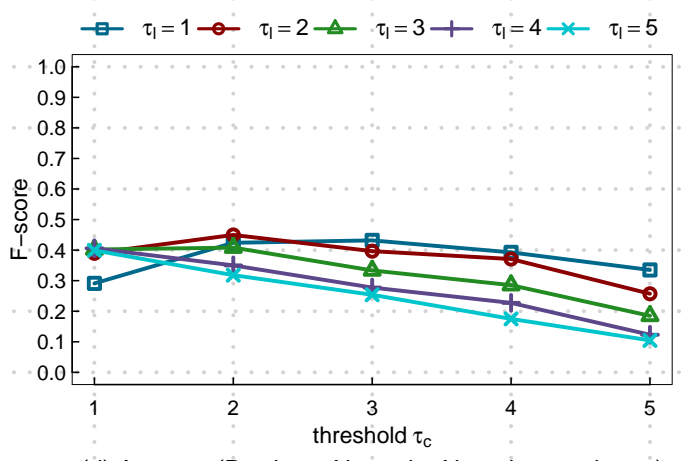

(d) Average (Postive + Neutral + Negative sentiment)

Figure 4.4: F-score results for the EWMA-MAD approach with the TDF dataset

Table 4.2: Optimal parameter sets for the EWMA-MAD approach with the TDF dataset

\begin{tabular}{|l|c|c|c|c|}
\hline Sentiment & Parameters & Precision & Recall & F-score \\
\hline Positive & $\tau_{c}=2, \tau_{l}=2$ & 0.612 & 0.308 & 0.410 \\
\hline Neutral & $\tau_{c}=2, \tau_{l}=2$ & 0.574 & 0.757 & 0.653 \\
\hline Negative & $\tau_{c}=1, \tau_{l}=2$ & 0.387 & 0.276 & 0.322 \\
\hline Average & $\tau_{c}=2, \tau_{l}=2$ & 0.575 & 0.419 & 0.449 \\
\hline
\end{tabular}

Figure 4.4 shows that the F-score for the EWMA-MAD approach generally decreases with increase in $\tau_{c}$ and $\tau_{l}$, after $\tau_{c}=2$. As shown in Table 4.2, for this approach the highest F-score achieved was for the neutral sentiment with the parameter set $\tau_{c}=2$ and $\tau_{l}=2$. This parameter set also resulted in the best results for the positive and average cases. 


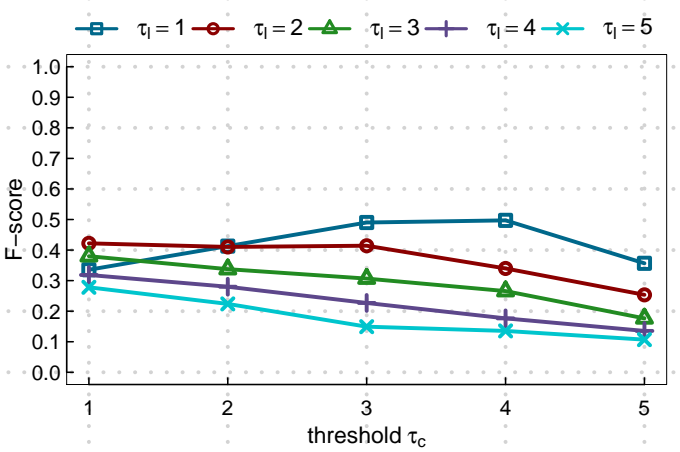

(a) Positive sentiment

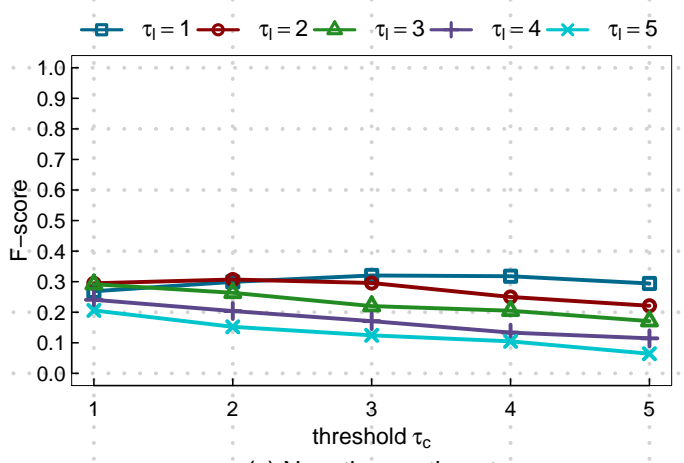

(c) Negative sentiment

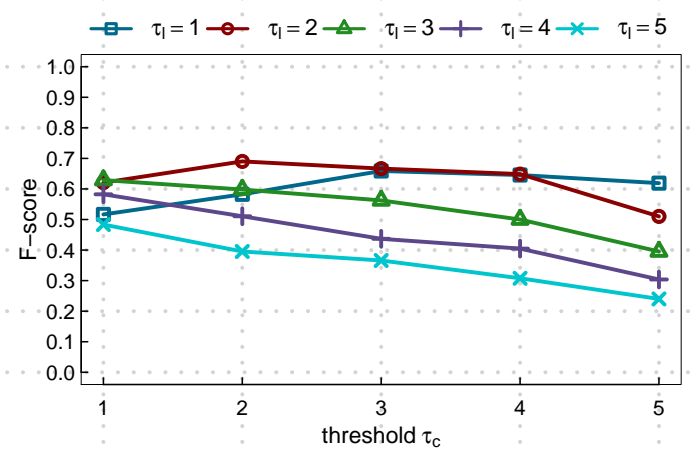

(b) Neutral sentiment

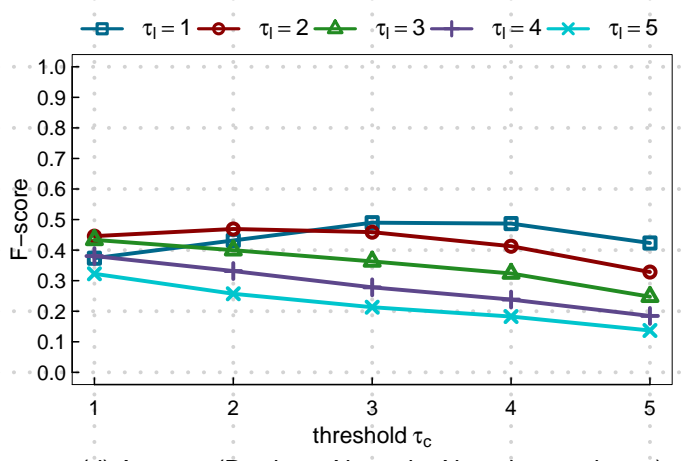

(d) Average (Postive + Neutral + Negative sentiment)

Figure 4.5: F-score results for the PEWMA-STD approach with the TDF dataset

Table 4.3: Optimal parameter sets for the PEWMA-STD approach with the TDF dataset

\begin{tabular}{|l|c|c|c|c|}
\hline Sentiment & Parameters & Precision & Recall & F-score \\
\hline Positive & $\tau_{c}=4, \tau_{l}=1$ & 0.814 & 0.357 & 0.497 \\
\hline Neutral & $\tau_{c}=2, \tau_{l}=2$ & 0.644 & 0.742 & 0.690 \\
\hline Negative & $\tau_{c}=3, \tau_{l}=1$ & 0.450 & 0.248 & 0.320 \\
\hline Average & $\tau_{c}=3, \tau_{l}=1$ & 0.535 & 0.501 & 0.489 \\
\hline
\end{tabular}

As depicted in Figure 4.5, the F-score for the PEWMA-STD approach generally decreases with $\tau_{l}$, but increased then decreased with $\tau_{c}$. As shown in Table 4.3, for this approach the highest F-score achieved was for the neutral sentiment with the parameter set $\tau_{c}=2$ and $\tau_{l}=2$. However, when averaging the results, the highest F-score was for the parameter set $\tau_{c}=3$ and $\tau_{l}=1$. 


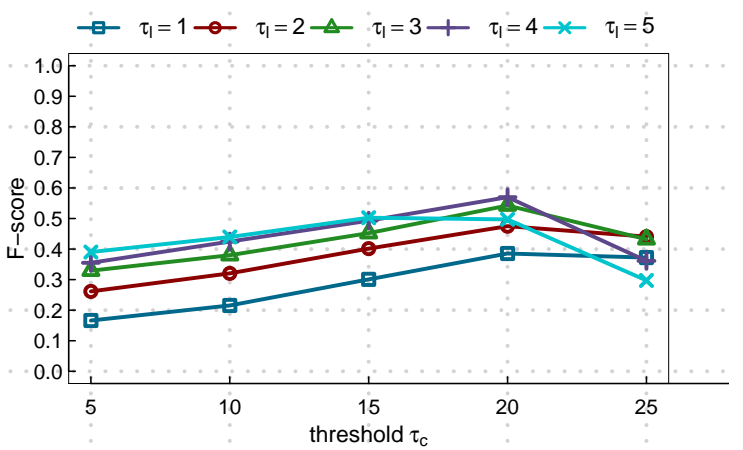

(a) Positive sentiment

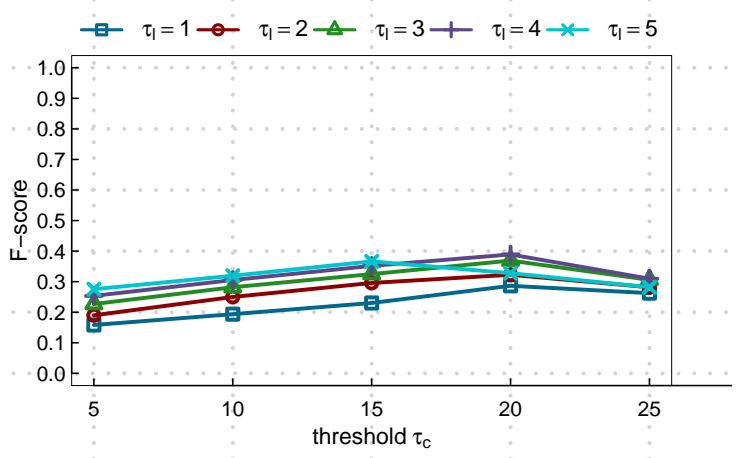

(c) Negative sentiment

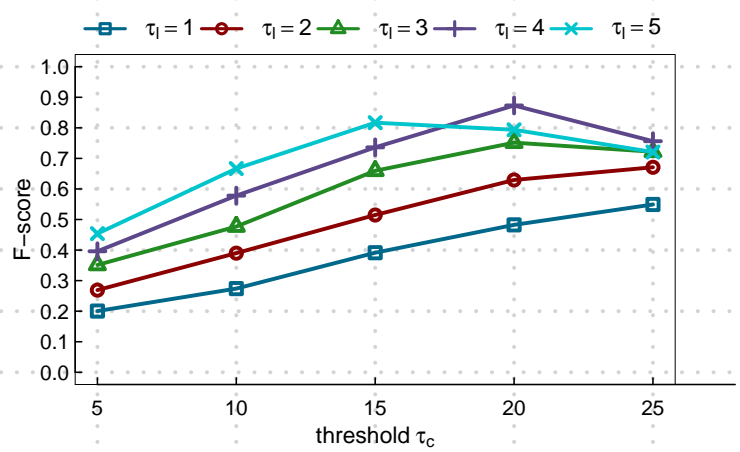

(b) Neutral sentiment

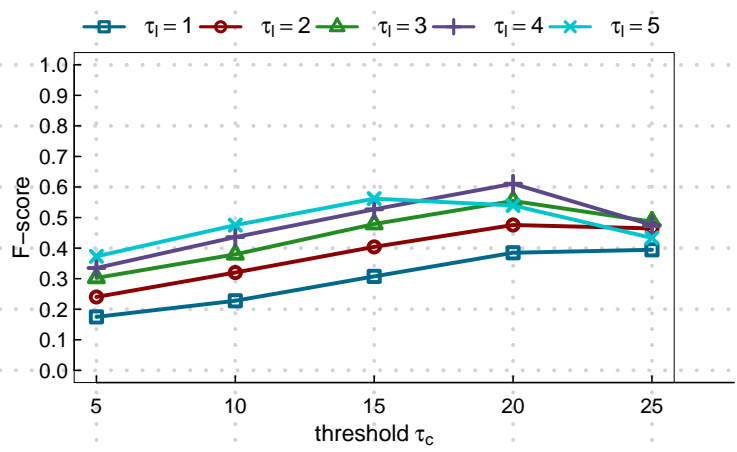

(d) Average (Postive + Neutral + Negative sentiment)

Figure 4.6: F-score results for the PEWMA-MAD approach with the TDF dataset

Table 4.4: Optimal parameter sets for the PEWMA-MAD approach with the TDF dataset

\begin{tabular}{|l|c|c|c|c|}
\hline Sentiment & Parameters & Precision & Recall & F-score \\
\hline Positive & $\tau_{c}=4, \tau_{l}=4$ & 0.910 & 0.414 & 0.569 \\
\hline Neutral & $\tau_{c}=4, \tau_{l}=4$ & 0.815 & 0.939 & 0.873 \\
\hline Negative & $\tau_{c}=4, \tau_{l}=4$ & 0.657 & 0.276 & 0.389 \\
\hline Average & $\tau_{c}=4, \tau_{l}=4$ & 0.794 & 0.543 & 0.610 \\
\hline
\end{tabular}

Contrary to the other approaches, Figure 4.6 shows that, the F-score for the PEWMA-MAD approach increases with increase in $\tau_{c}$ and $\tau_{l}$ to maximum when at $\tau_{c}=4$ and $\tau_{l}=4$. As shown in Table 4.4, for this approach the highest F-score achieved over all sentiment classes with parameter set $\tau_{c}=4$ and $\tau_{l}=4$. 
The following discussion is given in order to identify the best variant approach for the TRAD technique in the context of the TDF 2013 dataset. First, the comparative study of the behavior of the EWMA and PEWMA is presented in relation to the parameter $\tau_{c}$. Looking at the results for the approaches that use EWMA for the candidate anomaly detection stage (i.e., EWMA-STD (Figure 4.3) and EWMA-MAD (Figure 4.4)), one can see that there is an inverse relation between the F-score and the parameter $\tau_{c}$. As this parameter is increased the method for determining if a data point is considered a candidate anomaly will be more strict resulting in a decrease in the F-score. This is because with the increase in $\tau_{c}$, many anomalies were not detected negatively affecting the accuracy.

The results for the approaches that use PEWMA for the candidate anomaly detection stage (i.e., PEWMA-STD (Figure 4.5) and PEWMA-MAD (Figure 4.6)), show that the relation between the F-score and parameter $\tau_{c}$, in case of the PEWMA-STD approach there was a direct relation $\left(\tau_{l}=\{1\}\right)$ and inverse relation $\left(\tau_{l}=\{2,3,4,5\}\right)$, whereas for the PEWMA-MAD approach there was a direct relation for all the values of $\tau_{l}$. The direct relation was present because for the small value of $\tau_{c}$ the precision was high (those that met this criteria were clearly anomalies) and the recall was adversely affected with many actual anomalies not being detected, whereas for the large value of $\tau_{c}$ the precision and recall both increased. This is because for the large values of $\tau_{c}$, PEWMA was able to detect the true extreme anomalies presented in this dataset with high volatility, avoiding the noise in the local context.

Comparing the performance of the EWMA and PEWMA, as discussed in Section 3.2.2, EWMA was affected by the extreme anomalies. This resulted in significant distortion in the mean value from its true representation, making it difficult to discover additional anomalies in the local context as the threshold value increases. However, PEWMA was resilient to extreme anomalies and was able to represent the mean 
close to its true representation. As a result, the PEWMA approach produced a high precision and recall contributing to high F-score value. Thus, as compared to EWMA, PEWMA was able detect the true candidate anomalies with higher accuracy; it is concluded that PEWMA is better than EWMA for this dataset.

Second, in the context of the PEWMA a comparative study of the behaviour of the STD and MAD is presented in relation to the parameter $\tau_{l}$. Looking at the results for the PEWMA-STD approach (Figure 4.5), it shows that there was an inverse relation between the F-score and parameter $\tau_{l}$. Here the STD is only calculated over the data points present in the sliding window. As the number of data points were comparatively small, the results shows that the STD was extremely sensitive to the change in $\tau_{l}$ value. As the parameter $\tau_{l}$ was increased, the method for determining if a candidate anomaly is considered a legitimate anomaly became more strict, resulting in a decrease in the F-score. While this resulted in high precision (those that met this criteria were clearly anomalies), the recall was adversely affected with many actual anomalies not being detected.

The results for the PEWMA-MAD approach (Figure 4.6) shows that there was a direct relation between the F-score and parameter $\tau_{l}$. MAD is also calculated over the data points present in the sliding window. Even though the number of data points were small, the results show that MAD was not particularly sensitive to the change in $\tau_{l}$ value. This is because, as discussed in Section 3.2.2, MAD is immune to the size of data, especially when the size is small it is able to provide a better measure of central tendency of the data considered.

Comparing the performance of the STD and MAD, as discussed in Section 3.2.2 and Section 3.2.1, within the sliding window, there are comparatively fewer data points. If these data points have extreme values, then the simple average that is used to calculate STD fails to represent the central tendency of the window. Considering 
Table 4.5: Average F-score results summarized for all the four variants of TRAD technique with the TDF dataset (Maximum values are bold)

\begin{tabular}{|l|c|c|c|c|}
\hline Sentiment & $\begin{array}{c}\text { EWMA- } \\
\text { STD }\end{array}$ & $\begin{array}{c}\text { EWMA- } \\
\text { MAD }\end{array}$ & $\begin{array}{c}\text { PEWMA- } \\
\text { STD }\end{array}$ & $\begin{array}{c}\text { PEWMA- } \\
\text { MAD }\end{array}$ \\
\hline Positive & 0.400 & 0.410 & 0.497 & $\mathbf{0 . 5 6 9}$ \\
\hline Neutral & 0.558 & 0.653 & 0.690 & $\mathbf{0 . 8 7 3}$ \\
\hline Negative & 0.296 & 0.322 & 0.320 & $\mathbf{0 . 3 8 9}$ \\
\hline Average & 0.398 & 0.449 & 0.489 & $\mathbf{0 . 6 1 0}$ \\
\hline
\end{tabular}

MAD, the median that is used to calculate MAD was able to represent the mean close to the central tendency for the window. Thus, from these results it is inferred that MAD is able to detect the true legitimate anomalies with higher accuracy as compared to the STD.

Table 4.5 shows that the PEWMA-MAD approach is superior to the other variants discussed in the TRAD technique across all sentiment classes and the average. Considering every class of sentiment and the average of them, the PEWMA-MAD approach achieved highest average F-score of $0.610\left(\tau_{c}=4, \tau_{l}=4\right)$.

Simple Linear Regression Approach: As described in Section 4.1.2, the three parameters that were varied for the SLR technique are $n$ (number of data point for calculating the linear regression), $k$ (length of a sliding window for extreme value analysis), and $\tau$ (threshold for extreme value analysis). The values of $k$ were 1,3 , $6,7,10,15,20,30$, and 40 days, where the last value (40 days) represents a nonsliding window as that is the maximum length of the dataset. The evaluation of SLR technique using this dataset involved experimenting with a total of 450 parameter sets.

First, the optimal value for $k$ was independently evaluated and it was set to 6 days for this dataset. The results of the evaluation conducted for identifying the best window size is given in Appendix Table A.2. Once the optimal value for $k$ was set, 
the experiments were conducted by varying the $n$ and $\tau$ parameters. The results are presented in Figure 4.7. An optimal parameter setting for each of the three sentiment classes and the average of them is presented in Table 4.6.

Looking at the results for the SLR technique in Figure 4.7, there is a pattern of the F-score increasing as the value of $\tau$ increases, which is similar to the pattern seen with PEWMA-MAD. As this parameter is increased the method for determining if a

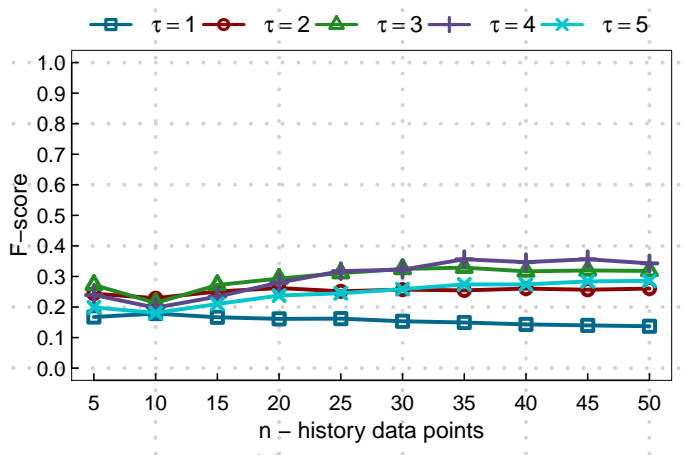

(a) Positive sentiment

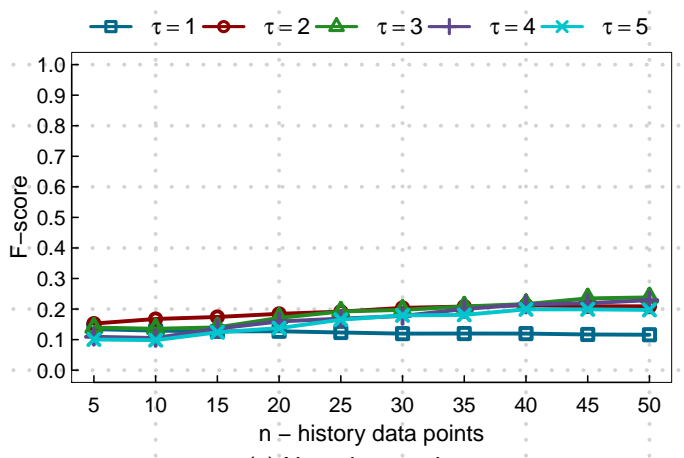

(c) Negative sentiment

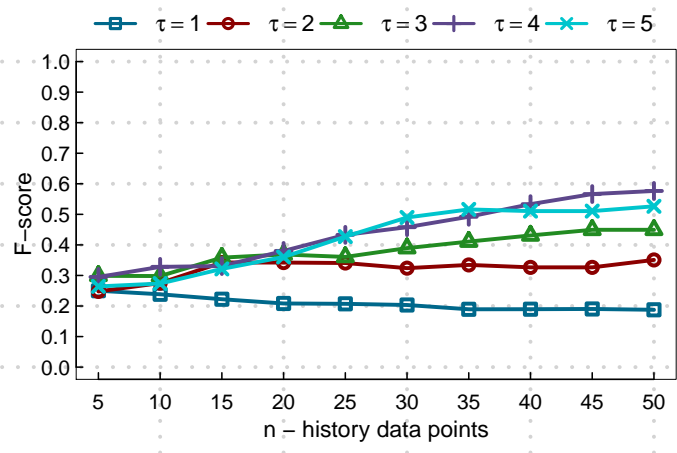

(b) Neutral sentiment

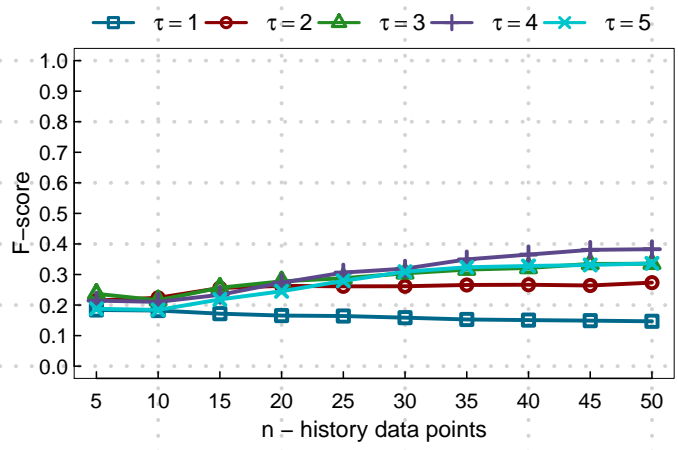

(d) Average (Postive + Neutral + Negative sentiment)

Figure 4.7: F-score results for the SLR approach with the TDF dataset

Table 4.6: Optimal parameter sets for the SLR approach with the TDF dataset

\begin{tabular}{|l|c|c|c|c|}
\hline Sentiment & Parameters & Precision & Recall & F-score \\
\hline Positive & $n=35, \tau=4$ & 0.607 & 0.252 & 0.356 \\
\hline Neutral & $n=50, \tau=4$ & 0.711 & 0.484 & 0.576 \\
\hline Negative & $n=50, \tau=3$ & 0.309 & 0.193 & 0.238 \\
\hline Average & $n=50, \tau=4$ & 0.617 & 0.290 & 0.382 \\
\hline
\end{tabular}


data point is considered an anomaly will be more at ease resulting in an increase in the F-score. Moreover, there is a gradual increase in the F-score with the increase in the value of $n(n=50, \tau=4)$, which flattens out at $n=50$.

Comparing SLR explicitly to PEWMA-MAD, the former approach performed rather poorly with the F-score of $0.382(n=50, \tau=4)$. Recall that PEWMA-MAD achieved its highest average F-score of 0.610. This is because the SLR technique is based on predictive analysis and performs well only for detecting rare extreme peaks. When an extreme anomalous peak is detected, the SLR approach has difficulty detecting comparatively lower anomalous peaks. While this resulted in high precision (those that met this criteria were clearly anomalies), the recall was adversely affected with many actual anomalies not being detected.

Local Outlier Factor Approach: As described in Section 4.1.3, the three parameters that were varied for LOF technique are $N$ (number of neighbours considered), $k$ ( $k$-nearest neighbour), and $\tau$ (threshold for extreme value analysis). The values of $N$ were $40,45,50,60$, and 70 data points. The values of $k$ were $20,25,30,35,40$, and 50 data points with the constraints that for each value of $\mathrm{N}, k$ could not exceed this value. The evaluation of LOF technique using this dataset involved a total of 450 different parameter sets.

First, the optimal value for $N$ was independently evaluated and it was set to 70 neighbour data points for this dataset. The results of the evaluation conducted for identifying the best window size is given in Appendix Table A.3. Once the optimal value for $N$ was set, the experiments were conducted by varying the $k$ and $\tau$ parameters. The results are presented in Figure 4.8. An optimal parameter setting for each of the three sentiment classes and the average of them is presented in Table 4.7.

Looking at the results for the local outlier factor technique in Figure 4.8, it shows that the F-score increases until the middle values of $\tau$ and $k$, and then it decreases. As 


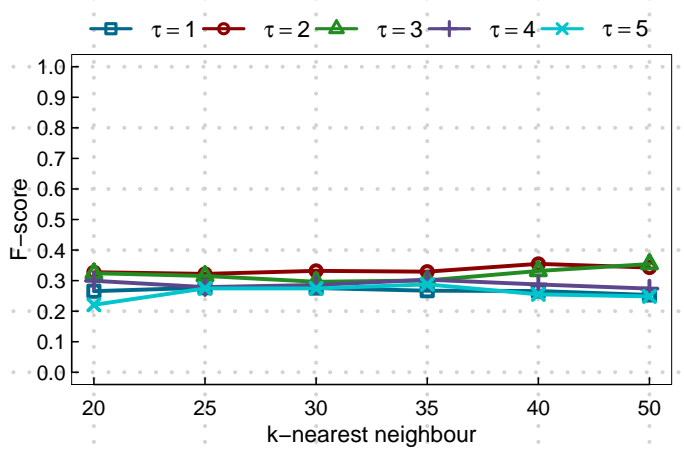

(a) Positive sentiment

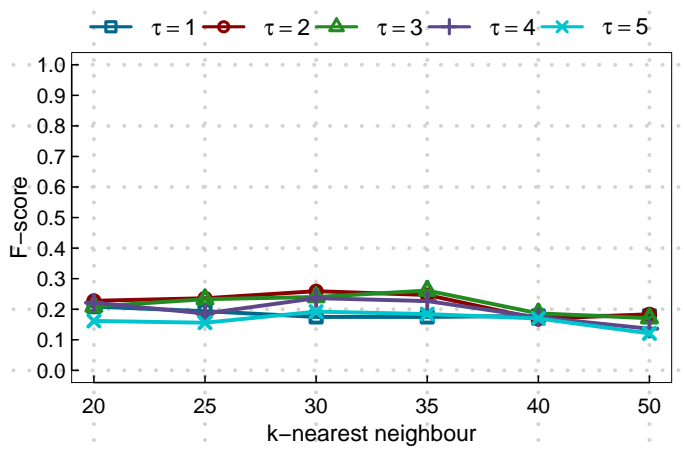

(c) Negative sentiment

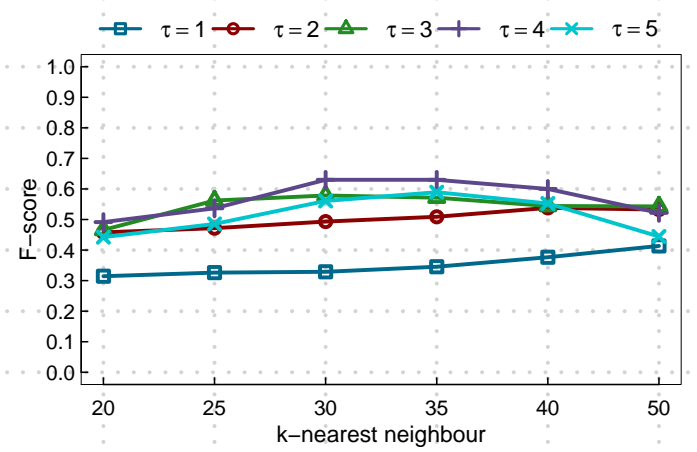

(b) Neutral sentiment

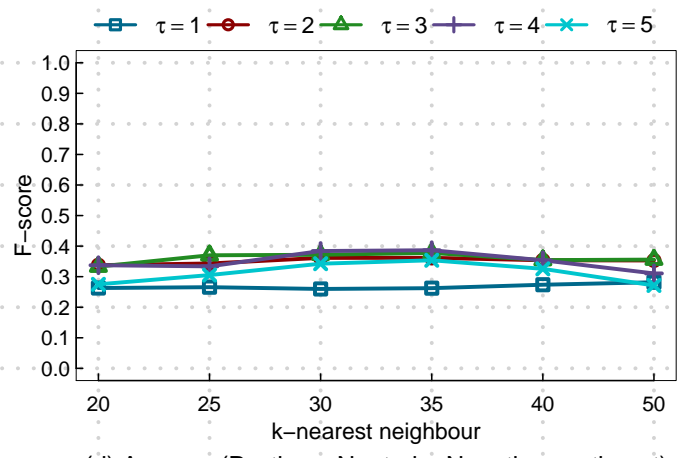

(d) Average (Postive + Neutral + Negative sentiment)

Figure 4.8: F-score results for the LOF approach with the TDF dataset

Table 4.7: Optimal parameter sets for the LOF approach with the TDF dataset

\begin{tabular}{|l|c|c|c|c|}
\hline Sentiment & Parameters & Precision & Recall & F-score \\
\hline Positive & $k=40, \tau=2$ & 0.352 & 0.357 & 0.354 \\
\hline Neutral & $k=30, \tau=4$ & 0.655 & 0.606 & 0.629 \\
\hline Negative & $k=35, \tau=3$ & 0.345 & 0.209 & 0.261 \\
\hline Average & $k=35, \tau=4$ & 0.558 & 0.321 & 0.386 \\
\hline
\end{tabular}

shown in Table 4.7, for this approach the highest F-score achieved was for the neutral sentiment with the parameter setting $k=30, \tau=4$. However, when averaging the results, the highest F-score was for the parameter setting $k=35, \tau=4$.

However, when the results of the LOF technique are compared with the SLR technique in Table 4.8 the performance of the LOF technique is marginally better with 0.386 as the highest average F-score, where as the SLR technique achieved 
Table 4.8: Average F-score results summarized for PEWMA-MAD, SLR, and LOF methods with the TDF dataset

\begin{tabular}{|c|c|c|c|}
\hline Sentiment & $\begin{array}{c}\text { PEWMA- } \\
\text { MAD }\end{array}$ & SLR & LOF \\
\hline Positive & $\mathbf{0 . 5 6 9}$ & 0.356 & 0.354 \\
\hline Neutral & $\mathbf{0 . 8 7 3}$ & 0.576 & 0.629 \\
\hline Negative & $\mathbf{0 . 3 8 9}$ & 0.238 & 0.261 \\
\hline Average & $\mathbf{0 . 6 1 0}$ & 0.382 & 0.386 \\
\hline
\end{tabular}

only 0.382. Moreover, comparing LOF to the PEWMA-MAD approach, the former approach performed poorly with the F-score of 0.386 , which is much lower than the F-score achieved by the PEWMA-MAD approach (0.610).

Discussion: From Table 4.8, it can be conclude that for the Le Tour de France dataset, the PEWMA-MAD approach achieved the highest average F-score of 0.610 as compared with all the other candidate methods. While the other techniques approached this value for certain parameter sets, the average was nowhere near what was achieved by PEWMA-MAD. Given the resilience of PEWMA-MAD to the threshold parameters, we conclude that it is the superior approach among the other approaches for finding anomalies in the Le Tour de France 2013 dataset. 


\subsubsection{The Gavagai Dataset}

In this subsection the results for six candidate techniques that were evaluated using the Gavagai dataset are presented, with the concise discussion for the result of each technique. For each candidate technique, the same parameters from the previous data set were used, with the exception of the ones that are dependent on the length of this dataset.

Two-stage Real-time Anomaly Detection (TRAD): The values of $n$ for this dataset were in the range $[5,263]$ days with the step length of 10 days, where the last value (263) represents a non-sliding window. The evaluation of the TRAD technique using this dataset involved total of 650 different parameter sets.

First, the optimal value for $n$ (window size) was independently evaluated and it was set to 21 days for this data set. The results of the evaluation conducted for identifying the optimal window size are given in Appendix Table B.1. Once the optimal window size was evaluated, the F-score results were generated by varying the $\tau_{c}$ and $\tau_{l}$ parameters. The results are presented in Figure 4.9 - 4.12. An optimal parameter setting for each of the three sentiment classes and the average of them are presented in Table 4.9 - Table 4.12 . 


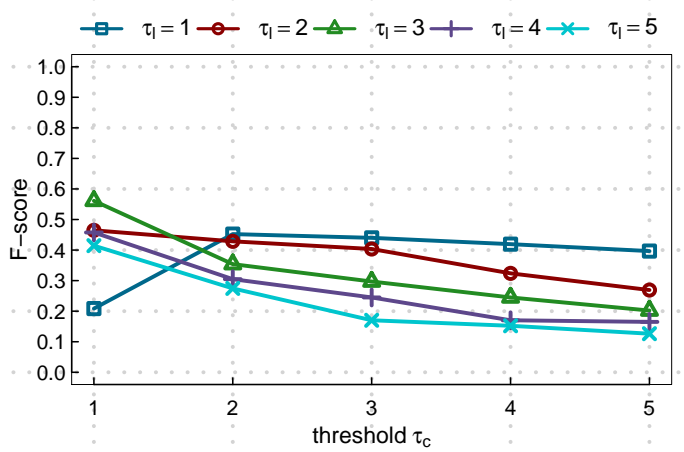

(a) Positive sentiment

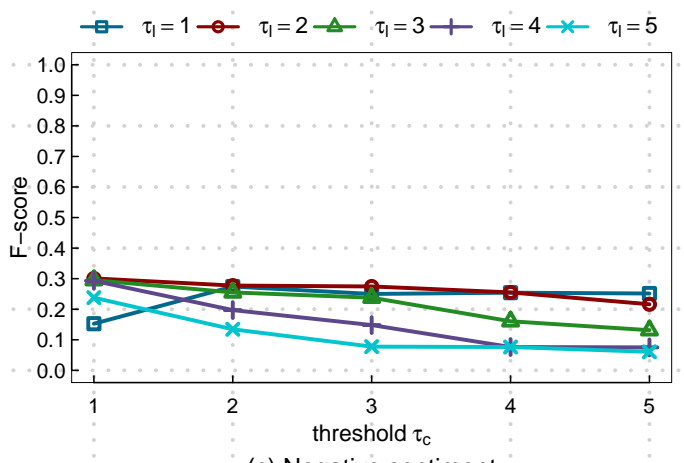

(c) Negative sentiment

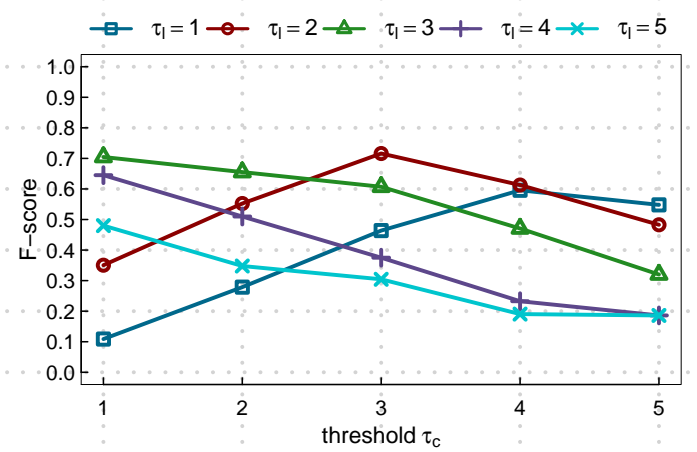

(b) Frequency

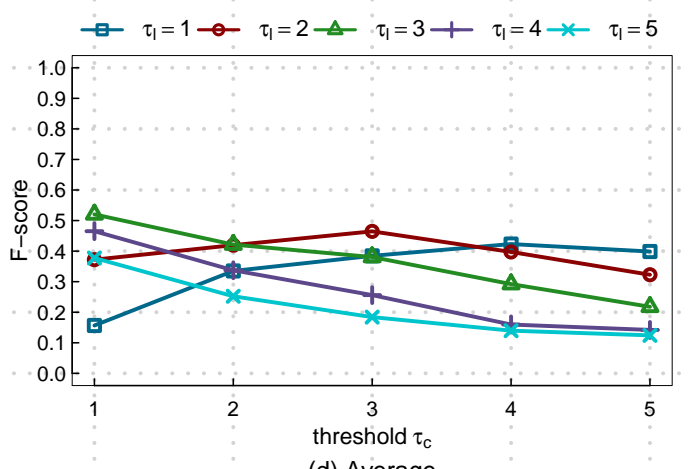

(d) Average

Figure 4.9: F-score results for the EWMA-STD approach with the Gavagai dataset

Table 4.9: Optimal parameter sets for the EWMA-STD approach with the Gavagai dataset

\begin{tabular}{|l|c|c|c|c|}
\hline Sentiment & Parameters & Precision & Recall & F-score \\
\hline Positive & $\tau_{c}=1, \tau_{l}=3$ & 0.722 & 0.458 & 0.561 \\
\hline Frequency & $\tau_{c}=3, \tau_{l}=2$ & 0.644 & 0.805 & 0.716 \\
\hline Neutral & $\tau_{c}=1, \tau_{l}=2$ & 0.303 & 0.298 & 0.300 \\
\hline Average & $\tau_{c}=1, \tau_{l}=3$ & 0.592 & 0.512 & 0.520 \\
\hline
\end{tabular}

As shown in Figure 4.9, the F-score for the EWMA-STD approach increases with increase in $\tau_{c}$ until it reaches a value of 3 , and then generally decreases with increase with $\tau_{c}$. As shown in Table 4.9, for this approach the highest F-score achieved was for the neutral sentiment with the parameter set $\tau_{c}=3$ and $\tau_{l}=2$. However, this approach produced the highest average F-score for parameter set $\tau_{c}=1$ and $\tau_{l}=3$. 


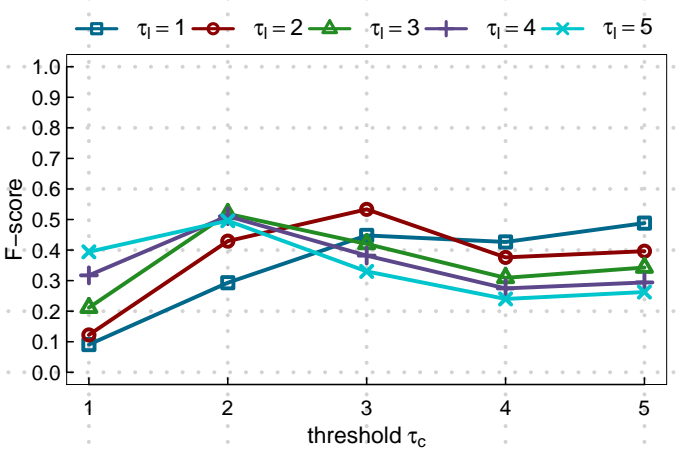

(a) Positive sentiment

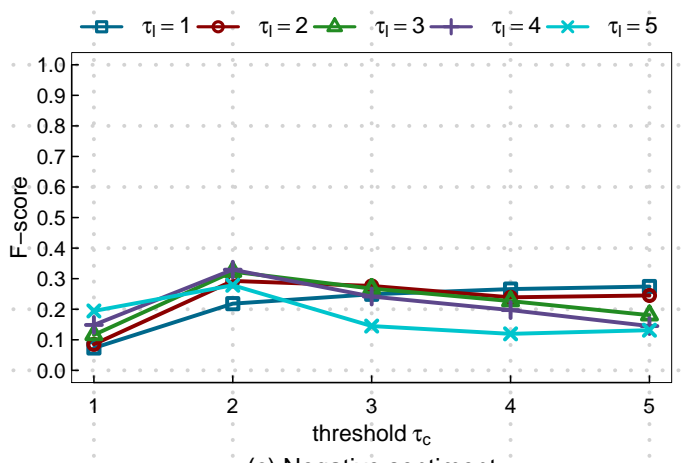

(c) Negative sentiment

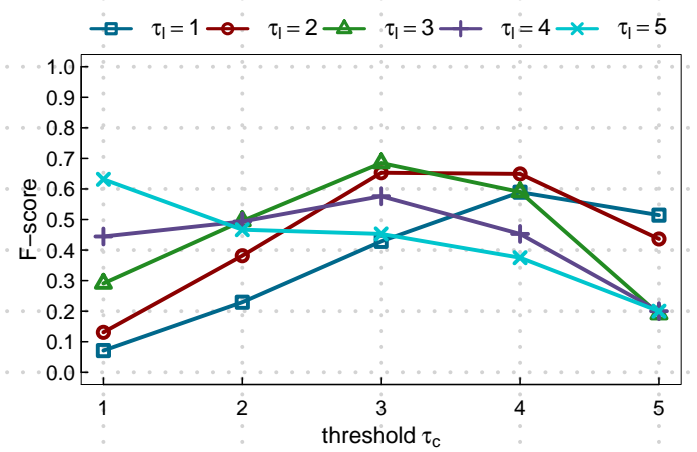

(b) Frequency

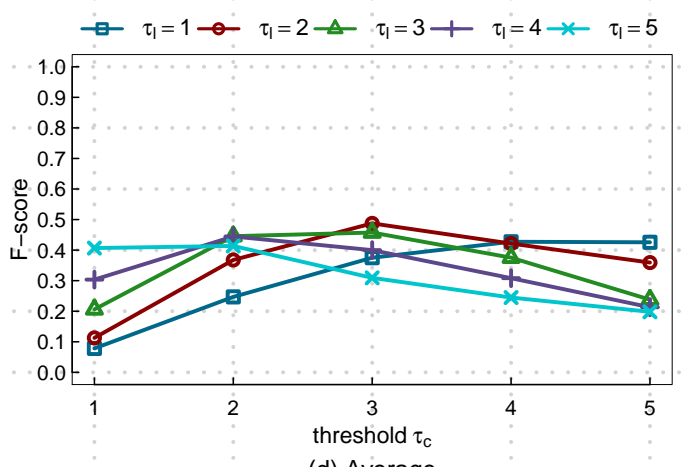

(d) Average

Figure 4.10: F-score results for the EWMA-MAD approach with the Gavagai dataset

Table 4.10: Optimal parameter sets for the EWMA-MAD approach with the Gavagai dataset

\begin{tabular}{|l|c|c|c|c|}
\hline Sentiment & Parameters & Precision & Recall & F-score \\
\hline Positive & $\tau_{c}=3, \tau_{l}=2$ & 0.720 & 0.423 & 0.533 \\
\hline Frequency & $\tau_{c}=3, \tau_{l}=3$ & 0.675 & 0.694 & 0.684 \\
\hline Negative & $\tau_{c}=2, \tau_{l}=4$ & 0.608 & 0.225 & 0.329 \\
\hline Average & $\tau_{c}=3, \tau_{l}=2$ & 0.558 & 0.504 & 0.487 \\
\hline
\end{tabular}

As shown in Figure 4.10, the F-score for the EWMA-MAD approach generally increases with increase in $\tau_{c}$ and $\tau_{l}$, until values of 2,3 , and 4 for each of these parameters were achieved. As shown in Table 4.10, the highest F-score achieved was for the neutral sentiment with the parameter set $\tau_{c}=3$ and $\tau_{l}=3$. However, this approach produced the highest average F-score for parameter set $\tau_{c}=3$ and $\tau_{l}=2$. 


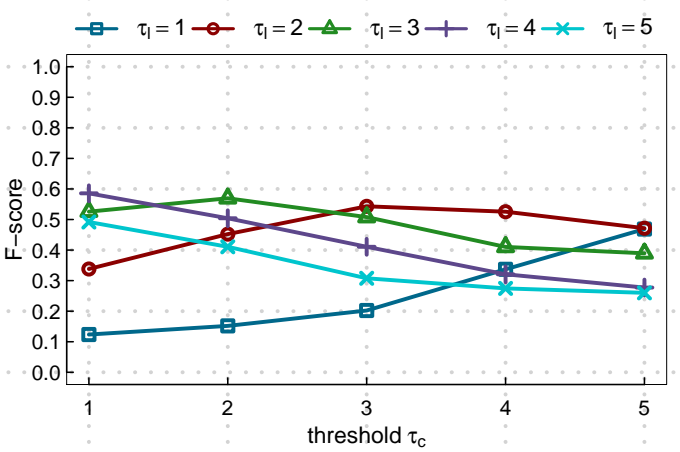

(a) Positive sentiment

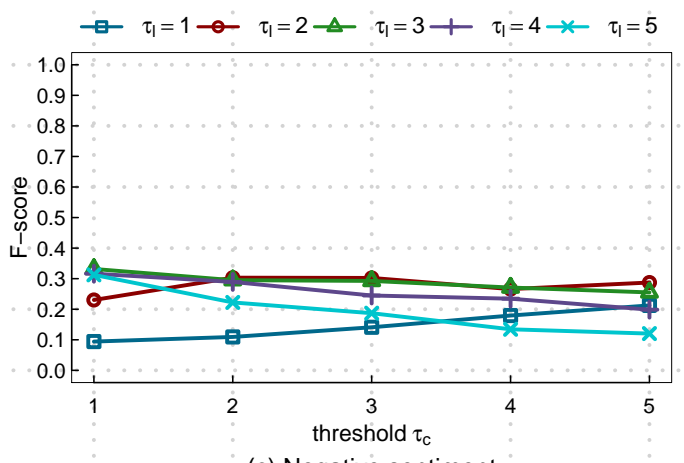

(c) Negative sentiment

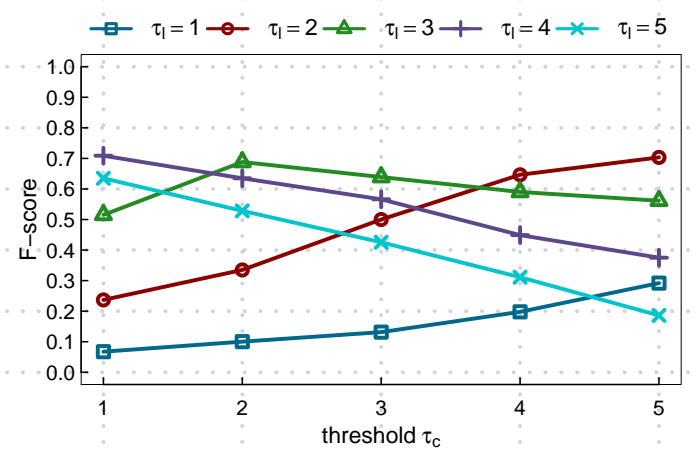

(b) Frequency

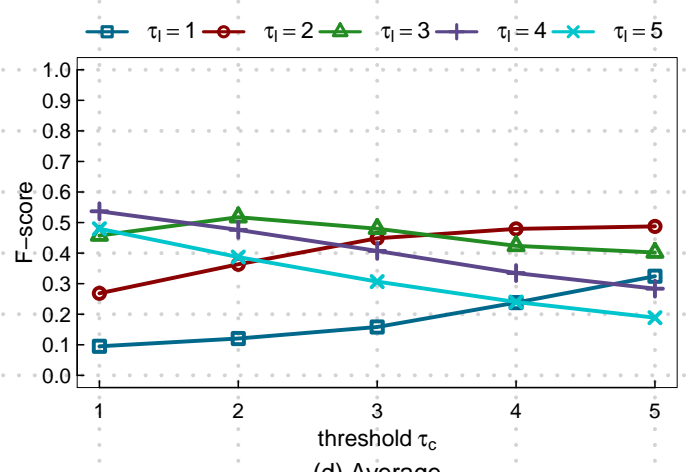

(d) Average

Figure 4.11: F-score results for the PEWMA-STD approach with the Gavagai dataset

Table 4.11: Optimal parameter sets for the PEWMA-STD approach with the Gavagai dataset

\begin{tabular}{|l|c|c|c|c|}
\hline Sentiment & Parameters & Precision & Recall & F-score \\
\hline Positive & $\tau_{c}=1, \tau_{l}=4$ & 0.745 & 0.482 & 0.585 \\
\hline Frequency & $\tau_{c}=1, \tau_{l}=4$ & 0.651 & 0.777 & 0.708 \\
\hline Negative & $\tau_{c}=1, \tau_{l}=3$ & 0.387 & 0.290 & 0.331 \\
\hline Average & $\tau_{c}=1, \tau_{l}=4$ & 0.641 & 0.495 & 0.536 \\
\hline
\end{tabular}

As shown in Figure 4.11, the F-score for the PEWMA-STD approach decreases with increases in $\tau_{c}$, only for specific values of $\tau_{l}=\{3,4,5\}$. For $\tau_{l}=\{1,2\}$ the F-score increases with increase in $\tau_{c}$. As shown in Table 4.11, for this approach the highest F-score achieved was for the neutral sentiment with parameter set $\tau_{c}=1$ and $\tau_{l}=4$. These parameter set also resulted the highest average F-score. 


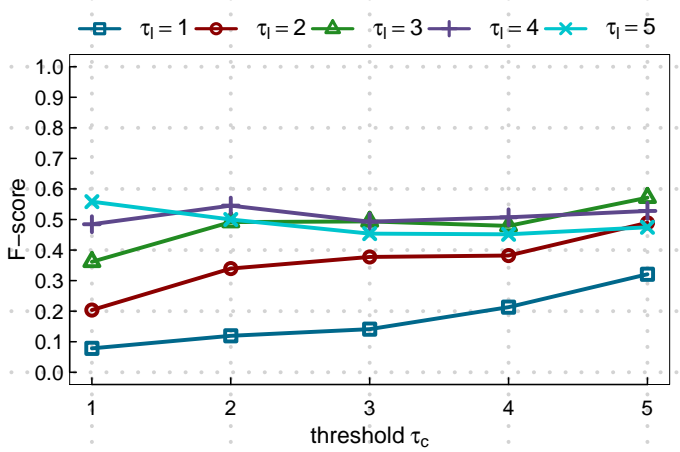

(a) Positive sentiment

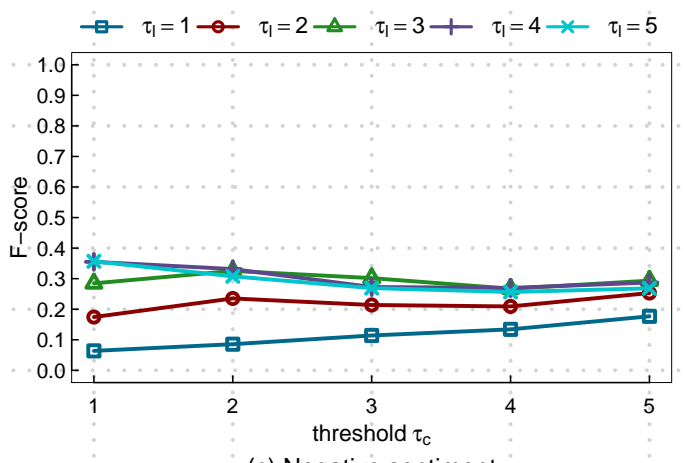

(c) Negative sentiment

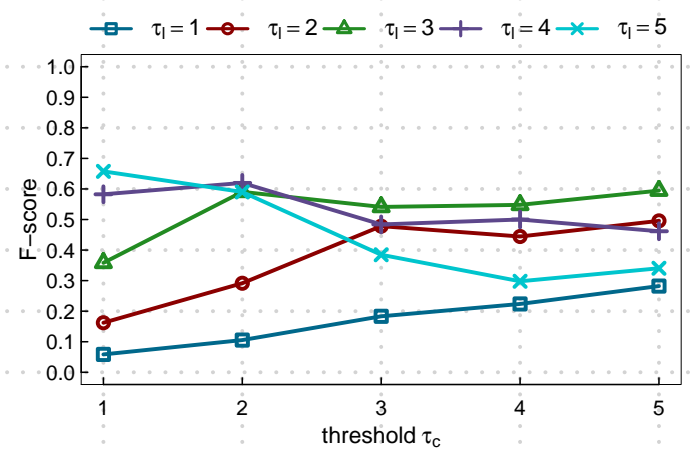

(b) Frequency

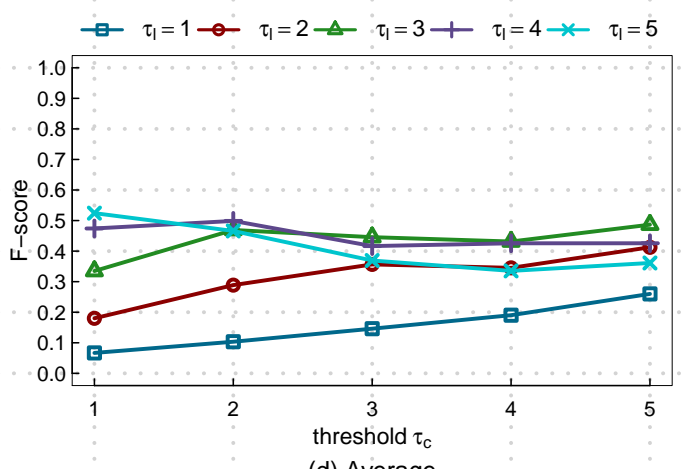

(d) Average

Figure 4.12: F-score results for the PEWMA-MAD approach with the Gavagai dataset

Table 4.12: Optimal parameter sets for the PEWMA-MAD approach with the Gavagai dataset

\begin{tabular}{|l|c|c|c|c|}
\hline Sentiment & Parameters & Precision & Recall & F-score \\
\hline Positive & $\tau_{c}=5, \tau_{l}=3$ & 0.727 & 0.470 & 0.571 \\
\hline Frequency & $\tau_{c}=1, \tau_{l}=5$ & 0.648 & 0.666 & 0.657 \\
\hline Negative & $\tau_{c}=1, \tau_{l}=5$ & 0.540 & 0.266 & 0.356 \\
\hline Average & $\tau_{c}=1, \tau_{l}=5$ & 0.604 & 0.479 & 0.524 \\
\hline
\end{tabular}

As shown in Figure 4.12, the F-score for the PEWMA-MAD approach increases with an increase in $\tau_{l}$ and decreases with $\tau_{c}$ for lower values of $\tau_{l}$ and increases with higher values of $\tau_{l}$. As shown in Table 4.12, the highest F-score achieved was for neutral sentiment with parameter setting $\tau_{c}=1, \tau_{l}=5$. This parameter setting also resulted highest average $\mathrm{F}$-score. 
The following discussion is given in order to identify the best variant approach for the TRAD technique in the context of Gavagai dataset. First, the comparative study of the behavior of the EWMA and PEWMA in relation to the parameter $\tau_{c}$ is presented. Considering the results for the EWMA approach in the context of the candidate anomaly detection stage (i.e., EWMA-STD (Figure 4.9) and EWMA-MAD (Figure 4.10)), one can see that there exists first, a direct relation $\left(\tau_{l}=\{1,2\}\right)$ and than an inverse relation $\left(\tau_{l}=\{3,4,5\}\right)$ between the F-score and the parameter $\tau_{c}$. For $\tau_{l}=\{1,2\}$, the direct relation was present, because for the small value of $\tau_{c}$ the precision was high (those that met this criteria were clearly anomalies) and the recall was adversely affected with many actual anomalies not being detected, whereas for the large value of $\tau_{c}$ the precision and recall both increased. For $\tau_{l}=\{3,4,5\}$, as the $\tau_{c}$ parameter were increased the method for determining if a data point is considered a candidate anomaly became more strict resulting in a decrease in F-score.

The results for the approaches that use PEWMA for the candidate anomaly detection stage (i.e., PEWMA-STD (Figure 4.11) and PEWMA-MAD (Figure 4.12)), show that the relation between the F-score and parameter $\tau_{c}$, in case of the PEWMA-STD approach there was a first direct relation $\left(\tau_{l}=\{1,2\}\right)$ and then an inverse relation $\left(\tau_{l}=\{3,4,5\}\right)$, whereas for the PEWMA-MAD approach there was a direct relation for all the values of $\tau_{l}$. The direct relation was present because for the small values of $\tau_{c}$ the precision was high (those that met this criteria were clearly anomalies) and the recall was adversely affected with many actual anomalies not being detected, whereas for the large value of $\tau_{c}$ the precision and recall both increased.

Comparing the performance of the EWMA and PEWMA, as discussed in Section 3.2.2, EWMA was affected by the extreme anomalies. This resulted in significant distortion in the mean value from its central tendency, making it difficult to discover additional anomalies in the local context as the threshold value increases. Because 
this dataset does not contain frequent extreme data points (low level of volatility), the performance of the EWMA and PEWMA cannot be differentiated based on their ability to adapt with the extreme value data point. Instead when the EWMA and PEWMA were compared based on F-score, it was observed that the approaches that use the PEWMA for candidate anomaly detection achieved the highest average Fscores (i.e., PEWMA-STD and PEWMA-MAD). Thus, as compared to the EWMA, the PEWMA was able to detect the true candidate anomalies at higher rate and for this dataset it was inferred that the PEWMA is better than the EWMA.

Second, in the context of the PEWMA the comparative study of the behaviour of the STD and MAD is presented in relation to the parameter $\tau_{l}$. Looking at the result of the PEWMA-STD approach (Figure 4.11), it shows that there was an inverse relation between the $\mathrm{F}$-score and specific values of $\tau_{l}=\{3,4,5\}$. Here the STD is only calculated over the data points present in the sliding window. As the number of data points were comparatively small, the results shows that the STD was extremely sensitive to the change in $\tau_{l}$ value. As the parameter $\tau_{l}$ was increased, the method for determining if a candidate anomaly is considered a legitimate anomaly become more strict, resulting in a decrease in the F-score. While this resulted in high precision (those that met this criteria were clearly anomalies), the recall was adversely affected with many actual anomalies not being detected.

The results for the PEWMA-MAD approach (Figure 4.6) shows that there was a direct relation between the F-score and parameter $\tau_{l}$. MAD is also calculated over the data points present in the sliding window. Even though the number of data points were small, the results shows that the MAD was not particularly sensitive to the change in $\tau_{l}$ value. This is because, as discussed in Section 3.2.2, the MAD is immune to the size of data, especially when the size is small it is able to represent the central tendency. 
Table 4.13: Average F-score results summarized for all the four variants of TRAD technique with the Gavagai dataset (maximum values are bold)

\begin{tabular}{|l|c|c|c|c|}
\hline Sentiment & $\begin{array}{c}\text { EWMA- } \\
\text { STD }\end{array}$ & $\begin{array}{c}\text { EWMA- } \\
\text { MAD }\end{array}$ & $\begin{array}{c}\text { PEWMA- } \\
\text { STD }\end{array}$ & $\begin{array}{c}\text { PEWMA- } \\
\text { MAD }\end{array}$ \\
\hline Positive & 0.561 & 0.533 & $\mathbf{0 . 5 8 5}$ & 0.571 \\
\hline Neutral & $\mathbf{0 . 7 1 6}$ & 0.684 & 0.708 & 0.657 \\
\hline Negative & 0.300 & 0.329 & 0.331 & $\mathbf{0 . 3 5 6}$ \\
\hline Average & 0.520 & 0.487 & 0.536 & $\mathbf{0 . 5 2 4}$ \\
\hline
\end{tabular}

Comparing the performance of the STD and MAD, as discussed in Section 3.2.2 and Section 3.2.1, within the sliding window, there are comparatively fewer data points. If these data points have extreme values, then the simple average that is used to calculate STD fails to represent the central tendency of the window. Considering MAD, the median that is used to calculate MAD was able to represent the mean close to its central tendency for the window. Thus, from these results it is inferred that MAD is able to detect the true legitimate anomalies with higher accuracy as compared to the STD.

Table 4.13 summarizes the results from all the four approaches for the TRAD technique. It can be seen that when considering the average F-scores, the PEWMA-STD approach performed better than others achieving the value of $0.536\left(\tau_{c}=1, \tau_{l}=4\right)$ in the average comparison, which is slightly ahead from the F-score of $0.524\left(\tau_{c}=\right.$ $\left.1, \tau_{l}=5\right)$ for the PEWMA-MAD approach. While the other approaches under the TRAD technique approached close to this value for certain parameter sets, given the resilience of PEWMA-MAD and PEWMA-STD to the threshold parameters (specifically for $\tau_{l}>3$ ), it is concluded that in the context of the Gavagai dataset, these are the superior approaches.

Simple Linear Regression Approach: The range of values for the parameters $n$ and $\tau$ were same as chosen for the Le Tour de France dataset, except the window size parameter $k$. The optimal value for $k$ was independently evealuated and it was set to 
21 days for this dataset. The results of the evaluation conducted for identifying the optimal window size is given in the Appendix Table B.2. The results are presented in Figure 4.13. An optimal parameter setting for each of the three sentiment classes and the average of them is presented in Table 4.14.

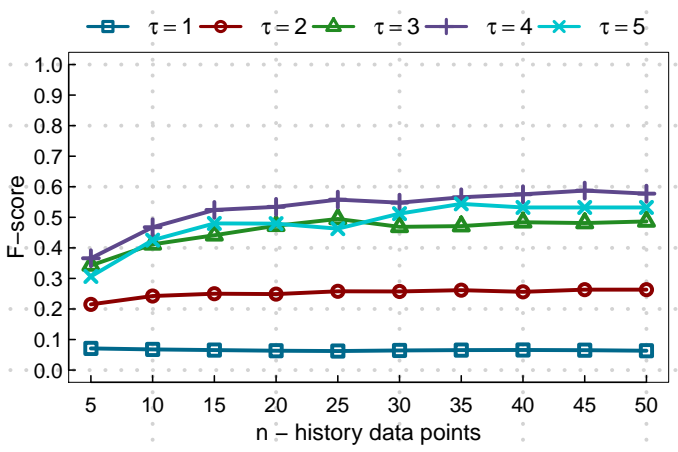

(a) Positive sentiment

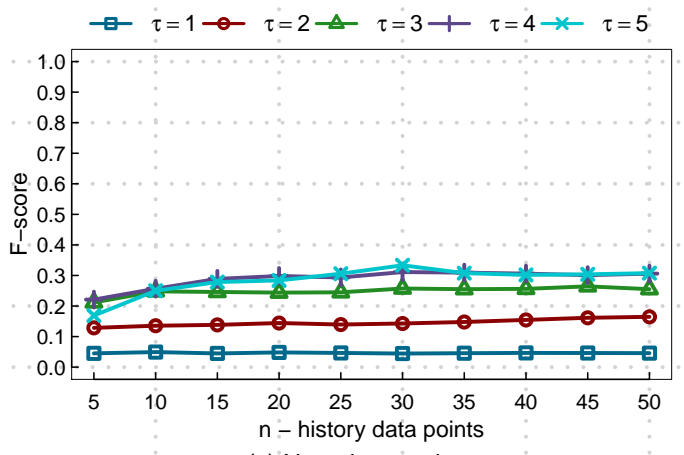

(c) Negative sentiment

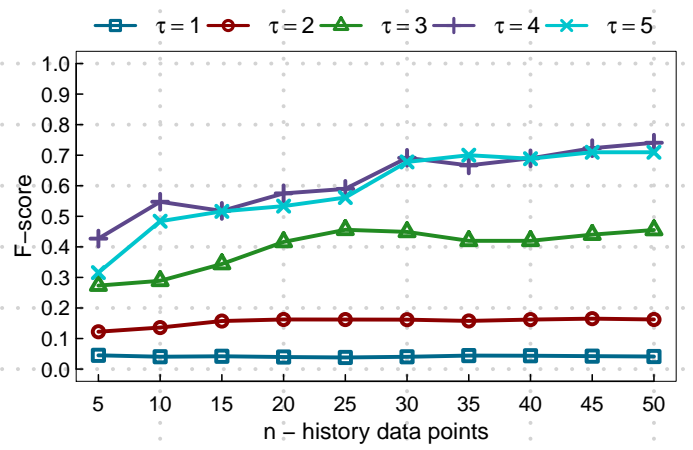

(b) Frequency

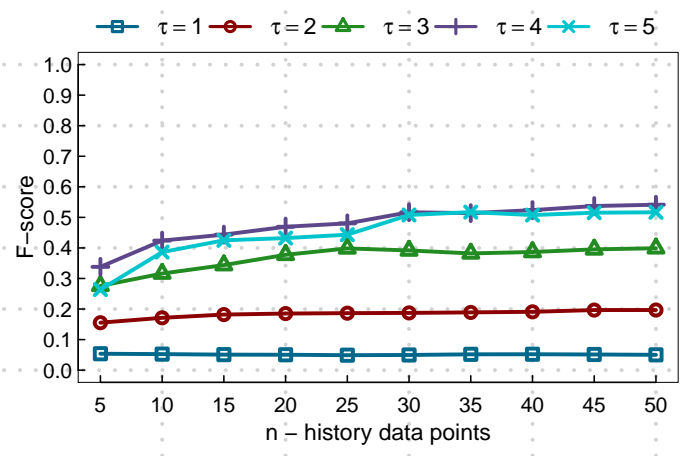

(d) Average

Figure 4.13: F-score results for the SLR approach with the Gavagai dataset

Table 4.14: Optimal parameter sets for the SLR approach with the Gavagai dataset

\begin{tabular}{|l|c|c|c|c|}
\hline Sentiment & Parameters & Precision & Recall & F-score \\
\hline Positive & $n=45, \tau=4$ & 0.724 & 0.494 & 0.587 \\
\hline Frequency & $n=50, \tau=5$ & 0.846 & 0.611 & 0.709 \\
\hline Negative & $n=30, \tau=5$ & 0.812 & 0.209 & 0.333 \\
\hline Average & $n=50, \tau=4$ & 0.620 & 0.513 & 0.541 \\
\hline
\end{tabular}

Looking at the results for the SLR technique in Figure 4.13, when considering the parameter $n$, there is a gradual increase in the F-score with the increase in the 
value of $n$. The SLR technique is based on predictive analysis and performs well for detecting rare extreme peaks. This dataset does consist of rare extreme peaks and the anomalies are generally distant from each other as compared to the Le Tour de France dataset. When compared with the PEWMA-MAD and PEWMA-STD approaches in Figure 4.15, the SLR approach performed marginally better with an average F-score of $0.541(n=50, \tau=4)$.

Local Outlier Factor Approach: The range of values for the LOF technique parameters $k$, and $\tau$ were same as chosen for the TDF dataset. The optimal value for the number of neighbours parameter $N$ were also chosen same as for the TDF dataset.

Looking at the results for the local outlier factor technique in Figure 4.14, it shows that the F-score remains constant as the value of $\tau$ and $k$ increases. As shown in Table 4.15 , for this approach the highest F-score achoieved was for the frequency with the parameter setting $k=40, \tau=5$. When averaging the results, the highest $\mathrm{F}$-score was for the parameter set $k=40, \tau=5$.

However, when the result of the LOF technique is compared with the SLR technique in Table 4.16 the performance of the LOF technique is lower with the F-score of 0.473 , whereas the SLR technique achieved 0.541. Moreover, comparing the LOF to the PEWMA-STD and PEWMA-MAD approach, the former approach performed poorly.

Discussion: From Table 4.16 it is clear that for the Gavagai's dataset, the PEWMASTD, PEWMA-MAD, and SLR approaches achieved similarly high F-scores (0.536, 0.524 , and 0.541, respectively) across all the other candidate methods. While the PEWMA-STD and PEWMA-MAD techniques approached close to the maximum achieved value by a short margin, the SLR technique is the superior approach when considered for the Gavagai's dataset. 


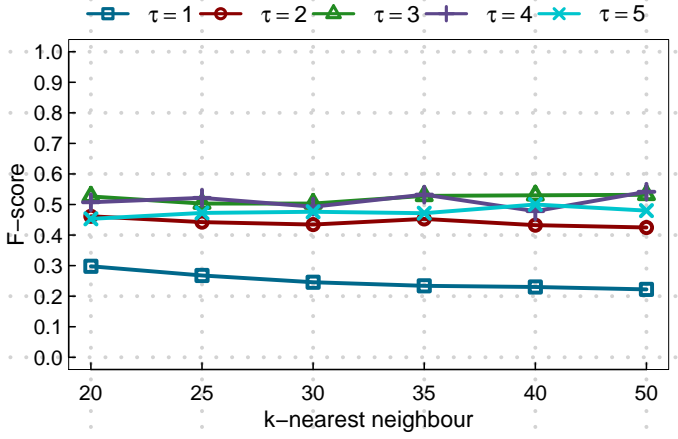

(a) Positive sentiment

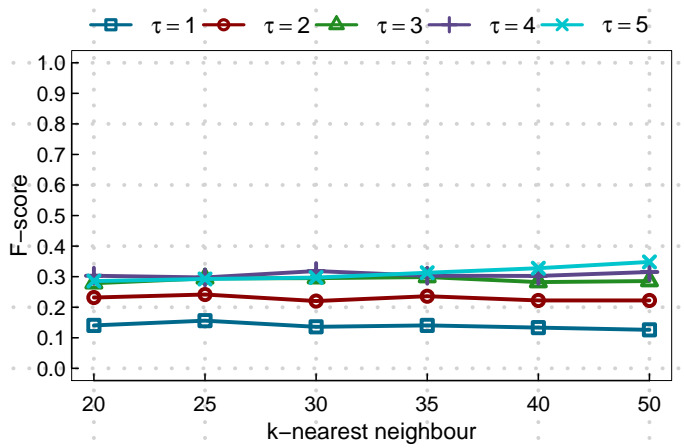

(c) Negative sentiment

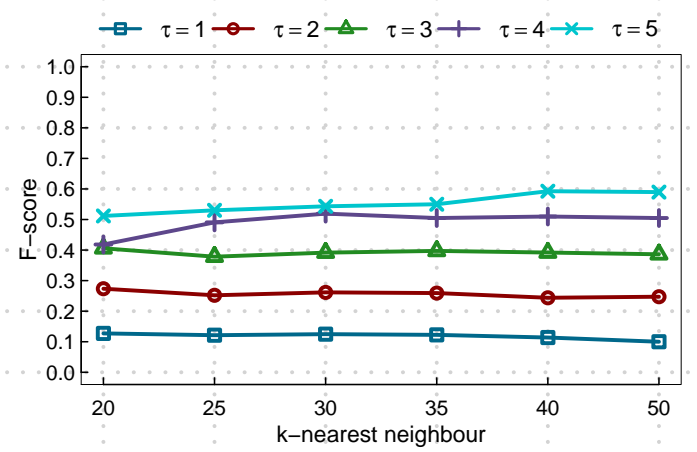

(b) Frequency

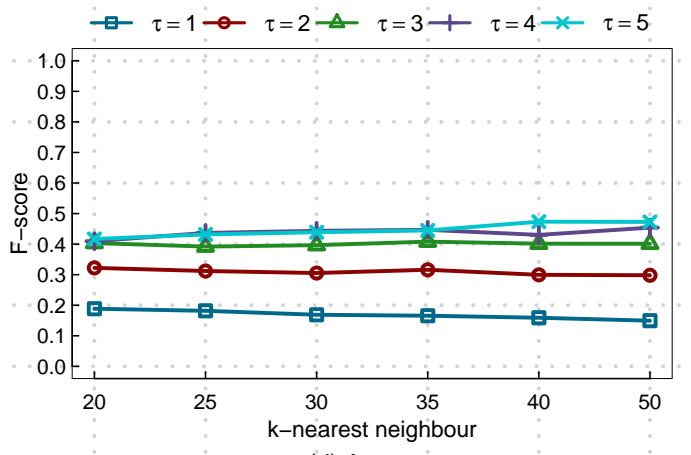

(d) Average

Figure 4.14: F-score results for the LOF approach with the Gavagai dataset

Table 4.15: Optimal parameter sets for the LOF approach with the Gavagai dataset

\begin{tabular}{|l|c|c|c|c|}
\hline Sentiment & Parameters & Precision & Recall & F-score \\
\hline Positive & $k=50, \tau=4$ & 0.661 & 0.458 & 0.541 \\
\hline Frequency & $k=40, \tau=5$ & 0.533 & 0.666 & 0.592 \\
\hline Negative & $k=50, \tau=5$ & 0.574 & 0.250 & 0.348 \\
\hline Average & $k=40, \tau=5$ & 0.625 & 0.421 & 0.473 \\
\hline
\end{tabular}

Table 4.16: Average F-score results summarized for PEWMA-STD, PEWMA-MAD, SLR, and LOF methods with the Gavagai dataset

\begin{tabular}{|c|c|c|c|c|}
\hline Sentiment & $\begin{array}{c}\text { PEWMA- } \\
\text { STD }\end{array}$ & $\begin{array}{c}\text { PEWMA- } \\
\text { MAD }\end{array}$ & SLR & LOF \\
\hline Positive & 0.585 & 0.571 & $\mathbf{0 . 5 8 7}$ & 0.541 \\
\hline Frequency & 0.708 & 0.657 & $\mathbf{0 . 7 0 9}$ & 0.592 \\
\hline Negative & 0.331 & $\mathbf{0 . 3 5 6}$ & 0.333 & 0.348 \\
\hline Average & $\mathbf{0 . 5 3 6}$ & 0.524 & 0.541 & 0.473 \\
\hline
\end{tabular}




\subsubsection{Summary of Results}

To summarize the results from the experiments conducted in this Thesis, the performance of the top three techniques are compared in the context of both the datasets (TDF 2013 and Gavagai datasets) as illustrated in the Figure 4.15. The two techniques that have performed consistently well in both the datasets are PEWMAMAD and PEWMA-STD. However, the third member for the TDF 2013 dataset was EWMA-MAD and the first member (but not by much) for the Gavagai dataset was SLR.

First, considering the TDF 2013 dataset which has high level of volatility, the PEWMA-MAD approach is the top performer. This is because of the PEWMA's

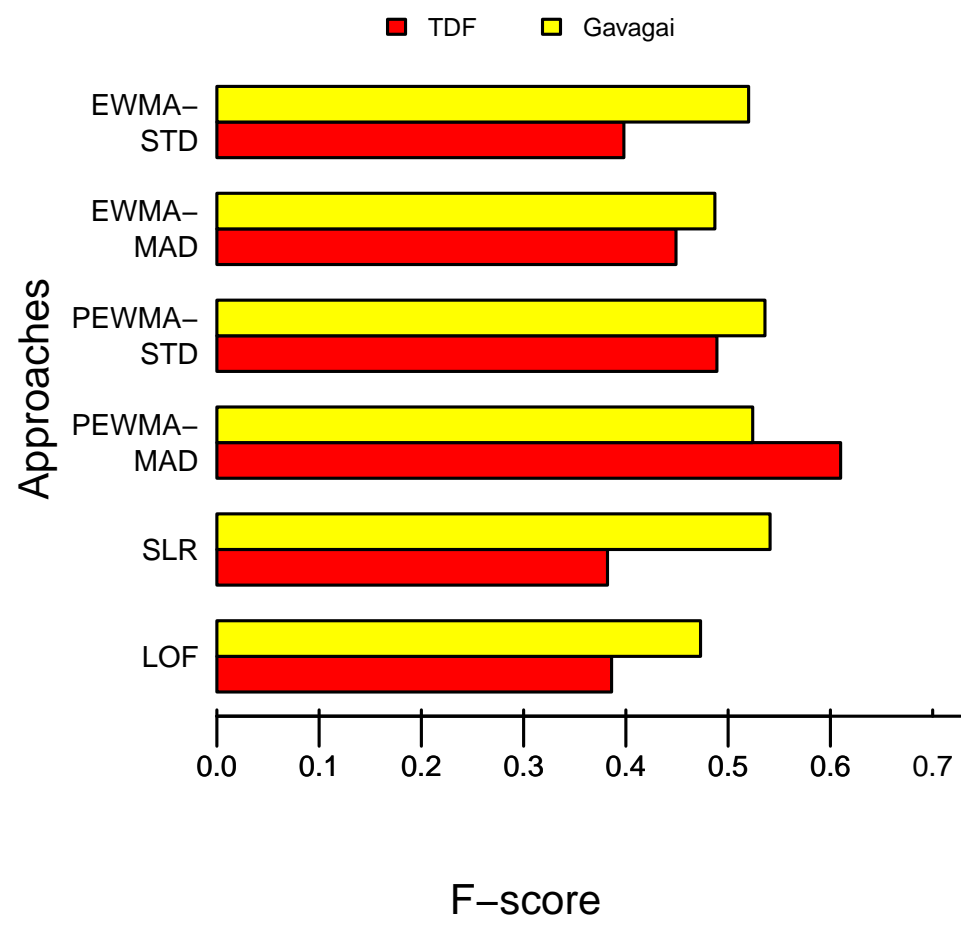

Figure 4.15: Results summary providing the ranking of the techniques for each datasets, based on the highest average F-score 
ability, as discussed in Section 3.2.2, to dynamically adjust the decay factor in order to be resilient against the extreme values. With such an ability, the result is that the mean value which is used to calculate the candidate anomaly score was not distorted and the accuracy in detecting the true anomalies was increased. This reasoning also stands true for the median and MAD approach while calculating the legitimate anomaly score. MAD is also resilient against the extreme values, especially when the dataset is comparatively small (see Section 3.2.2).

Considering the Gavagai dataset, which has relatively low volatility, the SLR technique is the top performer (average F-score of 0.541). To understand this result, recall the theory for the SLR technique as discussed in Section 2.2.4. The SLR technique predicts a forthcoming data point with a linear model that looks at the historical data points, and compares it to the actual data point. The prediction error between the predicted and actual data points are measured. If the error value is statistically large enough from a threshold, it is labelled as anomaly. The behaviour of the SLR technique with both the datasets is shown in Figure 4.16. In case of the Gavagai dataset, it has a low level of volatility and thus does not contain statistically extreme values. Because of this, the prediction error does not deviate significantly from the mean and this allows the SLR technique to detect the true anomalies in the Gavagai dataset. In contrast the TDF 2013 dataset has a high level of volatility and thus contains statistically extreme values. Because of this, when the extreme value data points are predicted it results in large prediction error. Further, the distorting effect of this error in the mean value is carried forward and neutralized gradually over time, which causes the subsequent anomalies being missed and resulting in low recall.

The last item to note from the Figure 4.15 is that the two approaches that specifically use the PEWMA for the candidate anomaly detection stage in the TRAD technique are consistently present in the top three performer list for both the datasets. 


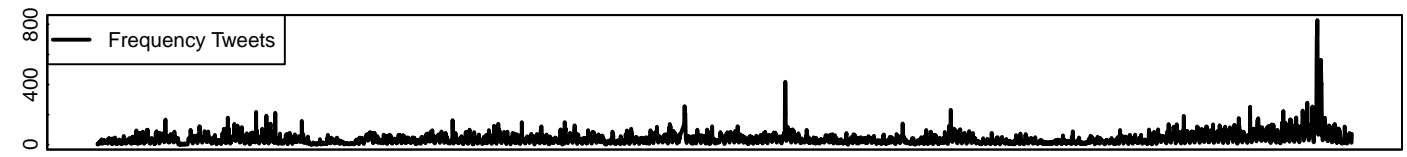

(a) Frequency, Gavagai dataset

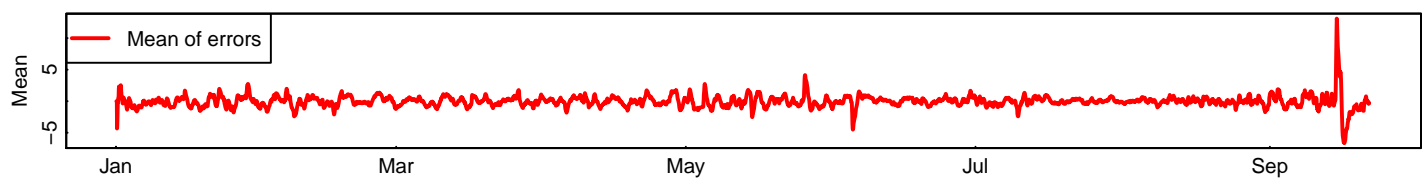

(b) Frequency, Gavagai dataset

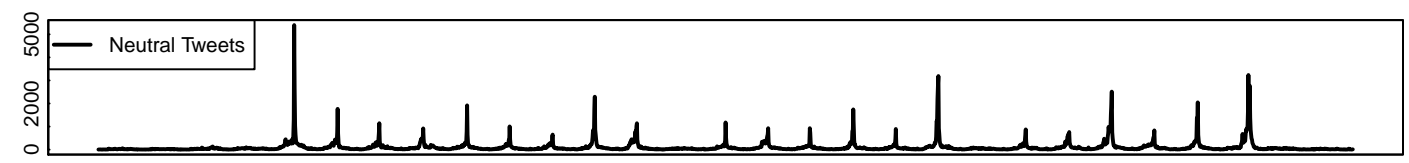

(c) Neutral Sentiment, TDF 2013 dataset

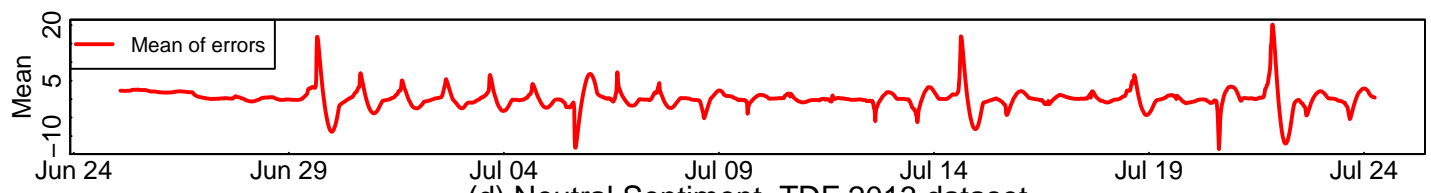

(d) Neutral Sentiment, TDF 2013 dataset

Figure 4.16: Result demonstrating the performance of the SLR technique with Gavagai (Window $=10$ days, $n=50, \tau=4$ ) and $\mathrm{TDF} 2013$ (Window $=6$ days, $n=50, \tau=4)$ datasets.

These are the PEWMA-STD technique for the Gavagai dataset which is the second best technique and performed slightly better than PEWMA-MAD. This result indicate that STD performs better than MAD for estimating the deviation within the sliding window when the number of data points are small with low volatility nature.

Finally, the results of the experimental evaluation performed in this Chapter are summarized. The LOF technique was one of poorest performers in both the datasets and thus its results were unacceptable for the task of anomaly detection in usergenerated data streams. In the case of the SLR technique, it was at the sixth place (worst performer) for the TDF 2013 dataset, while for the Gavagai dataset it was the top performer. The SLR technique is highly sensitive to the extreme values which makes it a less suitable technique for data streams having high volatility. The PEWMA-MAD technique was the top performer for the TDF 2013 dataset, while for 
the Gavagai dataset it was in third place, but with a short margin. The PEWMAMAD technique consistently performed well with the data streams that had statistically low as well as high level of volatility, making it a good choice when one does not know what to expect from the data (as in the case with user-generated content that is dependent on events and people's opinions on these). 


\section{Chapter 5}

\section{Conclusions}

In this chapter, a section is devoted to a summary of the contributions, and an overview of the limitation and future work. In the first section, the contributions and results of the research in this thesis are presented. In the second section, limitations and suggestions for continuing the development of the ideas presented in this thesis are given.

\subsection{Contributions}

The research presented in this thesis is oriented towards the development of a realtime approach to automatically detect sentiment-based anomalies (RSAD) in Twitter data streams. Detecting anomalies in data streams is challenging because of the constraints on space and time utilization. On Twitter, the popularity of topics change over time and brief periods of high popularity are reflected in the sentiment time series as sudden peaks. The presence of repetitive peaks makes it challenging to detect the true anomalies in the data stream. The proposed two-stage real-time anomaly detection (TRAD) technique addresses this problem by augmenting a sliding window based approach with two stage anomaly detection. The experimental evaluation shows that this technique, and in particular the PEWMA-MAD variation, can effectively 
tolerate the repetitiveness in the data and detects the true anomalies with acceptable accuracy. This approach is practical to implement, robust against concept drift, and scalable to handle data streams with varying velocity. To our knowledge, a real-time approach specifically to detect the sentiment-based anomalies in the user-generated content has not been previously presented in the literature.

The sentiment anomalies for different topics are different in nature. The adaptive characteristic of the TRAD technique enables it to be applicable to detect sentiment anomalies related to the topics from a wide range of application domains. Examples of the application domains are presented below. Detecting sentiment anomalies in tweets related to corporate companies (such as \#Nestle or \#Google) can assist the stock holders to predict changes in the stock for that company, federal election (such as \#CanadianElection or \#USelections) can uncover changes in public sentiment towards the political parties or candidates, sport events (such as \#TDF or \#Roughriders) can allow the communications manager to detect anomalous reactions of fans during an ongoing game, catastrophic weather events (such as \#Earthquake or \#Hurricane) can help to detect changes in sentiment in order to estimate the actual disaster based on the negative tweets separate from the positive tweets that show support to the region, and specific technologies (such as \#SelfDrivingCar or \#Windows10) can help to understand what are the immediate reactions of people when such next generation technology is announced or released.

The following are the major contributions of the research conducted in this thesis, corresponding to the set of goals listed in Section 1.2:

1. Introduced a definition for sentiment-based anomaly (Section 3.1), which allows us to formulate the problem of independently detecting the rare anomalies in each class of sentiment on Twitter. In order to identify a rare anomaly, two types of anomalies were introduced, candidate anomaly and legitimate anomaly. 
A candidate anomaly represents an anomaly in the local context and has the potential to become a rare anomaly. A legitimate anomaly represents an rare anomaly with respect to a sliding window of a given length. A legitimate anomaly in an individual sentiment class on Twitter is referred to as a sentiment-based anomaly.

2. A two stage real-time anomaly detection algorithm called TRAD was proposed (Section 3.2.2).

(a) In order to detect the sentiment-based anomalies within a fixed amount of storage, a sliding window based approach was used. The computational complexity of the method used in the TRAD algorithm is linear because of the incremental nature of the computation. Thus, the TRAD algorithm satisfies the memory consumption and run time complexity constraints sufficiently well to be considered real-time.

(b) The EWMA and PEWMA incremental moving average techniques were used in order to handle the temporal concept drift.

3. The real-time sentiment-based anomaly detection (RSAD) technique was implemented using the TRAD algorithm (Section 3.5) in the context of Twitter.

(a) A real-time stream processing framework that consists of two consecutive steps was implemented within Apache Storm. The Twitter stream is first divided into multiple parallel streams using a classifier, and then the desired data processing (e.g., anomaly detection) is performed on each stream independently.

(b) The RSAD technique was implemented within this framework, using a sentiment classifier to divide the stream into three sentiment classes.

(c) The anomaly detection step implements the TRAD algorithm, which is executed on independent threads for each class of sentiment. 
(d) The multi-threading capability of Storm framework was used for classifying the Twitter data stream and for concurrently executing the TRAD algorithm with respect to multiple queries.

(e) The multi-threading capability of Storm framework was also used for preprocessing the Twitter data stream and for concurrently executing the TRAD algorithm with respect to more than one query.

4. An empirical evaluation of the TRAD algorithm was performed using two datasets. Four variants of the algorithm were compared with each other and then with two alternative baseline techniques: linear regression and local outlier factor (Chapter 4$)$.

\subsection{Limitations and Future Work}

The research presented in this thesis has a number of limitations and leaves future research opportunities that may lead to improved sentiment-based anomaly detection. In this section, the limitations of the proposed approach and potential research that could be performed to overcome these limitations are presented.

Sentiment classifier: The overall performance of the RSAD technique with respect to accurately identifying sentiment-based anomalies in a Twitter data stream depends on the accuracy of the sentiment classifier used to classify the tweets. In this work, a sentiment classifier named Sentiment 140 [42] was used. Although this service was specifically designed for classifying tweets, it has a few limitations. First, the classifier does not consider the actual domain relevant to the tweet (such as sports, politics, or technology). Second, the classifier considers only three classes of sentiment (positive,

neutral, and negative), and strictly classifies a tweet into one of the three classes. However, additional classes of sentiment could be defined such as tension, depression, 
anger, vigour, fatigue, and confusion [9]. Third, because it is a third party service, the sentiment classifier could not be trained according to the specific needs of the research. In future work, a sentiment analysis method could be adopted that addresses the aforementioned limitations of Sentiment $140[9,10,28]$ and performs sentiment classification in an incremental manner over a data stream. Furthermore, the sentiment classifier itself can be replaced with another classifier that can operate on the variables available in the tweet object. For example, a spatial clustering algorithm can be used to classify the tweet objects based on their geo-location variables.

Extended definition of sentiment anomaly: In this thesis, sentiment-based anomaly is defined with respect to the sudden increase in tweets related to a topic. This definition represents a change in the frequency of individual sentiment classes. However, in some cases, a sudden increase in an individual sentiment class may not be a true sentiment-based anomaly if it occurs at the same time as a similar increase in other sentiment classes. The relation between multiple sentiment time series can be tracked with correlation analysis. In future work, a method could be devised to facilitate the detection of changes over time in the correlation between the sentiment classes to exclude such patterns.

Approach to identify seasonal anomalies: Seasonality in a time series is a regular pattern of changes that repeats over fixed time periods. For example, consider a sentiment time series for a city (e.g., all the tweets with hashtag \#cityofregina). Since people tweet less on weekends than weekdays, a seasonality factor could be used to describe the regular reduction in the number of tweets that occur on the weekends in comparison to the other days. Such seasonal patterns can be modelled as normal behaviour in the sentiment time series, while any change from the expected seasonal pattern will be considered to be a seasonal sentiment anomaly. Kejariwal et al.[51] proposed an offline piecewise median-based approach to detect these seasonal 
anomalies in the context of Twitter. Although their approach showed promising results, it cannot be readily applied to Twitter data streams because of its limitation to offline analysis. To enhance the approach described in this thesis, an online method could be devised to model the seasonality in the Twitter data streams.

Data distribution model: The TRAD approach presented in this thesis assumes that the data stream is generated from a fixed normal distribution with a mean and a standard deviation. However, because of the dynamic behaviour of data streams the underlying distribution that generates the data stream can change over time. Such a fixed data distribution may not be adequate to capture the dynamic behaviour of data streams. In future work, first, different data distributions which are known to be robust for small data sets can be considered, such as long tail distribution, tdistribution, or Poisson distribution [3]. Second, instead of any particular fixed data distribution function, a data distribution function that can incrementally adapted to the changes in a data stream, such as an incremental Gaussian mixture model [44] or an adaptive kernel density estimator [40], can be considered.

Multivariate anomaly detection: A tweet object in a Twitter data stream is associated with a rich set of variables including time, text, geolocation, retweet count, and follower count. In the TRAD approach, the only variables considered when calculating the anomaly score are the text (sentiment) and the time. However, the anomaly score could be altered to account for additional factors that might allow true sentiment anomalies to be detected more accurately. In future work, variables such as location, retweet count, and follower count could be evaluated with respect to their effect on the anomaly score, in order to identify other factors influencing sentiment anomalies.

Experiments with datasets from other domains: In this thesis, the datasets used for the evaluation were from the sport and political domains. The efficacy of the 
TRAD approach should be tested with datasets from other domains such as Twitter data streams related to a specific corporation, a consumer product, or a natural disaster.

Experiments with single stage techniques: The alternative baseline techniques considered for the comparative evaluations were optimized to execute in two stages, for a fair comparison with the proposed two-stage anomaly detection technique. However, in future work the baseline techniques could be executed in a single stage to isolate the value of two-stage approach used in this work. 


\section{References}

[1] Estimating the volatility of financial time series. http://fedc.wiwi. hu-berlin.de/xplore/tutorials/xfghtmlnode107.html. (accessed October $10,2015)$.

[2] Charu C. Aggarwal. An introduction to data streams. In Charu C. Aggarwal, editor, Data Streams: Models and Algorithms, Advances in Database Systems, pages 1-8. Springer, 2007.

[3] Charu C. Aggarwal. Linear models for outlier detection. In Charu C. Aggarwal, editor, Outlier Analysis, pages 75-99. Springer, 2013.

[4] Apache Storm. https://storm.apache.org/. (accessed January 1, 2015).

[5] Marco Avvenuti, Stefano Cresci, Andrea Marchetti, Carlo Meletti, and Maurizio Tesconi. EARS (earthquake alert and report system): A real time decision support system for earthquake crisis management. In Proceedings of the International Conference on Knowledge Discovery and Data Mining, pages 1749-1758, 2014 .

[6] Vic Barnett and Lewis Toby. Outliers in Statistical Data. Wiley Series in Probability and Mathematical Statistics. John Wiley \& Sons Inc, New York, third edition, 1994. 
[7] Albert Bifet. Adaptive learning and mining for data streams and frequent patterns. SIGKDD Explorations Newsletter, 11(1):55-56, 2009.

[8] Albert Bifet and Eibe Frank. Sentiment knowledge discovery in Twitter streaming data. In Proceedings of the International Conference on Discovery Science, pages $1-15,2010$.

[9] Johan Bollen, Alberto Pepe, and Huina Mao. Modeling public mood and emotion: Twitter sentiment and socio-economic phenomena. Computing Research Repository, http://arxiv.org/abs/0911.1583, 2009.

[10] Henri Bouma, Olga Rajadell, Daniül Worm, Corné Versloot, and Harry Wedemeijer. On the early detection of threats in the real world based on open-source information on the internet. In Proceedings of the International Conference on Information Technologies and Security, pages 15-16, 2012.

[11] Markus M. Breunig, Hans-Peter Kriegel, Raymond T. Ng, and Jörg Sander. LOF: Identifying density-based local outliers. In Proceedings of the SIGMOD International Conference on Management of Data, pages 93-104, 2000.

[12] Dariusz Brzeziński. Mining data streams with concept drifts. Master's thesis, Poznan University of Technology, 2010. Available from http://www.cs.put.poznan.pl/dbrzezinski/publications/ConceptDrift.pdf.

[13] Kevin M. Carter and William W. Streilein. Probabilistic reasoning for streaming anomaly detection. In Proceedings of the Workshop on Statistical Signal Processing, pages 377-380, 2012.

[14] Varun Chandola, Arindam Banerjee, and Vipin Kumar. Anomaly detection: A survey. ACM Computing Surveys, 41(3):10-13, 2009. 
[15] Denis Cousineau and Sylvain Chartier. Outliers detection and treatment: A review. International Journal of Psychological Research, 3(1):58-67, 2010.

[16] Aron Culotta. Towards detecting influenza epidemics by analyzing Twitter messages. In Proceedings of the First Workshop on Social Media Analytics, pages $115-122,2010$.

[17] Jeffrey Dean and Sanjay Ghemawat. MapReduce: Simplified data processing on large clusters. Communication of the ACM, 51(1):107-113, 2008.

[18] Nicholas A. Diakopoulos and David A. Shamma. Characterizing debate performance via aggregated Twitter sentiment. In Proceedings of the SIGCHI Conference on Human Factors in Computing Systems, pages 1195-1198, 2010.

[19] Angiulli Fabrizio and Fassetti Fabio. Detecting distance-based outliers in streams of data. In Proceedings of the International Conference on Information and Knowledge Management, pages 811-820, 2007.

[20] Wei Fan and Albert Bifet. Mining big data: Current status, and forecast to the future. SIGKDD Exploration Newsletter, 14(2):1-5, 2013.

[21] Atefeh Farzindar and Khreich Wael. A survey of techniques for event detection in Twitter. Journal of Computational Intelligence, 31(1):132-164, 2015.

[22] Tony Finch. Incremental calculation of weighted mean and variance. University of Cambridge Computing Service, 4:11-5, 2009.

[23] Manish Gupta, Jing Gao, Charu C. Aggarwal, and Jiawei Han. Outlier detection for temporal data: A survey. IEEE Transactions on Knowledge and Data Engineering, 26(9):2250-2267, 2014. 
[24] Jheser Guzman and Barbara Poblete. On-line relevant anomaly detection in the Twitter stream: An efficient bursty keyword detection model. In Proceedings of the Workshop on Outlier Detection and Description, pages 31-39, 2013.

[25] David J. Hill and Barbara S. Minsker. Anomaly detection in streaming environmental sensor data: A data-driven modeling approach. Journal on Environmental Modelling \& Software, 25(9):1014-1022, 2010.

[26] Orland Hoeber, Larena Hoeber, Maha El Meseery, Kenneth Odoh, and Radhika Gopi. Visual Twitter analytics (Vista): Temporally changing sentiment and the discovery of emergent themes within sport event tweets. 40(1):25-41, 2015.

[27] Orland Hoeber, Larena Hoeber, Laura Wood, Ryan Snelgrove, Isabella Hugel, and Dayne Wagner. Visual Twitter analytics: Exploring fan and organizer sentiment during Le Tour de France. In Proceedings of the VIS Workshop on Sports Data Visualization, pages 1-7, 2013.

[28] Yuheng Hu, Fei Wang, and Subbarao Kambhampati. Listening to the crowd: Automated analysis of events via aggregated Twitter sentiment. In Proceedings of the International Joint Conference on Artificial Intelligence, pages 2640-2646, 2013.

[29] Yexi Jiang, Chunqiu Zeng, Jian Xu, and Tao Li. Real time contextual collective anomaly detection over multiple data streams. In Proceedings of the SIGKDD Workshop on Outlier Detection 85 Description under Data Diversity, pages 2330, 2014 .

[30] Daniel Keim, Gennady Andrienko, Jean-Daniel Fekete, Carsten Görg, Jörn Kohlhammer, and Guy Melançon. Visual analytics: Definition, process, and challenges. In Andreas Kerren, John T. Stasko, Jean-Daniel Fekete, and Chris 
North, editors, Information Visualization: Human-Centered Issues and Perspectives, volume 4950, pages 154-175. Springer, 2008.

[31] Daniel A. Keim, Florian Mansmann, and Jim Thomas. Visual analytics: How much visualization and how much analytics? SIGKDD Explorer Newsletter, pages 5-8, 2010.

[32] Shvachko Konstantin, Hairong Kuang, Sanjay Radia, and Robert Chansler. The Hadoop distributed file system. In Proceedings of the Symposium on Mass Storage Systems and Technologies, pages 1-10, 2010.

[33] Christophe Leys, Olivier Klein, Philippe Bernard, and Laurent Licata. Detecting outliers: Do not use standard deviation around the mean, use absolute deviation around the median. Journal of Experimental Social Psychology, 49(4):764-766, 2013.

[34] Adam Marcus, Michael S. Bernstein, Osama Badar, David R. Karger, Samuel Madden, and Robert C. Miller. TwitInfo: Aggregating and visualizing microblogs for event exploration. In Proceedings of the SIGCHI Conference on Human Factors in Computing Systems, pages 227-236, 2011.

[35] David Minnen, Charles Isbell, Irfan Essa, and Thad Starner. Detecting subdimensional motifs: An efficient algorithm for generalized multivariate pattern discovery. In IEEE International Conference on Data Mining, pages 601-606, 2007.

[36] Gerhard Münz and Georg Carle. Application of forecasting techniques and control charts for traffic anomaly detection. In Proceedings of the ITC Specialist Seminar on Network Usage and Traffic, 2008. 
[37] Shanmugavelayutham Muthukrishnan. Data Streams: Algorithms and Applications. Now Publishers Inc, 2005.

[38] Zizi Papacharissi and Maria de Fatima Oliveira. Affective news and networked publics: The rhythms of news storytelling on \#egypt. Journal of Communication, 62(2):266-282, 2012.

[39] Dragoljub Pokrajac, Aleksandar Lazarevic, and Longin Jan Latecki. Incremental local outlier detection for data streams. In Proceedings of the IEEE Symposium on Computational Intelligence and Data Mining, pages 504-515, 2007.

[40] Shiblee Sadik and Le Gruenwald. Online outlier detection for data streams. In Proceedings of the ACM Symposium on International Database Engineering 8 Applications, pages 88-96, 2011.

[41] Shiblee Sadik and Le Gruenwald. Research issues in outlier detection for data streams. SIGKDD Explorer Newsletter, 15(1):33-40, 2014.

[42] Sentiment 140. http://www.sentiment140.com/. (accessed December 10, 2014).

[43] Songwon Seo. A review and comparison of methods for detecting outliers in univariate data sets. Master's thesis, University of Pittsburgh, 2006. Available from http://d-scholarship.pitt.edu/7948/1/Seo.pdf.

[44] Mingzhou Song and Hongbin Wang. Highly efficient incremental estimation of Gaussian mixture models for online data stream clustering. In Proceedings of the conference on Intelligent Computing: Theory and Applications III, pages 174-183, 2005. 
[45] Yutaka Tanaka and Yuichi Mori. Principal component analysis based on a subset of variables: variable selection and sensitivity analysis. American Journal of Mathematical and Management Sciences, 17(2):61-89, 1997.

[46] Mike Thelwall, Kevan Buckley, and Georgios Paltoglou. Sentiment in Twitter events. Journal of the American Society for Information Science and Technology, 62(2):406-418, 2011.

[47] Ankit Toshniwal, Siddarth Taneja, Amit Shukla, Karthik Ramasamy, Jignesh M. Patel, Sanjeev Kulkarni, Jason Jackson, Krishna Gade, Maosong Fu, Jake Donham, Nikunj Bhagat, Sailesh Mittal, and Dmitriy Ryaboy. Storm@ Twitter. In Proceedings of the SIGMOD International Conference on Management of Data, pages 147-156, 2014.

[48] Andranik Tumasjan, Timm O. Sprenger, Philipp G. Sandner, and Isabell M. Welpe. Predicting elections with Twitter: What 140 characters reveal about political sentiment. In Proceedings of the International AAAI Conference on Weblogs and Social Media, pages 178-185, 2010.

[49] Twitter Java Library. http://twitter4j.org/en/index.html. (accessed December 10, 2014).

[50] Twitter Public Streams. https://dev.twitter.com/streaming/public. (accessed December 10, 2014).

[51] Owen Vallis, Jordan Hochenbaum, and Arun Kejariwal. A novel technique for long-term anomaly detection in the cloud. In Proceedings of the USENIX Conference on Hot Topics in Cloud Computing, pages 1-15, 2014.

[52] Andreas Yacob and Olof Nilsson. Non-parametric anomaly detection in sentiment 
time series data. Master's thesis, Uppsala University, 2015. Available from http://uu.diva-portal.org/smash/get/diva2:807192/FULLTEXT01.pdf.

[53] Kenji Yamanishi and Jun-ichi Takeuchi. A unifying framework for detecting outliers and change points from time series. IEEE Transactions on Knowledge and Data Engineering, 18(4):482-492, 2006.

[54] Kenji Yamanishi, Jun-ichi Takeuchi, Graham Williams, and Peter Milne. On-line unsupervised outlier detection using finite mixtures with discounting learning algorithms. Journal of Data Mining and Knowledge Discovery, 8(3):275-300, 2004.

[55] Matei Zaharia, Mosharaf Chowdhury, Tathagata Das, Ankur Dave, Justin Ma, Murphy McCauley, Michael J. Franklin, Scott Shenker, and Ion Stoica. Resilient distributed datasets: A fault-tolerant abstraction for in-memory cluster computing. In Proceedings of the USENIX Conference on Networked Systems Design and Implementation, pages 1-2, 2012.

[56] Wang Zhaoxia, Victor Joo, Chuan Tong, Xin Xin, and Hoong Chor Chin. Anomaly detection through enhanced sentiment analysis on social media data. In Proceedings of the International Conference on Cloud Computing Technology and Science, pages 917-922, 2014. 


\section{Appendix A}

\section{Detailed Results for TDF 2013 dataset}

\section{A.1 Evaluation for Window Size in TDF 2013}

The Tables A.1-A.3 presents the results of evaluation conducted with TDF 2013 dataset to determine the optimal value among the individual list of predefined values for the window size parameter $(n)$ in TRAD approach, window size parameter in SLR approach, number of neighbours parameters in LOF approach. The optimal value for a parameter is the one for which the highest average F-score was obtained. For each value in the list of predefined values, the algorithm was executed and the F-score results were recorded for each of the sentiment classes as well as the average of them.

The window size parameter value having the highest average F-score was chosen to conduct further evaluations for each of the techniques. 
Table A.1: Results for the optimal window size parameter $(n)$ for the TRAD approach with the TDF dataset

\begin{tabular}{|c|c|c|c|c|}
\hline Window Size (Days) & Frequency & Positive & Negative & Average \\
\hline 1 & 12.08 & 8.26 & 6.29 & 8.88 \\
\hline 3 & 13.69 & 9.25 & 6.76 & 9.90 \\
\hline $\mathbf{6}$ & $\mathbf{1 4 . 1 2}$ & 9.80 & 7.16 & $\mathbf{1 0 . 3 6}$ \\
\hline 7 & 13.95 & 9.77 & 7.17 & 10.30 \\
\hline 10 & 13.84 & 9.73 & 7.20 & 10.26 \\
\hline 15 & 13.70 & 9.78 & 7.17 & 10.22 \\
\hline 20 & 13.82 & $\mathbf{9 . 9 0}$ & 7.23 & 10.32 \\
\hline 30 & 13.71 & 9.84 & 7.19 & 10.25 \\
\hline 40 & 13.71 & 9.84 & $\mathbf{7 . 3 1}$ & 10.25 \\
\hline
\end{tabular}

Table A.2: Results for the optimal window size parameter $(k)$ for the SLR approach with the TDF dataset

\begin{tabular}{|c|c|c|c|c|}
\hline Window Size (Days) & Positive & Neutral & Negative & Average \\
\hline 1 & 13.71 & 9.84 & 7.19 & 10.25 \\
\hline 3 & $\mathbf{1 4 . 1 2}$ & 9.86 & 7.10 & 10.36 \\
\hline $\mathbf{6}$ & 12.49 & $\mathbf{1 7 . 7 0}$ & $\mathbf{8 . 3 2}$ & $\mathbf{1 2 . 8 3}$ \\
\hline 7 & 12.18 & 16.70 & 7.73 & 12.20 \\
\hline 10 & 12.05 & 16.97 & 7.96 & 12.33 \\
\hline 15 & 11.88 & 16.16 & 7.06 & 11.70 \\
\hline 20 & 11.97 & 16.33 & 7.06 & 11.78 \\
\hline 30 & 16.50 & 12.07 & 7.13 & 11.90 \\
\hline 40 & 16.53 & 12.05 & 7.13 & 11.90 \\
\hline
\end{tabular}

Table A.3: Results for the optimal window size parameter $(N)$ for the LOF approach with the TDF dataset

\begin{tabular}{|c|c|c|c|c|}
\hline \# Neighbours (Data points) & Positive & Frequency & Negative & Average \\
\hline 40 & 6.24 & 10.80 & 4.39 & 7.14 \\
\hline 45 & 7.02 & 11.94 & 4.84 & 7.93 \\
\hline 50 & 7.40 & 13.03 & 5.11 & 8.51 \\
\hline 60 & 8.65 & 14.38 & 5.38 & 9.47 \\
\hline $\mathbf{7 0}$ & $\mathbf{8 . 8 2}$ & $\mathbf{1 4 . 8 5}$ & $\mathbf{5 . 8 9}$ & $\mathbf{9 . 8 5}$ \\
\hline
\end{tabular}




\section{Appendix B}

\section{Detailed Results for Gavagai dataset}

\section{B.1 Evaluation for Window Size in Gavagai dataset}

The Tables B.1-B.2 presents the results of evaluation conducted with the Gavagai dataset to determine the optimal value among the individual list of predefined values for the window size parameter $(n)$ in TRAD approach, window size parameter in SLR approach. For the Gavagai dataset the window size evaluation result for LOF approach is not presented as the results were similar to the TDF 2013 dataset. The optimal value for a parameter is the one for which the highest average F-score was obtained. For each value in the list of predefined values, the algorithm was executed and the F-score results were recorded for each of the sentiment classes as well as the average of them. The window size parameter value having the highest average F-score was chosen to conduct further evaluations for each of the techniques. 
Table B.1: Results for the optimal window size parameter $(n)$ for the TRAD approach with the Gavagai dataset

\begin{tabular}{|c|c|c|c|c|}
\hline Window Size (Days) & Positive & Frequency & Negative & Average \\
\hline 1 & 6.52 & 4.70 & 3.44 & 4.89 \\
\hline 3 & 9.34 & 7.77 & 5.31 & 7.47 \\
\hline 6 & 9.68 & 9.64 & 5.61 & 8.31 \\
\hline 7 & 9.78 & 9.69 & 5.35 & 8.27 \\
\hline 10 & 9.67 & 10.27 & 5.71 & 8.55 \\
\hline 15 & 9.72 & $\mathbf{1 0 . 8 9}$ & 5.48 & 8.69 \\
\hline $\mathbf{2 0}$ & 9.86 & 11.02 & 5.83 & 8.90 \\
\hline 30 & 9.92 & 10.82 & 6.07 & 8.94 \\
\hline 40 & $\mathbf{1 0 . 0 3}$ & 10.60 & $\mathbf{6 . 2 4}$ & $\mathbf{8 . 9 5}$ \\
\hline
\end{tabular}

Table B.2: Results for the optimal window size parameter $(k)$ for the SLR approach with the Gavagai dataset

\begin{tabular}{|c|c|c|c|c|}
\hline Window Length (Days) & Positive & Frequency & Negative & Average \\
\hline 1 & 14.95 & 12.59 & 8.05 & 11.86 \\
\hline 3 & 17.66 & 15.89 & 9.76 & 14.44 \\
\hline 6 & $\mathbf{1 8 . 2 3}$ & 17.72 & 10.00 & 15.32 \\
\hline 7 & 17.88 & 17.76 & 9.90 & 15.18 \\
\hline 10 & 17.94 & 17.58 & $\mathbf{1 0 . 3 0}$ & 15.27 \\
\hline 15 & 18.04 & 17.84 & 9.97 & 15.28 \\
\hline $\mathbf{2 1}$ & 17.83 & $\mathbf{1 7 . 9 9}$ & 10.13 & $\mathbf{1 5 . 3 2}$ \\
\hline 30 & 18.04 & 16.72 & 10.35 & 15.04 \\
\hline 40 & 18.03 & 16.68 & 10.67 & 15.13 \\
\hline
\end{tabular}

